\title{
Thyroid tumors -
}

\section{A modern diagnostic approach improves}

\section{the management of thyroid cancer patients}

\section{Eva Sigstad}

Department of Pathology

The Norwegian Radium Hospital

Oslo University Hospital

Faculty of Medicine

University of Oslo

Oslo, 2012

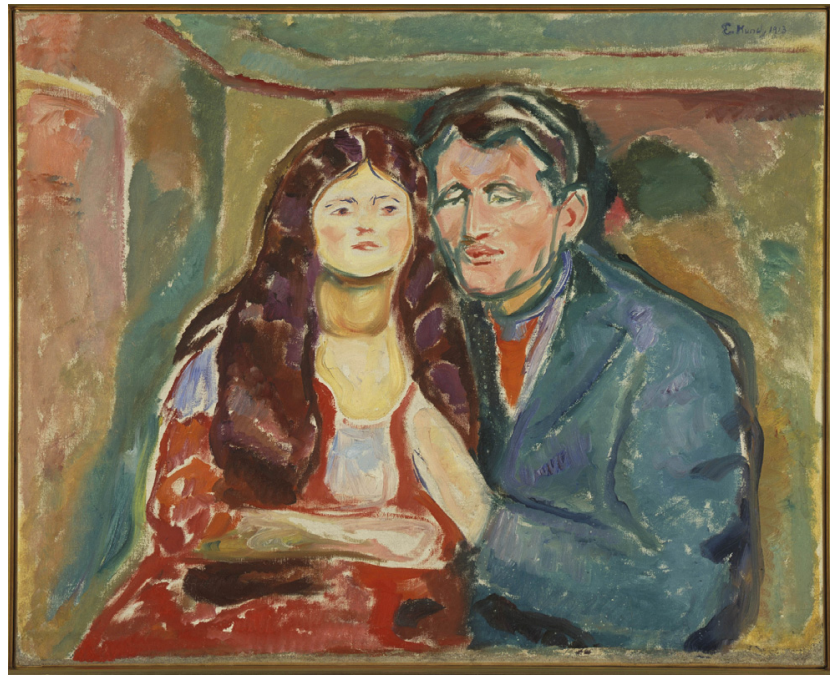

[M 55]

Photo: (C) Munch Museum

Edvard Munch: The Seducer 1913

Oil on canvas

$80.5 \times 100 \mathrm{~cm}$

Munch Museum, Oslo.

Art piece:

(C) Munch Museum / The Munch-Ellingsen Group / BONO, Oslo 2011 
(C) Eva Sigstad, 2012

Series of dissertations submitted to the Faculty of Medicine, University of Oslo No. 1314

ISBN 978-82-8264-344-3

All rights reserved. No part of this publication may be reproduced or transmitted, in any form or by any means, without permission.

Cover: Inger Sandved Anfinsen.

Printed in Norway: AIT Oslo AS.

Produced in co-operation with Unipub.

The thesis is produced by Unipub merely in connection with the thesis defence. Kindly direct all inquiries regarding the thesis to the copyright holder or the unit which grants the doctorate. 
Table of Contents

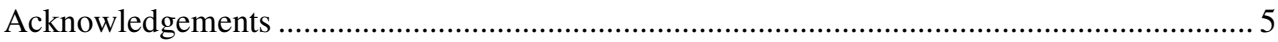

Preface

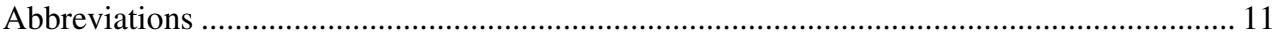

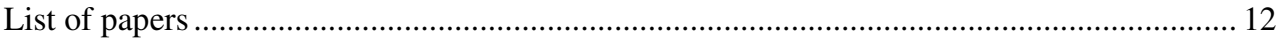

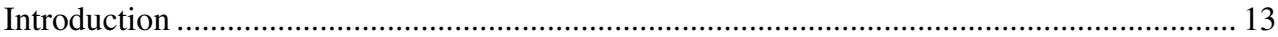

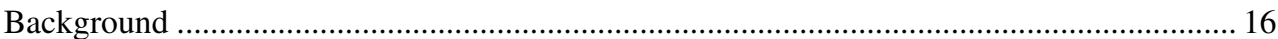

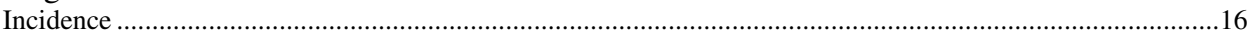

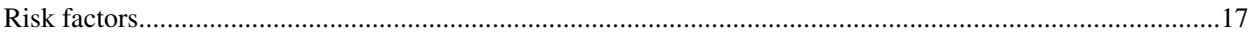

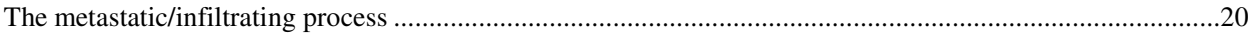

Extracellular environment and thyroid carcinogenesis/tumorigenesis .......................................................20

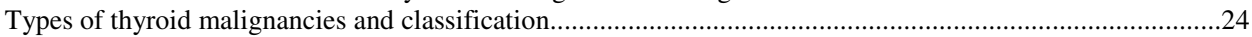

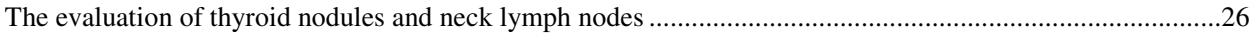

Diagnostic markers for discriminating benign and malignant tumors ...........................................................28

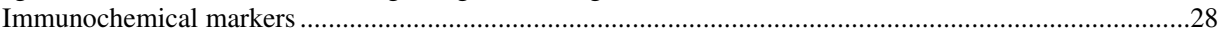

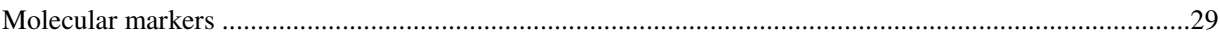

Treatment of differentiated thyroid carcinoma and metastases ....................................................................

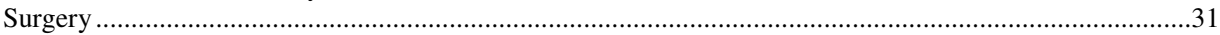

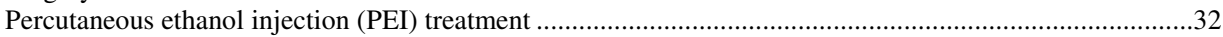

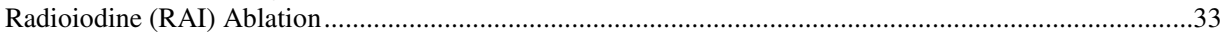

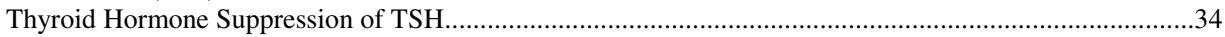

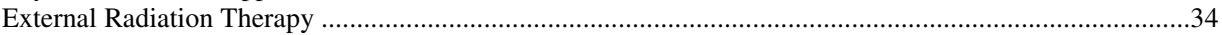

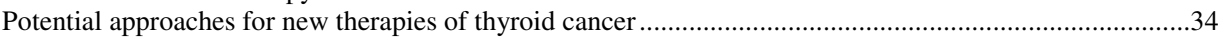

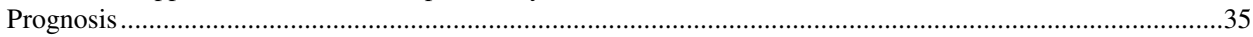

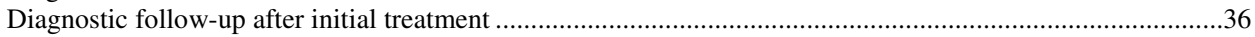

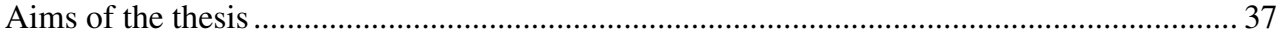

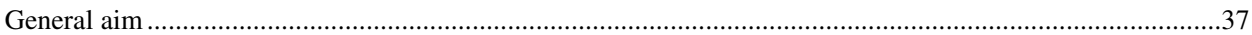

Specific aims

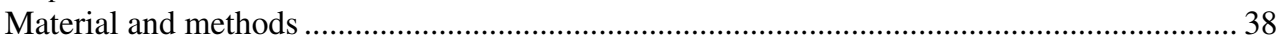

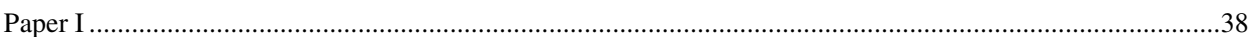

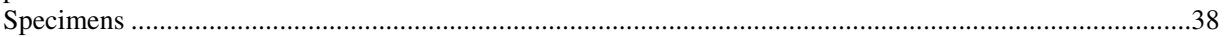

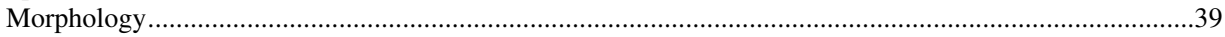

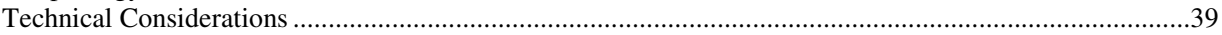

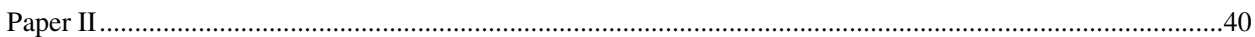

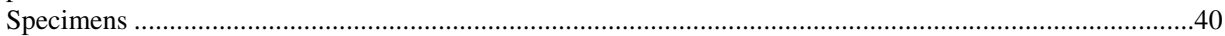

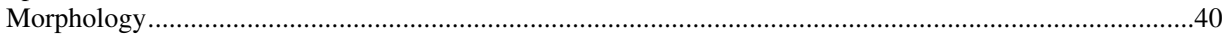

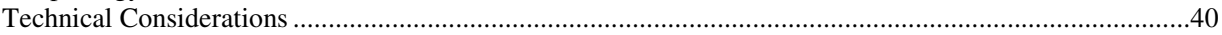

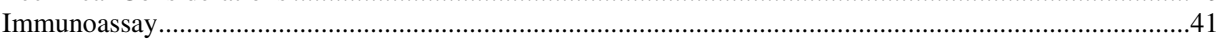

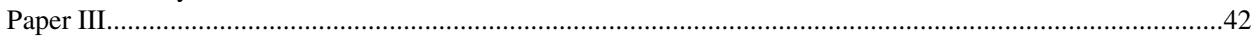

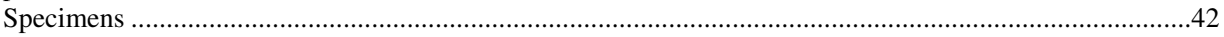

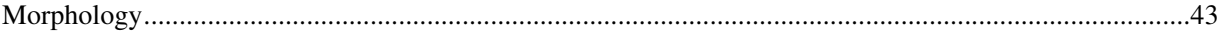

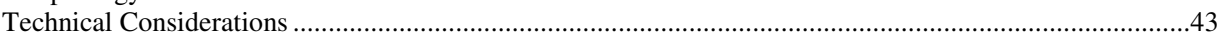

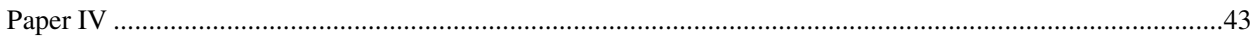

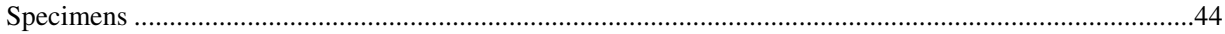

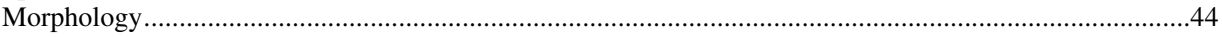

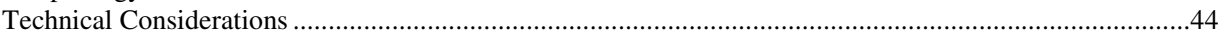

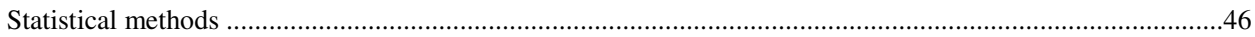

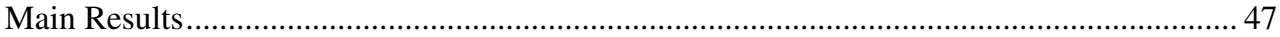

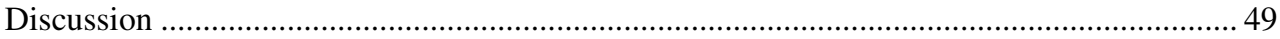

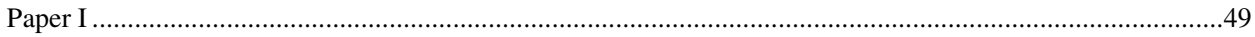

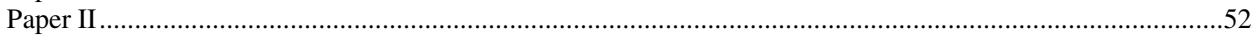

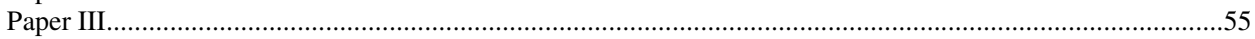

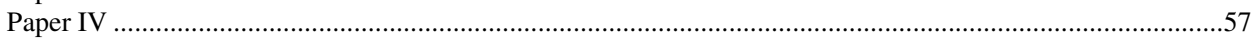

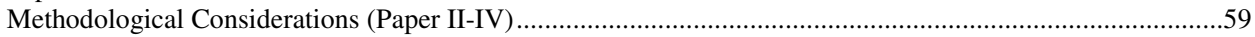

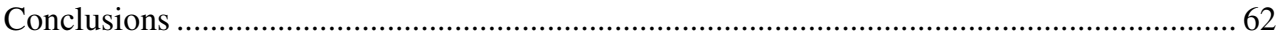

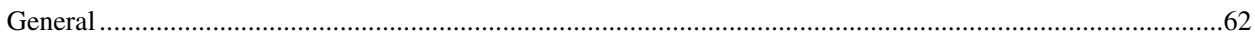


Specific.

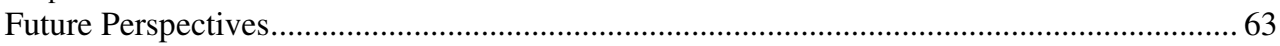

The role of the Pathologist in future management of patients with thyroid lesions ..........................................63

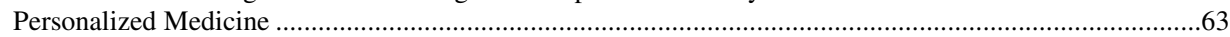

Informatics in Practical Pathology ………………………….............................................................65

The role of multidisciplinary groups in managing thyroid lesions ................................................................65

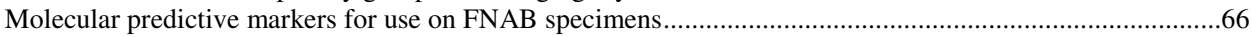

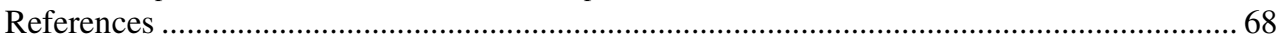

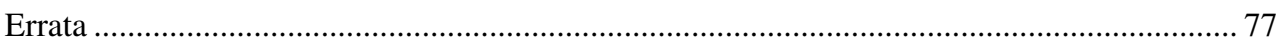

Paper I-IV 


\section{Acknowledgements}

This research work was carried out at the Department of Pathology in collaboration with the Department of Radiology and the Department of Medical Biochemistry at The Norwegian Radium Hospital, Oslo University Hospital and partly financed by grant from the Norwegian Cancer Society and South-Eastern Norway Regional Health Authority.

First of all, I wish to thank my supervisors at the Norwegian Radium Hospital:

I wish to express my deepest gratitude to my supervisor Prof. Aasmund Berner for inspiring me to go into the exciting field of thyroid pathology. Thank you for always sharing your knowledge and enthusiasm. I value the time you let me deepen in to the different aspects of thyroid management. Thank you for your daily motivation and humoristic comments, and for all your constructive suggestions for each paper and the entire thesis.

To Professor Trine Bjøro, my co-supervisor, for getting hold of grants from the Norwegian Cancer Society and South-Eastern Norway Regional Health Authority- this was enabling me to get time for working with the papers and the thesis. I gratefully appreciate the inspiration and encouragements you have provided. Thank you for giving me the opportunity to collaborate with highly skilled physicians and researchers in different departments, and for your contributions through all stages of my work.

To Professor Jahn M. Nesland, the former Head of the Department of Pathology, for always inspiring and encouraging me to carry out my scientific ideas. Your suggestions to the manuscripts and the thesis are highly valued. I warmly want to thank you for your kind and empathic support. 
To Krystyna Kotanska Grøholt at Department for Pathology for being an excellent supervisor and colleague in everyday diagnostics. You have showed me the importance of carrying out a "second look". Thank you for sharing your enormous knowledge and for spreading enthusiasm. I highly appreciate your thoughtfulness and friendship.

To Arne Heilo at Department for Radiology, for sharing your knowledge and ideas, and including me in your projects. It is a tremendous pleasure to part of the unique collaboration at the Ultrasound lab.

To Elisabeth Paus and David J. Warren at the Department for Medical Biochemistry for your inestimable contribution performing the immunoassays and the Western Blotting. Thank you for all your extensive and thorough work ensuring a high quality of the methods used, and for your important contribution to the preparation of manuscripts.

To Ruth Holm and Ellen Hellesylt for your great contribution performing excellent immunohistochemical staining. Thank you for always taking time for discussions and for fast and tedious execution of the staining.

I wish to thank my collaborators at Department of Pathology, Trond V. Bogsrud, Kristin Holgersen Fagerlid and Olav Inge Håskjold at Department of Radiology, and Lars H. Jørgensen at department of Thoracic Surgery.

To Professor Manuel Sobrinho-Simões for showing me the importance of considering all different aspects of modern pathologic diagnostics, and never forget to keep focus on how new diagnostic modalities always should be considered to the interest of the patients. 
To my dear sisters, Solveig and Jenny-Anne, my brothers Helge and Håkon, and all my friends for supporting me and giving me balance in life. Special thanks to Anne, Mette, Jeanne Mette, Kristina and Cybèle.

In the memory of my mother and father who always supported and encouraged me.

To my dearest Thomas and our enthusiastic son Sondre, with all my love and gratitude. Thank you for your joy, care and your patience. 


\section{Preface}

In his book Tumeurs Humaines published in French in 1956 and translated to English in 1970

(Human Tumors) (1), the French Canadian pathologist Pierre Masson made the statement:

"No classification is more difficult to establish than that of thyroid epitheliomas.... very few are adapted to a precise classification”. In 1964, In The New England Journal of Medicine, Veith and co-workers presented the paper "The Nodular Thyroid Gland and Cancer- A Practical Approach to the Problem" in which the following statements was made: "Unlike most other tumors, thyroid cancer has an unusually broad spectrum of behaviour. At one extreme is the well differentiated, slow-growing process that behaves in an almost benign manner. At the other extreme is the poorly differentiated, rapidly fatal anaplastic process. Knowledge of the broad zone between these two extremes is limited...." (2). These statements, written 60-70 years ago, are still elucidating our present situation interest. Until the 1990ties, differentiated thyroid carcinomas were principally divided in the two main categories: Follicular and Papillary. Further sub classification of thyroid tumors was of limited interest. However, during the last two decades, several publications have described different variants of thyroid neoplasias. The different morphological subtypes turn out to demonstrate distinct biologic behaviours at onset and during follow-up and additionally different overall survival. Thus, sub classification of these tumors is urgent for the clinicians, and our improved understanding of molecular mechanisms for development also allows future targeted therapy.

Most of the lesions disclosed in the thyroid gland are benign. The fact that the number of thyroid lesions examined are steadily raising due to a more extensive use of radiological devices with constantly improved resolution has become a significant challenge. Not only have the effort to find markers capable of separating benign and malignant lesions in 
preoperative specimens exploded, but the need for a multidisciplinary approach has become a prerequisite for optimal patient treatment.

As a cytopathologist and surgical pathologist with a special interest in the pathology of the thyroid gland, I have for years performed on-site evaluation of the fine needle aspirations (FNAB) as well as assessment of thyroid histological specimens. The on-site evaluation includes clinical cooperation in collecting the patient's anamnestic data and carrying out the cytological specimen. This subspecialisation as well as regular meetings in a multidisciplinary team guarantees a high quality diagnostics and treatment and have in our hospital acted as an arena for development of collaborative projects. Being a member of a multidisciplinary team for several years, I have been involved in several steps of the management of patients with thyroid lesions, including speaking to the patients to obtain valuable anamnestic information. Additionally, I have performed the FNAB, watched US-imaging and listened to the radiologist interpretation. In the last years I have also had the opportunity to integrate the interpretation of the thyroid specimens with electronically accessible information from medical reports, laboratory data, flow-cytometry, genetic studies and multiple knowledge databases. But not at least, I have had the pleasure to work closely together with dedicated physicians from a wide range of specialities and my thesis is an unreservedly product of this multidisciplinary collaboration. 


\section{Abbreviations}

ARG2: Arginase, type II

AKT: v-akt murine thymoma viral oncogene

BRAF: v-raf murine sarcoma viral oncogene homolog B1

C1orf25 (FAM129A): chromosome 1 open reading frame 24

CK19: cytokeratin 19

CLND: central lymph node dissection

DDIT3: DNA-damage-inducible transcript 3

DTC: differentiated thyroid carcinoma

ECM: extracellular matrix

EGFR: epidermal growth factor receptor

EMT: epithelial-mesenchymal transition

FAM129A (C1orf24): family with sequence similarity 129, member A

FMTC: familial medullary thyroid carcinoma

FN1: fibronectin 1

FNAB: fine-needle aspiration biopsy

Tg-FNAB: thyroglobulin in wash-out from fine needle aspiration biopsy

FTA: follicular thyroid adenoma

FTC: follicular thyroid carcinoma

GAL3: galactin 3

HBME 1: anti mesothelioma antibody 1

${ }^{131} \mathrm{I}$ : iodine 131 , radioiodine

IHC: immunohistochemistry

ITM1 (STT3A): interferon induced transmembrane protein 1

LRP1B: low-density lipoprotein receptor-related protein

MAPK: mitogen-activated protein kinase

MEN: multiple endocrine neoplasias

MTC: medullary thyroid carcinoma

PAX8/PPAR $\gamma$ : paired box 8/ peroxisome proliferator-activated receptor gamma

PTC: papillary thyroid carcinoma

PEI: percutaneous ethanol injection

PI3K: phosphoinositide-3-kinase

RAI: radioactive iodine

RAS: RAt Sarcoma

RET: ret proto-oncogene

S-Tg: serum thyroglobulin

S-TgAb: S-Tg antibodies

STT3A (ITM1): subunit of the oligosaccharyltransferase complex, homolog A

Tg: thyroglobulin

TGF3: transforming growth factor beta

TSH: thyroid stimulating hormone

US: ultrasound

VEGF: vascular endothelial growth factor

WHO: World Health Organization 


\section{List of papers}

This thesis is based on the following papers which, are referred to in the text by the Roman numerals I-IV.

Paper I: $\quad$ Berner A, Sigstad E, Pradhan M, Grøholt KK, Davidson B. Fine-Needle Aspiration Cytology of the Thyroid Gland. Comparative Analysis of Experience at Three Hospitals. Diagnostic Cytopathology, 2006, 34: 97-100

Paper II: Sigstad E, Heilo A, Paus E, Holgersen K, Grøholt KK, Jørgensen LH, Bogsrud TV, Berner A, Bjøro T. The Usefulness of Detecting Thyroglobulin in FineNeedle Aspirates From Patients With Neck Lesions Using a Sensitive Thyroglobulin Assay. Diagnostic Cytopathology, 2007, 35: 761-767

Paper III: Heilo A, Sigstad E, Holgersen Fagerlid K, Håskjold OI, Grøholt KK, Berner A, Bjøro T, Jørgensen LH. Efficacy of ultrasound-guided percutaneous ethanol injection treatment in patients with a limited number of metastatic cervical lymph nodes from papillary thyroid carcinoma. J Clin Endocrinol Metab, September 2011, 96: 2750-2755

Paper IV: $\quad$ Sigstad E, Paus E, Bjøro T, Berner A, Grøholt KK, Jørgensen LH, SobrinhoSimões M, Holm R, Warren D. ARG2, DDIT3, FAM129A and STT3A immunohistochemistry is not useful for the differential diagnosis of thyroid follicular tumors. Mod Pathol, 2012, 25: 537-547 


\section{Introduction}

An insignificant thyroid lesion is a frequent finding on US examination, particularly in women. Up to $72 \%$ of adult women were found to have at least 1 thyroid nodule (3). Only $4 \%$ to $5 \%$ of thyroid nodules are malignant according to histopathological examination $(4 ; 5)$. Fine-needle aspiration (FNAB) has been accepted as a first-line screening test for patients with thyroid nodules and is also widely used for examination of lymph nodes in the neck. FNAB is a safe and cost effective diagnostic procedure with accuracy in distinguishing between benign and malignant thyroid nodules approaching 94\% (6). The major limits of this procedure are the high rate of inadequate specimens (7-9) and the fact that FNAB cannot distinguish between benign and malignant follicular lesions due to identical cytomorphology (10). Clinical examination, ultrasonography and cytological examinations are all subjective methods with diagnostic limitations. Thus, it is a supposition that both the radiologist performing the ultrasonography and the cytopathologist doing on-site FNAB evaluation are dedicated and experienced. A multidisciplinary approach with experienced physicians can ensure an optimal diagnostic accuracy, as also shown in Paper I.

On-site evaluation of the FNAB specimens implies that a cytopathologist/cytotechnician participate when the FNAB is performed and immediately, at the out patient clinic, evaluate the smears. This on-site approach not only guarantees specimen adequacy, but will also aid the FNAB operator to achieve additional material for ancillary analysis. Examination of FNAB wash-out specimens may identify thyroglobulin (Tg). The present work describes how this technique may aid the clinician in diagnosing neck lymph node metastases (Paper II). Repeated neck explorations with the aim to eradicate lymph node metastases from papillary thyroid carcinoma PTC in patients with a previous thyroidectomy may be a challenge due to scar formation and also involves a greater risk of permanent recurrent laryngeal nerve 
paralysis. When doing US-guided FNAB on neck lymph nodes on these patients, an instant diagnosis of malignancy might enable the radiologist to perform percutaneous ethanol injection with the aim to cure lymph node metastases, described in Paper III.

Classical PTC is easily diagnosed on cytological smears as well as clearly metastatic cells in smears from cervical lymph nodes. In contrast to PTC, follicular carcinomas (FTC) are usually more challenging and difficult or impossible to diagnose. When a FNAB indicates follicular neoplasia, malignancy cannot be excluded. Some of these specimens may be considered to be benign, causing a delay in diagnosing FTC. The higher prevalence of large FTC with distant metastases and hence poorer survival rates than PTC patients can be attributed to the delay in diagnosing $(11 ; 12)$.

In the light of our improved understanding of molecular biology significant research efforts have been performed to identify gene expression profiles that may predict malignancy $(13 ; 14)$. However, although gene expression profiling studies have identified many possible biomarkers, the clinical application is not settled, and multicenter validation trials have to be performed to evaluate the accuracy. The disappointing results achieved when we attempted to validate four emerging biomarkers suggestive of differentiating FTA from FTC are presented in Paper IV. The role of the pathologist in management of thyroid cancer patients is changing. Figure 1 illustrates how the studies like the four included in this thesis may support the decision making. 


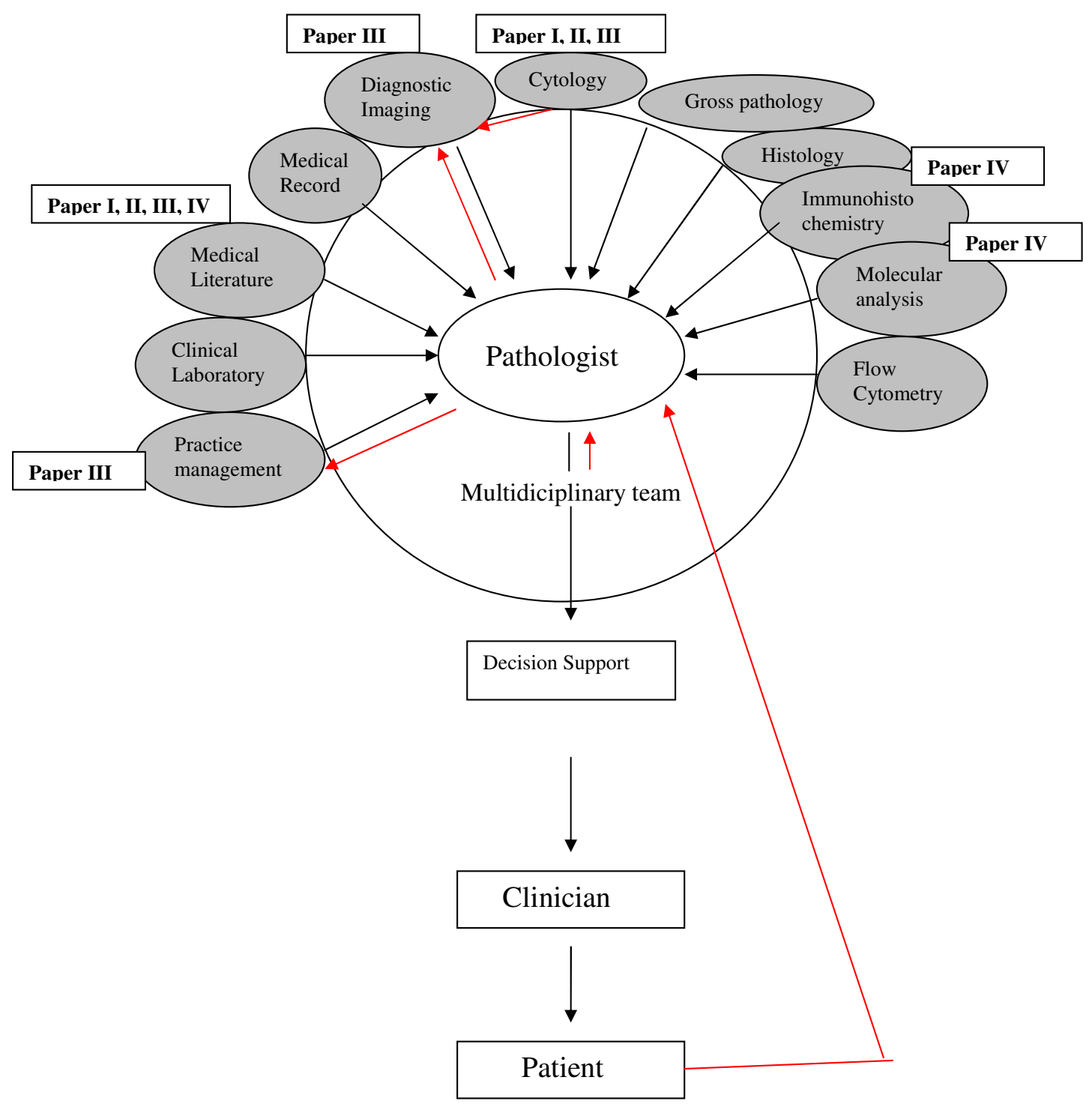

Figure 1. Modified from J. Sinard (15): The different aspects of thyroid diagnostics are visualized and the crucial position the pathologist may have making diagnoses on specimens from thyroid- and thyroid related neck lesions. The four publications in this thesis arose as a consequense of the challenges we have met making thyroid diagnoses, but also, as being member of the multidiciplinary team, I realized the challenges we have been facing in treatment of thyroid carcinoma patients. 


\section{Background}

\section{Incidence}

Clinically palpable thyroid nodules are found in $4 \%$ to $10 \%$ of the adult population in the United States and 1 of 20 clinically identified nodules is malignant $(4 ; 16)$. By ultrasound examination thyroid nodules are found in $20 \%$ to $72 \%(3 ; 17)$. By autopsy thyroid nodules are detected in approximately $50 \%$ of the glands $(18 ; 19)$. Prevalence is dependent on age, exposure to ionizing radiation, and iodine deficiency. Thyroid nodules are more frequent in women than in men.

According to WHO (20), the age standardized incidence rates per 100,000 population of thyroid carcinoma vary from 0.8 to 5.0 for males and 1.9 to 19.4 for females in different parts of the world. The highest rates have been reported from Hawaii and Iceland (WHO), whereas relatively low incidence rates have been observed in Denmark, the Netherlands and Slovakia. In the United States the lifetime risk of developing thyroid cancer is about 1:104 for men and women (21). In their study of 1985 patients Frates et al (22) detected a total of 3483 nodules larger than $10 \mathrm{~mm}$ in maximum diameter, and they found the thyroid cancer occurrence to be independent of the number of thyroid nodules. Thyroid carcinoma is the most frequent endocrine cancer but still less than $1 \%$ of human malignant neoplasms. In Europe, 200,000 patients have been treated for DTC and are survivors. The incidence of thyroid cancer in Norway is approximately two and five per 100,000 for males and females respectively (23). An estimated population of 4200 are DTC patients and survivors. The incidence of thyroid cancer has increased by $50 \%$ in the United States during the past 25 years, probably because of incidental findings of small, subclinical PTCs by neck ultrasound for nonthyroid indications, extensive used of FNAB, greater exposure to radiation after the Chernobyl 
accident and also associated with increased use of computed tomography scanning $(24 ; 25)$. A similar trend has been observed worldwide. The increased incidence of thyroid cancer in the United States is mainly assumed to be due to detection of small PTC and with no significant change in the incidence of FTC and MTC.

\section{Risk factors}

The development of the different subgroups of thyroid cancers is influenced by environmental, genetic and hormonal factors and in some cases by the interaction among them.

Among the environmental factors, ionizing radiation is the best-established cause of thyroid carcinoma. Based on five cohorts and two case-control studies Ron et al (26) found in a pooled-analysis on this issue with 58000 exposed individuals, 61000 unexposed, 700 thyroid carcinoma, and 3 million person-years, a significant dose-risk for exposure during childhood. PTCs have been shown to comprise up to $97 \%$ of the cancers occuring among atomic bomb survivors (26). After the Chernobyl nuclear power plant accident in 1986, there was reported a large increase in the incidence of childhood thyroid carcinoma in the contaminated areas (27). The risk of radiation-related thyroid carcinoma was shown to be 3-fold higher in iodinedeficient areas than elsewhere in a population-based study of thyroid carcinoma in Belarus and The Russian Federation (28).

Familial factors are extensive in the development of PTC (29). Somatic rearrangements of the RET proto-oncogene (30) and BRAF V600E mutations $(31 ; 32)$ are the most common events in PTC with BRAF mutation occurring in approximately $45 \%$ and RET/PTC is found in about $35 \%$ of adult sporadic papillary carcinomas. FTC is characterised by RAS mutations found in 
40-50\% of conventional FTC and PAX8/PPAR $\gamma$ rearrangement in approximately 35\% of conventional FTC (33). PAX/PPAR $\gamma$ tends to occur in tumors present at a younger age, in tumors of a smaller size, and also more frequently with vascular invasion. FTC occurs in up to $10 \%$ of patients with Cowden syndrome/PTEN-hamartoma tumor syndrome (34) and a few of these patients have also been found to have PTC (35).

In contrast to activation by chromosomal rearrangement in PTC, RET is activated by point mutation in MTC. Somatic mutations of the RET gene can be found in $20-80 \%$ of patients with sporadic MTC whereas germline mutations in specific functional regions of RET are found in almost all patients with familial forms of MTC (36).

In women the risk for thyroid carcinoma is approximately 3 -fold compared to men. An effect of estrogen and /or other factors associated with pregnancy have been suggested, as the incidence of thyroid cancer is almost the same for both genders before puberty and after the female menopause (37).

Rudolf Virchow was the first to describe a tumor-promoting effect of chronic irritation or inflammation (38). Inflammatory cytokines, over expressed by tumor cells, recruit haematopoietic cells such as lymphocytes, monocytes, and neutrophils into the vicinity of the tumor. Colony stimulating factors, main growth factor for i.e. monocytes, have been shown to be over expressed in different tumors (39), and these growth factors were also observed to contribute to angiogenesis. Neutrophils and mast cells both contribute to angiogenesis and remodelling of extracellular matrix $(40 ; 41)$. Leukocytes express several angiogenic growth factors including vascular endothelial growth factor (VEGF) (42), and VEGF ligands bind to their cognate receptors and induce proliferation of endothelial and/or lymphatic cells. 
Increased expression of VEGFs has been reported in thyroid carcinomas $(43 ; 44)$. Thyroid tumors have also been shown to over express epidermal growth factor receptors (EGFRs) and ligands, implicating EGFR signalling in thyroid tumorigenesis $(45 ; 46)$. The relationship between PTC and chronic lymphocytic thyroiditis (i.e. Hashimoto thyroiditis) has long been debated. The majority of recent studies have suggested that there is an increased risk of PTC in chronic lymphocytic thyroiditis (47).

Goiter and adenomas have been shown to be a major risk factor for thyroid carcinoma. According to a pooled-analysis of all case-control studies conducted before 1998, a relative risk of 30 was found in patients with a history of benign nodules/adenomas compared to 6 in women who had a history of goiter (48). The two main chromosomal patterns observed in FTC are shared with FTA, supporting the hypothesis that some adenomas may give rise to FTC through additional mutational events (49). In a study of 294 patients with multinodular goiter and thyroids surgically removed, the incidence of malignancy was almost $11 \%$ (50), which is double the incidence of thyroid cancer in the normal population.

Chronic iodine deficiency is known to be a risk factor for goiter and FTC $(37 ; 51 ; 52)$. Iodine supplementation programs, on the other hand, inducing iodine excess might, according to some epidemiological studies, increase the incidence of PTC (53-55). However, when the effects of supplementation are registered, the diagnostic improvements will follow, meaning that the increase in incidence probably is due to diagnostic improvement, and not biological factors $(54 ; 56)$. 


\section{The metastatic/infiltrating process}

Extracellular environment and thyroid carcinogenesis/tumorigenesis

Steven Paget's seed and soil hypothesis dates back to 1889, and still holds forth today (57).

He made the proposal that metastasis depends on cross-talk between selected cancer cells (the "seed") and specific organ microenvironments (the "soil").

Epithelial-stromal interaction is playing a critical role not only for normal organogenesis but also for carcinogenesis, and the interaction between cancer cells and their microenvironment can largely determine the phenotype of the tumor (58-60). In a review by Chaffer et al (61) this statement was made: "From a therapeutic standpoint, understanding the mechanisms involved in physical translocation of a cancer cell from primary tumor to the microenvironment of distant tissues is likely to be important for preventing metastasis in patients who are diagnosed with early cancer lesions, whereas understanding the mechanisms leading to successful colonization may lead to effective therapies for patients with alreadyestablished metastases". Both regulations of transmembrane receptors and of extracellular matrix have an influence on cell behaviour affecting viability, proliferation, adhesion, and motility. Tumor progression is made possible through the alteration induced by the cancer cells on the adjacent stroma to form a permissive and supportive environment (desmoplasia). Angiogenesis is also involved in formation of the desmoplasia (62).

Tumor stroma consists of a framework of extracellular matrix as well as fibroblasts, immune and inflammatory cells, fat cells and blood-vessel cells. Cancer cells may produce stromamodulating growth factors (figure 2) like fibroblast growth factors, $\beta$-catenin, endothelial growth factor (VEGF), platelet-derived growth factor, epidermal growth factor receptor (EGFR) ligands, interleukins, colony-stimulating factors and transforming growth factor- $\beta$ 
(TGFß) (58). These and other factors activate surrounding stromal cell types like fibroblasts, smooth-muscle cells and adipocytes. Activated fibroblasts promote tumor progression by secreting growth factors and pro-migratory extracellular-matrix components (58). In this epithelial-mesenchymal transition (EMT) process epithelial cells can convert into mesenchymal cells (figure 2). A series of events occur in EMT. Epithelial cells lose many of their epithelial characteristics and take properties that are typical of mesenchymal cells. Complex changes in cell architecture and behaviour are required. The molecular mechanisms regulating EMT are considerably overlapping with those that control cell adhesion, motility invasion, survival and differentiation (63). EMT is induced by interplay of extracellular signals, including components of the extracellular matrix, and also of soluble growth factors (members of the TGFß, fibroblast growth factor-FGF and epidermal growth factor-EGF). EMT has a central role in tumor progression $(64 ; 65)$. The carcinoma cells acquire mesenchymal gene-expression patterns and properties during progression to metastatic competence. With changes in adhesive properties, activation of proteolysis, and motility, which allowing the tumor cells to metastasize and establish secondary tumors at distant sites (66). Vasko and co workers (67) have shown that an expression profile consistent with EMT is common in PTC invasion.

Loss of cell-cell contact promotes tumor invasiveness. E-cadherin is a transmembranous calcium-dependent cell-cell adhesion molecule, which is highly expressed in benign thyroid tissue. The expression of E-cadherin have been shown to be low or absent in widely invasive, recurrent or metastatic thyroid carcinomas, whereas the expression in some well-differentiated thyroid carcinomas, including minimally invasive lesions is maintained $(68 ; 69)$. Liu et al (70) found that the expression of E-cadherin was decreased in the cell membrane of the tumor cells with loss of cellular polarity/cohesiveness in the invasive front of papillary thyroid carcinoma. 
In their study a group of PTCs with loss of cellular polarity/cohesiveness had extra thyroid invasion and lymph node metastases more frequently than a group without loss of cellular polarity/cohesiveness. The loss of cellular polarity/cohesiveness (LOP/C) resulted in a high recurrence risk of PTC. These results are in line with the observations made by Bai and coworkers (71) in a study demonstrating a significant correlation between the loss of cellular polarity/cohesiveness in the invasive front and the up-regulation of the extracellular matrix protein, periostin, in tumors. Both of them significantly correlated with extra thyroid invasion, pT and lymph node metastasis.

Low-density lipoprotein receptor-related protein (LRP1B) is among the 10 most significantly deleted genes across 3312 human cancer specimens. H Prazeres et al (72) recently showed that LRP1B is a key tumor suppressor through constraining the abundance of critical members of proteolytic systems in the tumor microenvironment. Inactivation of LRP1B was shown to result in changes to the tumor environment that conferred thyroid cancer cells and increased growth and invasive capacity. Restoration of inactivated LRP1B impaired tumor growth, inhibited cell invasion and led to a reduction of matrix metalloproteinase-2 (MMP-2) in the extracellular medium. 


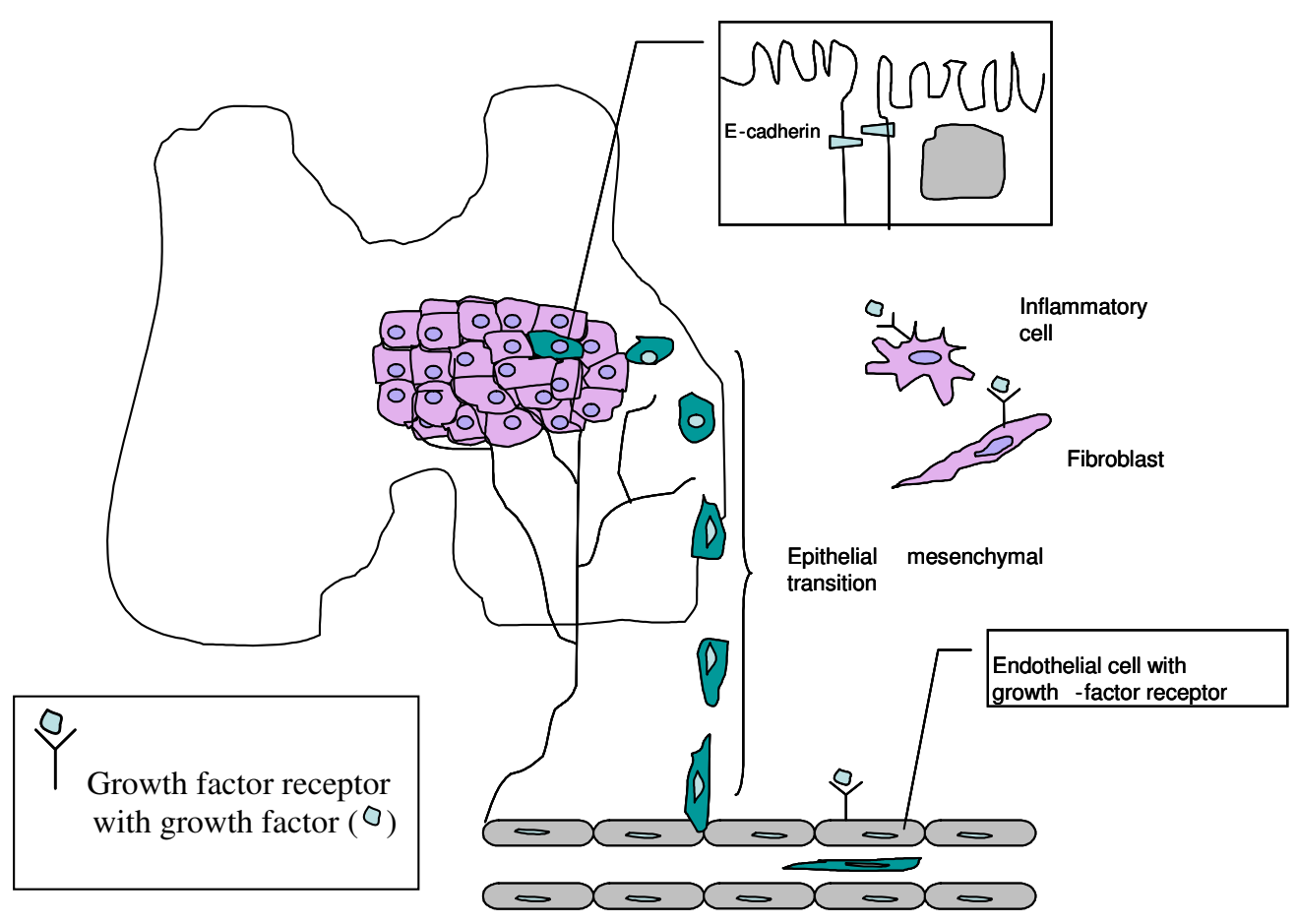

Figure 2. A malignant epithelial tumor is situated in the left lobe and isthmus of the thyroid gland. Some of the tumor cells are undergoing a transition thus acquiring properties of mesenchymal cells. These cells are able to invade vessels and become metastatic foci. The extracellular matrix is modified both by growth factors and proteases produced by the tumor and the activated fibroblasts, and inflammatory cells in the stromal surrounding secret additional growth factors and proteases. E-cadherin belongs to a family of single-transmembrane calciumdependent cell-cell adhesion proteins. E-cadherin is highly expressed in normal thyroid, benign adenomas and is also maintained in some well-differentiated thyroid carcinomas. E-cadherin is low or absent in recurrent or metastatic, and widely invasive thyroid carcinomas $(68 ; 69)$.

PTCs have a propensity for intrathyroidal spread via lymphatic vessels with further lymphagenous metastases to the regional lymph nodes, whereas FTCs metastasize mainly hematogenously. The mechanisms involved are not fully understood. Lympangiogenic growth 
factors have been shown to be significantly increased solely in PTC, whereas the expression of different factors promoting de novo blood vessel formation (hemangiogensis) is increased in all thyroid tumors (73-76).

\section{Types of thyroid malignancies and classification}

Table 1. Histopathology classification of malignancies of the thyroid gland according to WHO (20)

\begin{tabular}{|l|l|}
\hline Thyroid carcinomas & $\begin{array}{l}\text { Other malignant thyroid } \\
\text { neoplasms }\end{array}$ \\
\hline Papillary & $\begin{array}{l}\text { Primary lymphoma and } \\
\text { plasmacytoma }\end{array}$ \\
\hline Follicular & Angiosarcoma \\
\hline Poorly differentiated & Secondary tumours \\
\hline Undifferentiated & \\
\hline Squamous cell & \\
\hline Mucoepidermoid & \\
\hline $\begin{array}{l}\text { Sclerosing mucoepidermoid carcinoma with } \\
\text { eosinophilia }\end{array}$ & \\
\hline Mucinous & \\
\hline Medullary & \\
\hline Mixed medullary and follicular cell & \\
\hline Carcinoma showing thymus-like differentiation & \\
\hline & \\
\hline
\end{tabular}

The histopathology classification of malignancies of the thyroid gland according to WHO is shown in Table 1. In humans, most thyroid carcinomas arise from glandular epithelium (20) and up to $95 \%$ of tumors are well-differentiated PTCs, FTCs or MTCs, less than 5\% of carcinomas are undifferentiated (anaplastic). PTC represents approximately $80 \%$ of the welldifferentiated carcinomas, FTC 10-15\%, and approximately 5\% of tumors are MTC. MTCs originate from the thyroid $\mathrm{C}$ cell that secretes calcitonin. FTC is distinguished from adenoma only by recognition of invasion or metastasis. FTCs are divided into groups reflecting the 
biology of tumor growth and metastasis. Widely invasive FTCs, which are usually identifiable as invasive grossly, have a poor prognosis with a $25-45 \%$ 10-year survival. Patients with FTC with minimal capsular invasion have an excellent prognosis with 10-year survival rates of 70$100 \%$ (77). Survival is better in women and in patients younger than 40 years (78).

Approximately five percent of malignant tumors are lymphomas and metastases (20). Metastases to the thyroid gland most frequently arise from malignant tumors in kidney, lung and skin (malignant melanoma) (20). In Alpine regions (Switzerland) angiosarcoma has been reported to represent approximately 4\% of all thyroid malignancies during 1962-1973 (20). Endemic goiter associated with iodine-deficiency has been considered a predisposing factor (79). Angiosarcoma is extremely rare in other areas with only sporadic cases being reported (80-82).

Oncocytic change is defined as cellular enlargement characterised by an abundant eosinophilic granular cytoplasm as a result of the accumulation of altered mitochondria. The identification of oncocytic change in thyroid tumors has led to major controversies. Some lesions that were called benign developed metastases, and therefore some physicians proposed that all oncocytic tumors of the thyroid gland should be treated as malignancies (83). However, numerous studies have indicated that the criteria that apply to follicular tumors of the thyroid also distinguish malignant from benign Hürtle cell lesions (84).

Tall cell, columnar cell, diffuse sclerosing, solid/trabecular, and insular variants of PTC are all potentially more aggressive than conventional PTC varieties (85). Akslen and LiVolsi (86) found, however, that although histologic variants seemed to be significant factors on univariate survival analysis, only greatest tumor dimension and histologic grade remained as 
independent and significant predictors of carcinoma deaths. Histologic category (ie. tall cell) was found to have no independent impact on patient survival. Features recognized by other staging systems associated with unfavourable prognosis are also found present in aggressive variants of PTC, including presence of distant metastases, extracapsular spread, higher histologic grade and large tumor size. Prognosis has been found to be directly related to these factors (85).

Papillary thyroid microcarcinoma is defined as any focus measuring $\leq 1 \mathrm{~cm}$ (87). The clinical management of papillary thyroid microcarcinoma is controversial $(88 ; 89)$. Although all are clinically indolent tumors that pose little or no risk to the patient's survival and require little or no therapy, some of these patients have recurrences and other adverse outcomes that may be difficult to foresee when the diagnosis is first made.

\section{The evaluation of thyroid nodules and neck lymph nodes}

According to European consensus for the management of patients with differentiated thyroid carcinoma of the follicular epithelium (90) thyroid ultrasound (US) is the most accurate imaging detection technique. In addition to accurately describing the size and character of the nodule, US guidance is of crucial importance when performing fine needle aspiration biopsy (FNAB) (90). Furthermore, when a malignant diagnosis is made, US evaluation of neck lymph nodes is mandatory in the preoperative staging, and US-guided FNAB of sonographically suspicious lymph nodes should be performed to confirm malignancy (91).

The Bethesda System for Reporting Thyroid Cytopathology (92) is widely in use. The morphological criteria for clearly benign and malignant thyroid lesions are easy to interpret and the report from the cytopathologist is easy to comprehend for the clinicians. In contrast, 
smears from follicular lesions are difficult to diagnose, due to the fact that only capsular and/or vascular invasion, detected only by histologic evaluation, can distinguish between FTA and FTC, and additionally the diagnosis of follicular variant of PTC may also be challenging to distinguish due to the subtlety of diagnostic nuclear features. In the Bethesda System these lesions are reported as "Follicular lesions of undetermined significance". On-site adequacy assessment reduces the number of inadequate samples (93-96). The on-site evaluation also allows the cytopathologist to achieve important clinical information and is a prerequisite for the multimodal approach.

Patients with PTC present with cervical lymph node metastases at the time of primary diagnosis in $20-90 \%$ of the cases, and more frequently than other histotypes (97). In 10-26\% of all patients with well-differentiated thyroid carcinoma, cervical lymph node metastases present as the first sign of disease (98). According to the "European consensus for the management of patients with differentiated thyroid carcinoma of the follicular epithelium" (90) a thorough examination of the neck lymph nodes should be performed as part of a preoperative staging, and FNAB should be performed in cases where the lymph node presents as suspicious on ultrasound. In the follow-up of patients after treatment for thyroid carcinoma all suspicious neck lesions should be punctured for cytological examination. The cytological specimen from lymph node metastasis is in some cases sparse and suboptimal, as for many cystic lesions, and $\mathrm{Tg}$ measurement of wash-out FNAB needle specimen, might increase the sensitivity (99). 


\section{Diagnostic markers for discriminating benign and malignant tumors}

\section{Immunochemical markers}

Diagnostic immunohistochemical thyroid markers were introduced through the study of keratin and thyroglobulin in the early 1980ties (100). Since then, studies have been performed with the aim to identify new markers to distinguish benign from malignant thyroid tumors. Among these markers are CK 19, GAL3 and HBME1. Potentially new markers have also been tested on cytological specimens. Some studies have included specimens categorized as suspicious for follicular neoplasia, in which overlapping cytological features makes it difficult to separate a follicular carcinoma from an adenoma. De Matos and co-workers (101), used the three markers CK 19, GAL3 and HBME1 together and demonstrated in a clinical study population that it was possible to make the diagnosis of PTCs in 81/84 (96.5\%) and of FTCs in 24/38 (63.1\%). A review by Sanabria et al (102) concluded that "galactin-3 is not an absolute marker for identifying malignancies on cytologically indeterminate thyroid nodules (follicular pattern), although it could be used as a useful supplementary marker". A study by de Micco et al (103) confirmed that immunocytochemistry with thyroid peroxidase is better than HBME1 for diagnosis of malignancies on FNAB from the thyroid gland. Griffith et al (104) have in an expert review evaluating studies presenting IHC biomarker panels concluded that "the use of diagnostic biomarker panels has shown only marginal improvement in performance compared with the best individual biomarkers themselves". The information achieved from IHC testing will, when promising markers are found, be applied to FNAB specimens. There are, however, still many pitfalls when performing immunocytochemistry, and no "magic" markers has been found. 


\section{$\underline{\text { Molecular markers }}$}

The mitogen-activated protein kinase (MAPK) pathway (figure 3) in cancer progression and in promotion of cancer growth is well established (105). Mutations in this pathway are found in $>70 \%$ of papillary carcinomas involving RET/PTC rearrangement and point mutations of the BRAF and RAS genes. Mutations of the BRAF gene have been found in a variety of human cancers. In thyroid tumors, the BRAF mutation occurs only in papillary carcinomas and a subset of anaplastic thyroid carcinomas. The prevalence of BRAF V600E mutation has ranged from $29 \%$ to $83 \%$ in different series of PTCs $(106 ; 107)$. BRAF mutations have not been detected in any FTC (108). Several studies have demonstrated a strong association between BRAF mutation and poor clinicopathological outcome $(109 ; 110)(111)$. A close relationship has been demonstrated between BRAF mutation and extra thyroidal extension, lymph node metastasis and advanced TNM stage of PTC, and these factors have been associated with increased rates of recurrence and mortality of thyroid cancer (112-114). BRAF mutation is most commonly associated with the aggressive tall-cell variant and the classic variant of PTC and least commonly with the less aggressive follicular variant of PTC (115). However, Cheng and co-workers (116) found that within each morphologic subtype, BRAF mutation alone did not predict aggressive behaviour. Their data emphasized the importance of detailed histotyping in parallel to proteomic and mutational characterization in defining cancer behaviour. The evaluation of BRAF mutation status preoperatively may assist in the decision-making in diagnosis, on initial surgical management, on initial radioiodine treatment and in follow-up of patients with PTC. 


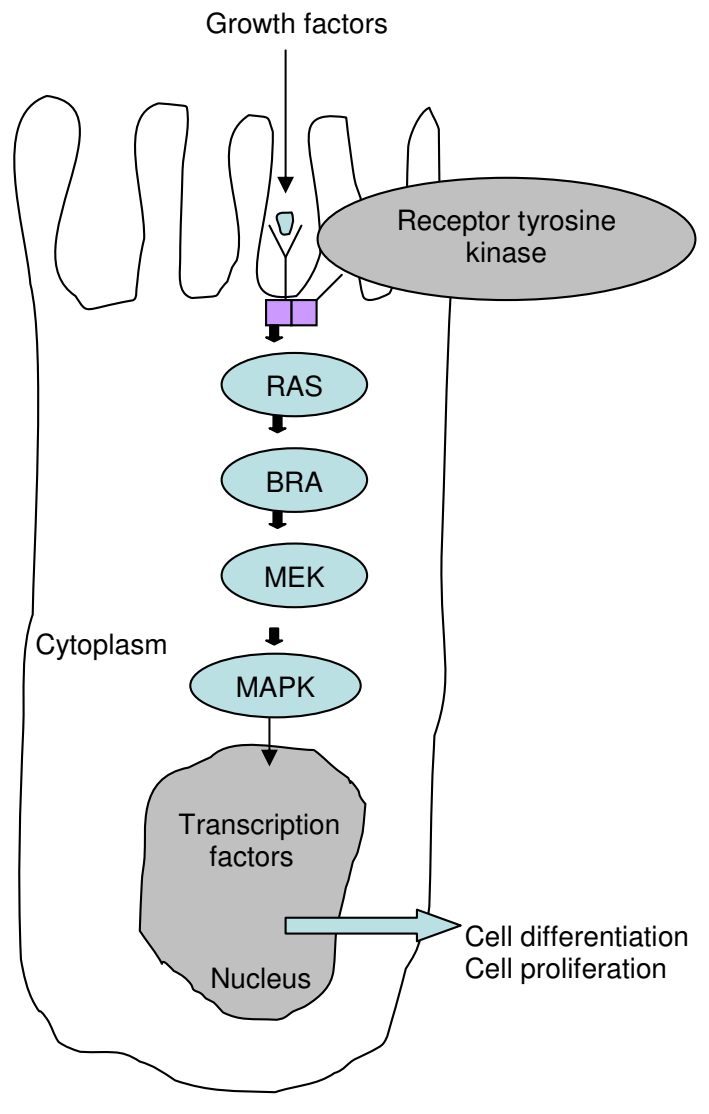

Figure 3:

Key components of the MAPK/ERK pathway. Epidermal growth factor binds to EGF receptor in the cell membrane, starting the cascade of signals. Signals enter the cell nucleus and causes transcription of DNA which is then expressed as protein. The mutation of the BRAF gene results in constitutively activation of the pathway causing tumorigenesis.

Unlike PTC, RET and BRAF do not play any role in the pathogenesis of FTCs. FTCs show a predominance of RAS mutations, PAX8-PPAR $\gamma$ rearrangements and aneuploidy. PAX8PPAR $\gamma$ rearrangement is found in 30-40\% of FTCs of conventional type (117). Additionally PAX8-PPAR $\gamma$ rearrangement has been detected in a high percentage of cases $(37.5 \%)$ in follicular variant of PTC (FVPTC) $(118 ; 119)$ whilst the prevalence in oncocytic carcinomas is 
lower $(120 ; 121)$. PAX8-PPAR $\gamma$ rearrangement induces a strong over expression of PPAR $\gamma$ protein (122). However, the cellular impact of PAX8-PPAR $\gamma$ rearrangement in thyroid is not yet fully characterized. A recent meta-analysis of all FTC outcomes data reported in the literature, indicated that $68 \%$ of FTC-expressing PAX8-PPAR $\gamma$ fusion protein were minimally invasive, suggesting that most PAX8-PPAR $\gamma$ fusion protein-expressing tumors were more likely to be minimally invasive than tumors that fail to express this fusion protein (123). The PPAR $\gamma$ protein can be detected by immunohistochemistry $(122 ; 124)$.

Point mutations of the three RAS genes, H-RAS, K-RAS and N-RAS are not restricted to a particular type of thyroid tumor and are found in FTCs (40-50\%), PTCs (10-20\%), and FTAs (20-40\%) (33). PTCs harbouring RAS mutation almost always have the follicular variant histology $(125 ; 126)$. The three genes encode highly related $21-\mathrm{kDa}$ proteins located at the inner surface of the cell membrane participating in the transduction of signals arising from tyrosine kinase and $\mathrm{G}$ protein-coupled receptors. When bound to guanosine triphosphate (GTP), MAPK and other signalling pathways, such as PI3K/AKT, are activated. As a result of the point mutations in the specific domains of the RAS genes, the mutant protein becomes permanently switched in the active position, thus chronically stimulation downstream targets (121). RAS mutations are not suitable as diagnostic marker for FTC due to occurrence with significant prevalence in FTA and the follicular variant of PTC.

\section{Treatment of differentiated thyroid carcinoma and metastases}

\section{Surgery}

The standard surgical treatment of well-differentiated carcinoma is total (or near-total) thyroidectomi (90). For patients with well differentiated solitary carcinomas less than $1 \mathrm{~cm}$ in diameter with no evidence of nodal or distant metastases, and no history of previous radiation 
exposure, lobectomi is considered sufficient (90). Metastases to loco-regional lymph nodes has been found in $20-90 \%$ of patients with PTC (97). Consequently prophylactic bilateral central neck dissection to remove potential lymph node metastases has been recommended on patients with a preoperative diagnosis of PTC $(127 ; 128)$. Patients with lymph node metastases in the lateral neck compartment should be treated with en-bloc resection of the involved compartment rather than selective dissection ("berry-picking") to reduce the risk of local recurrence (129). In patients who have undergone lobectomy or subtotal thyroidectomy, a completion thyroidectomy is advised in the cases with a potential for recurrence. These cases include PTCs with a diameter larger than $1 \mathrm{~cm}$, multifocal tumours and FTCs demonstrating more than minimal capsular invasion (130).

Therapeutic dissection of positive lymph nodes identified on physical examination and confirmed by ultrasonography or FNA cytology/Tg-FNAB of a patient with PTC, decrease the incidence of loco regional recurrence, prevent serious sequelae and possibly improve survival $(131 ; 132)$. The role of elective prophylactic central compartment lymph node dissection for overt nodal metastases remains a matter of debate $(132 ; 133)$. Some studies have shown that performing a central lymph node dissection in addition to total thyroidectomy has resulted in an increased rate of transient hypoparathyroidism (134-136). Revision surgery of the central compartment increase the risk of permanent injury to the parathyroid glands, and have been reported ranging from $0 \%$ to $9 \%(137)(138 ; 139)$.

\section{$\underline{\text { Percutaneous ethanol injection (PEI) treatment }}$}

PEI treatment has been introduced as an alternative to surgical resection of lymph node metastases from PTC (140). However, the numbers of PEI treated lymph nodes are small and the results have to be confirmed in larger series. 


\section{$\underline{\text { Radioiodine (RAI) Ablation }}$}

Thyroid epithelial cells are capable of intracellular concentrating iodide as a substrate for thyroid hormone synthesis, which is a prerequisite for the unique radioiodine treatment. In Western countries radioactive iodine in the form of ${ }^{131} \mathrm{I}$ has since 1946 been administrated postsurgical to destroy possible thyroid residue in the thyroid bed (thyroid ablation). There are several reasons for such thyroid remnant ablation. RAI may destroy residual microscopic metastases and normal thyroid tissue destined to become malignant (141). A few years ago, RAI ablation was recommended routinely after total thyroidectomy in order to get better control with the Tg measurements. Additionally, a large remnant may obscure ${ }^{131}$ I uptake in cervical or lung metastases. However, RAI ablation is clearly associated with acute and longterm side-effects. In a recent time trend analysis of RAI used in a cohort of 189219 patients with DTC treated in US between 1990 and 2008, a significant increase in the proportion of patients with DTC receiving RAI-treatment was found (142). According to this study, more than a third of patients with low-risk tumors received RAI. The most common chronic side effect after RAI treatment is reduced saliva production (143). A major risk of RAI therapy is bone marrow suppression (144). The risk of secondary primary malignancy has been shown to be increased after RAI exposure, and a strong relationship has been found between cumulative dose of RAI and risk of cancers of bone and soft tissue $(145 ; 146)$. There is also an increased risk of developing leukaemia $(141 ; 146)$. The benefits of using RAI in low-risk patients are debated $(147 ; 148)$, and according to the newly established guideline by Japanese Society of Thyroid Surgeons and Japanese Association of Endocrine Surgeons, RAI is not recommended to low-risk patients due to excellent prognosis (149). This is in line with the revised American Thyroid Association Management Guidelines (91) not recommending RAI ablation for patients with unifocal cancer $<1 \mathrm{~cm}$ without other higher risk features. 


\section{Thyroid Hormone Suppression of TSH}

According to European consensus for the management of patients with DTC (90) is TSHsuppressive therapy mandatory in patients with evidence of persistent disease (including detectable serum $\mathrm{Tg}$ and no other evidence of disease). The risk of recurrence in low-risk patients subsequent to a cure has been reported to be low $(<1 \%)$. The aim for serum TSH level is within the lower part of the normal range (between 0.5 and $1.0 \mathrm{mU} / \mathrm{l})(150)$.

\section{External Radiation Therapy}

External radiotherapy is administered to patients with undifferentiated (anaplastic) thyroid carcinoma. Some studies have demonstrated that external radiation therapy may improve loco regional control in patients with nonanaplastic thyroid cancer who have high risk features $(151 ; 152)$.

\section{Potential approaches for new therapies of thyroid cancer}

Thyroid-specific gene expression, including the ability to take up and organify radioiodine and to make thyroglobulin is lost during the dedifferentiation process. However, new therapeutic approaches to thyroid cancer are developed due to advances in molecular and cellular biology. Among therapeutic approaches established in vitro for restoration of differentiated thyroid function are redifferentiating agents and gene therapy. A multikinase inhibitor SU12248 (Sunitinib) has been shown to effectively inhibit signalling from RET/PTC kinase in experimental models and has been progressed to phase II clinical trial in radioiodine-refractory, unresectable DTC and MTC (153). BAY 43-9006 is another multikinase inhibitor which inhibits the BRAF signalling and growth of all thyroid cell lines carrying the mutant BRAF (154). A longer than 16-week phase II trial of BAY 43-9006 
(sorafenib) in 30 patients with metastatic iodine-refractory thyroid carcinoma showed an overall clinical benefit of $77 \%, 70 \%$ with thyroglobulin reduction, and a median 79 -week progression-free survival (155). Another phase II trial of sorafenib in patients with metastatic thyroid cancer also showed a similar antitumor activity, with a median progression-free survival of 15 months(156). There is, however, to date no evidence to show the antitumor effects of sorafenib are through the inhibition of BRAF. Sorafenib may target other kinase pathways such as VEGFR to inhibit tumorigenesis. Unfortunately, sorafenib has a range of side effects that must be taken into consideration prior to the initiation of therapy. The most common adverse events reported include diarrhea, hypertension, fatigue and hand-foot syndrome $(155 ; 156)$. Vandetanib, a small molecule tyrosine kinase inhibitor, targets VEGF receptors, RET, and at higher concentrations, the EGF receptor $(157 ; 158)$. Vandetanib has previously shown antitumor activity in a phase II study of patients with advanced hereditary MTC (159).

\section{Prognosis}

PTC represents about $80 \%$ of all thyroid cancers. It typically follows an indolent course with a 10 years cause-specific survival rate of young patients ( $<45$ years) over $98 \%$ and $90 \%$ regardless of age (20). For minimally invasive FTC, long term mortality is in the range 3 to 5\%. Matched for sex and age the survival curves approach those of a normal population (20). A number of clinicopathologic prognostic factors such as older age, larger tumor size, extra thyroidal invasion, lymph node and distant metastases, and aggressive tumor histologic subtypes such as tall cell, columnar, diffuse sclerosing variants and insular variant are associated with worse disease-free overall survival (78). 
Molecular testing is well-suited as an adjunct to morphological examination because it requires a relatively small amount of tissue. The use of molecular markers, in conjunction with conventional clinicopathological risk factors might assist the prognostication of thyroid carcinoma and improve the efficiency of contemporary management of thyroid cancer. The possibility of predicting the biological behaviour of PTC is growing. Testing a FNAB specimen demonstrating PTC for a molecular marker profile that could accurately stratify risk and prognosis would be ideal. BRAF V600E mutation in PTC predicts a poorer prognosis (160) and a test for BRAF preoperatively can help tailoring initial and reoperative surgery. A recent study has showed that molecular testing for a panel of mutations may be particularly useful in nodules with indeterminate cytology, especially in the lowest risk subgroup of indeterminate cytology (161).

\section{Diagnostic follow-up after initial treatment}

Neck US-examination and serum thyroglobulin measurements are the primary and standard follow-up procedures for differentiated thyroid cancer $(90 ; 91)$. This procedure may detect lymph nodes as small as $2 \mathrm{~mm}$, but the procedure is operator-dependent. US-guided FNAB with immediate on-site cytological evaluation might confirm the suspicion of metastasis. In cases where the cytological specimen is inconclusive, $\mathrm{Tg}$ measurement of the wash-out specimen will be able to confirm metastasis in cases where morphology is not conclusive for malignancy $(99 ; 162 ; 163)$.

The measurement of serum $\mathrm{Tg}$ is crucial in the diagnostic follow-up. The method is specific and extremely useful. The presence of serum anti-Tg antibodies ( $\mathrm{Tg}-\mathrm{Ab}$ ) must be determined concomitantly, as circulating anti-Tg antibodies may interfere and cause a false negative serum $\mathrm{Tg}$ determination $(164 ; 165)$. 


\section{Aims of the thesis}

\section{General aim}

The main objective of this study was to analyze the diagnostic accuracy of FNAB in thyroid nodules. Furthermore to establish new reliable procedures for follow-up of patients with well differentiated thyroid carcinoma.

\section{Specific aims}

1. To evaluate the cytopathologists contribution in a multidisciplinary thyroid team.

2. To examine if $\mathrm{Tg}$ measurements in needle wash-out specimens from suspicious neck lymph nodes increase the detection rate of metastases from PTC.

3. To validate the effect of PEI on neck lymph node metastases from PTC including evaluation of the combined use of cytology and Tg measurements in needle wash-out specimens.

4. To examine the accuracy of four selected immunomarkers, DDIT3, STT3A, ARG2 and FAM129A to differentiate between FTC and FTA. 


\section{Material and methods}

Table 2. Study material

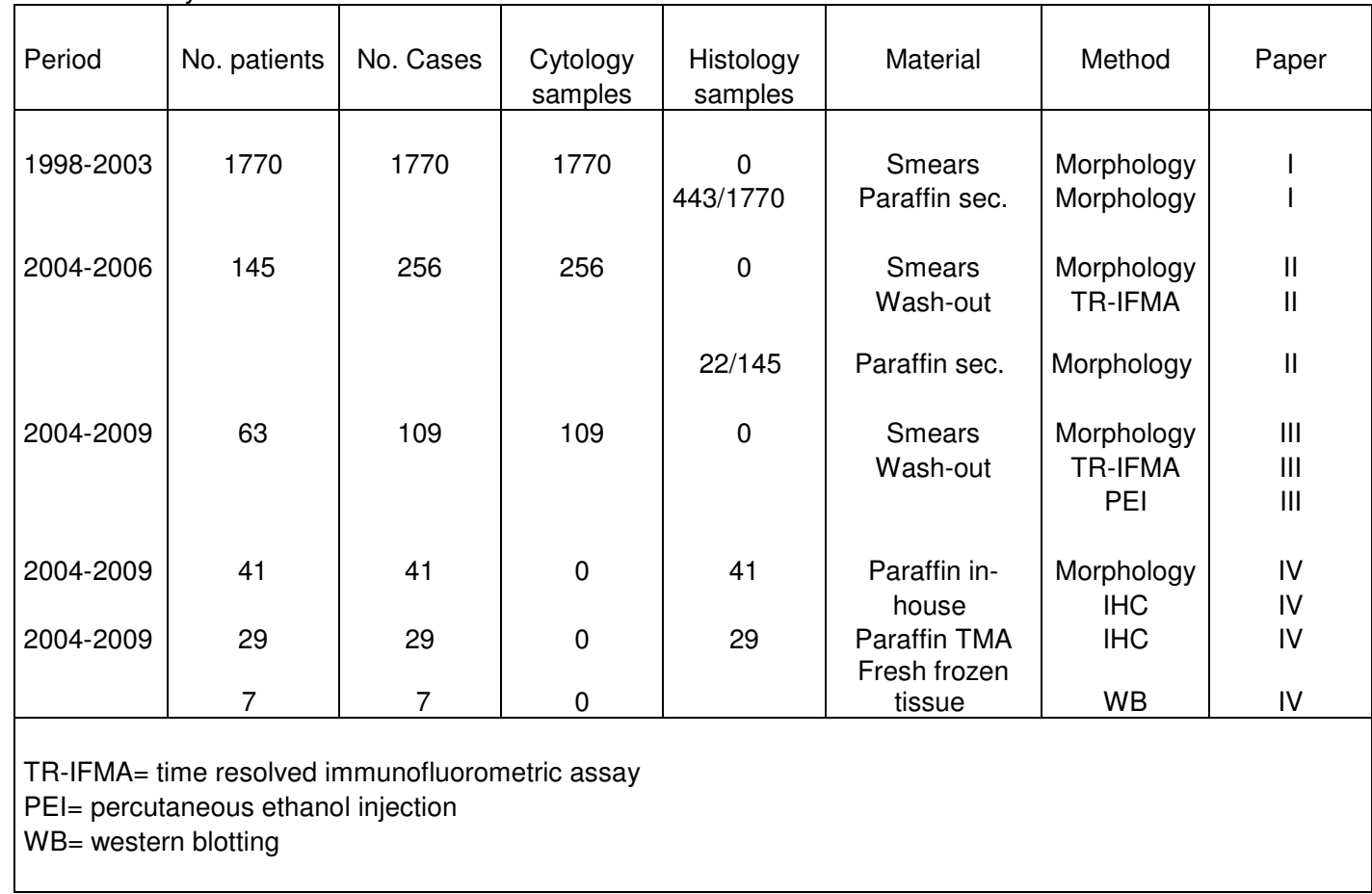

\section{Paper I}

\section{$\underline{\text { Specimens }}$}

The reports of 1770 FNAB specimens diagnosed at the Norwegian Radium Hospital (NRH) between 1998 and 2003 were obtained for the comparative analysis of experience at three hospitals. Four hundred and forty-three of the 1770 cases were excised for histological examination (Table 2). Of the 1770 specimens, 254 were obtained at the Norwegian Radium Hospital and 1516 were submitted from other hospitals including 424 from two local hospitals 
evaluated in the present study (320 specimens from Hospital A and 104 specimens from Hospital B).

\section{Morphology}

Criteria for reporting cytology and histology diagnoses were benign, suspicious, malignant, or unsatisfactory.

\section{$\underline{\text { Technical Considerations }}$}

At all three hospitals two steps of the FNAB procedure were the same: 1) clinical examination and localization of the target lesion and 2) ultrasound-guided sampling using a 25-gauge or 27-gauge needle without suction. At NRH an experienced cytopathologist participated in the FNA procedure and performed the third step: preparation of air-dried, fixed, and Giemsastained smears for immediate on-site microscopy, and the possible need for additional techniques. At community Hospitals A and B radiologists/technical staff performed all three steps, with the exception of evaluation of adequacy. At hospital A a cytopathologist was evaluating slides from some of the cases on-site, but the pathologist did not participate actively during performance of the puncture as was the case at NRH. Reports were obtained from the electronic pathology database (Doculive). Four hundred and forty-three of the 1770 lesions were subsequently excised and the histological data from these cases were found registered in the same database. All histological specimens were fixed in formalin and embedded in paraffin blocks from which HE-stained sections were made. 


\section{Paper II}

\section{$\underline{\text { Specimens }}$}

A total of $256 \mathrm{Tg}$-FNAB samples from 145 patients were investigated for the assessment of the diagnostic utility of thyroglobulin ( $\mathrm{Tg}$ ) in fine needle aspirates (Tg-FNAB) of nonthyroidal neck masses. All patients had one or more suspicious or indeterminate cervical lymph nodes in neck ultrasound. The Tg-FNAB values were compared to corresponding S$\mathrm{Tg}, \mathrm{S}-\mathrm{TgAb}$, morphology of cytological smear and to histology of 46 surgical specimens.

\section{Morphology}

Cytological smears from lymph nodes were classified as inadequate or not diagnostic, cells suspicious for DTC metastases, cystic material with macrophages and/or irregular epithelial cells suspicious of metastasis from papillary carcinoma or finally as malignant (metastasis from papillary thyroid carcinoma or other malignancies). Histologic diagnosis was performed according to WHO classification (20).

\section{$\underline{\text { Technical Considerations }}$}

All FNAB specimens were obtained with a 27- or a 25-gauge needle under ultrasound guidance and a free hand puncture technique without suction. Cytological smears were made, air-dried and stained with DiffQuick. The sample needle was immediately rinsed with $500 \mu 1$ matrix buffer or $0,9 \%$ saline. Washouts were subsequently subjected to thyroglobulin measurement. Time resolved immunofluorometric assay (TR-IFMA) for Tg was performed in the Department of Medical Biochemistry, Rikshospitalet-Radiumhospitalet (today included in Oslo University Hospital), and was based on two monoclonal antibodies and performed in 
streptavidin-coated 96 well microtiter plates. Monoclonal antibodies were raised essentially as described for neuron specific-enolase (NSE) (166) using purified Tg from a human thyroid gland as antigen. Tg antibodies were determined by Anti-TGn or Anti-TGn Kryptor from BRAHMS (Berlin, Germany).

\section{Immunoassay}

Immunoassay is an analysis used to protein detection utilizing the interaction between a known antibody and the antigen (analyte) of interest. The antibody has to be labelled in a way that is easily detectable. The antigen should be detected both when it is present in low concentrations and also when it is present in excess. The background may be a challenge, especially when the antigen is present in low copy numbers. This is the case in biological samples containing a huge number of different proteins. Time-resolved immunofluorometric assay offers an ideal way of removing unwanted background fluorescence with a consequent increase in sensitivity. A fast light pulse is used to exite a fluorescence probe whose exited state has a decay time of between 100ns and $1 \mu \mathrm{s}$. The non-specific background fluorescence with a decay time of less than 10ns is discriminated against by using a gated detection system witch measures the fluorescence after the background decay is reduced almost to zero. Luminescent lanthanide complexes have exceedingly long-lived luminescence delivering greater assay sensitivity and a broader dynamic range. The immunoreaction may occur on a solid phase (heterogeneous) or in solution (homogeneous) and the antibody-antigen binding may be monitored via displacement of a labeled antigen (competitive) or directly (noncompetitive). Sample matrix is an important source of background measurements in biological assays (serum, tissue sample), and this is particularly the case for homogeneous assays (167). Removal of interfering substances may rise the sensitivity in luminescence measurements. 
The measurement of Tg-FNAB has to be ultrasensitive to be able to detect the presence of even small amounts of Tg. The wash-out specimens from neck lymph node FNABs is often minimal, and the possibility of detecting even tiny amounts of $\mathrm{Tg}$ is crucial when exploring neck lymph nodes in patients operated for PTC. The Tg-FNAB measurements must always be compared with serum $\mathrm{Tg}$, in order to avoid false positive result due to blood contamination.

A sensitive in-house time-resolved immunofluorometric assay was performed when measuring Tg-FNAB in study 2 and 3 (described in Paper II). We used a microtitre platebased, dissociation-enhanced lanthanide fluorescence immunoassay (DELFIA) in a heterogeneous non-competitive format. In this system the detection antibodies are carrying the lanthanide, and the fluorescence are measured after a reaction including an enhancement solution. The chelated lanthanide $\mathrm{Eu}^{3+}$ was chosen to produce luminescence. To obtain minimal interference from human anti-Tg antibodies in serum when used in the assay for $\mathrm{Tg}$, hybridomas were selected in presence of autoantibodies. Additionally, the immunoreaction was carried out on a solid phase (microtitre plate) to further reduce the interference. Combined with the morphological evaluation of FNAB smears this approach to the measurements increased the assay sensitivity of the Tg-FNAB examinations and delivered a broader dynamic range.

\section{Paper III}

\section{$\underline{\text { Specimens }}$}

Samples from 124 metastatic neck lymph nodes from 69 patients who previously had undergone thyroidectomy for PTC were confirmed by US-guided cytological biopsy and or by 
raised levels of thyroglobulin (Tg) in wash-outs from cytological specimens from June 2004 through December 2009. Three patients were excluded due to lack of follow-up and three patients (eight metastases) were reassigned for surgery, leaving 63 patients with 109 lymph nodes in total for the treatment with PEI as monotherapy.

\section{Morphology}

The Bethesda classification system was used when reporting FNAB specimens (92).

\section{$\underline{\text { Technical Considerations }}$}

Measurement of Tg-FNAB were obtained and handled as described for Paper II. Cystic lesions suspicious of metastasis were in most cases punctured several times, and multiple slides were made.

PEI-treatment was performed in all the metastatic neck lymph nodes by injection of 0.1-1.0 $\mathrm{ml}$ of $99.5 \%$ ethanol guided by US. PEI was performed by one of three radiologists dedicated to US examination of the neck. The amount of ethanol injected was depending on the size of the lymph node, and in larger nodes the needle was repositioned into different parts of the node.

\section{Paper IV}

The specimens as well as the performance of immunohistochemical staining, antibody

preparation, ${ }^{125}$ I-peptide displacement assays, western blotting, production and characterization of antibodies are all described thoroughly in the paper. 


\section{$\underline{\text { Specimens }}$}

For the immunohistochemical study 30 cases from primary tumors diagnosed in the Department of Pathology at The Oslo University Hospital were included. All specimens were originally submitted for diagnostic purposes. The 30 cases consisted of 15 follicular carcinomas and 15 follicular adenomas. Follicular neoplasias with oncocytic differentiation were not included, and cells demonstrating oncocytic differentiation were not included in the evaluation. To investigate whether the staining of the normal thyroid tissue adjacent to the tumours was influenced by the staining of the tumours ("overspill"), slides from 11 cases of normal thyroid tissue from thyroid lobes not harbouring pathological findings were immunostained. Additionally one sample from a lymphoid nodule was included in this immunostaining. Tissue micro arrays (TMA) (US Biomax Inc, Rockville, USA) (www.biomax.us) were included when staining for each of the four antibodies. One set of the TMA-slides (serial TH802) contained samples from normal thyroid parenchyma and the other set (serial TH804) of slides were having samples from thyroid cases with pathological findings including eight FTCs and 21 FTAs.

\section{Morphology}

Histologic diagnosis of the in-house cases was performed according to WHO classification (20).

\section{$\underline{\text { Technical Considerations }}$}

The in-house cases and the histological specimens referred to our hospital were fixed in neutral phosphate buffered formalin and embedded in paraffin blocks from which HE-stained sections were made. The tissue specimens from Biomax were preserved in neutral phosphate buffered formalin for 24 hours. The tissue specimens in-house were preserved in $4 \%$ buffered 
formalin. The preservation time for the in-house cases was variable, but within the range of 25 days.

Antibody preparation and ${ }^{125}$ I-peptide displacement assays evaluating the affinity for the two homemade monoclonal and polyclonal antibodies as well as the Western blotting on tissue samples and cells from a follicular carcinoma line (FTC-133, EACC, Salisbury, UK) were performed at the Department of Medical Biochemistry at NRH at Oslo University Hospital. Immunohistochemical staining was performed at the Pathology Department using buffer AR10 (Biogenex, San Ramon, CA) in a steamer for 10 minutes to achieve antigen retrieval. Immunodetection was undertaken using Dako automated staining system (S3400 Dako, Carpinteria, CA, USA) using EnVision+ HRP reagents (K 4007 and K4011, Dako). The inclusion of specimens handled in two different laboratories was important to avoid preanalytical variability influencing the result. Both in-house cases and TMA-specimens were handled according to standardized preanalytical methods for formalin fixation and paraffin embedding. The laboratory staffs performing the IHC were highly experienced and the staining and immunodetection system used was automated and identical to what was used by the research group $(168 ; 169)$ presenting the results we were trying to validate (DAKO). Some proteins are relatively stable in paraffin-embedded tissue blocks but can be rapidly deteriorate once sectioned, thus duration of fixation can be of importance for the results (170). In our study, all slides were sectioned immediately before doing IHC, thus minimizing the possibility of protein deterioration. In our study endogenous peroxidase activity was suppressed by pretreatment with $0.03 \%$ hydrogen peroxide, and the background were "clean" evaluating IHC both in-house cases and TMA-specimens. 


\section{Statistical methods}

The data were analyzed using the Statistical Package for the Social Sciences (SPSS), version 170 17.0.1 for Windows (SPSS Inc., Chicago, IL, USA). 


\section{Main Results}

Paper I. Fine-Needle Aspiration Cytology of the Thyroid Gland. Comparative Analysis of Experience at Three Hospitals

The fraction of unsatisfactory FNA specimens from three hospitals with differences in skill of the performing physicians was compared. A substantially higher fraction was detected at community Hospital B where several radiologists, generally with limited experience and skill, were performing the FNAB, compared to NRH and community Hospital A $(21.2 \%, 7.9 \%$ and $5.9 \%$, respectively) employing a few dedicated radiologists.

\section{Paper II. The Usefulness of Detecting Thyroglobulin in Fine-Needle Aspirates From} Patients With Neck Lesions Using a Sensitive Thyroglobulin Assay

We found the combination of FNAB cytological examination and measurement of $\mathrm{Tg}$ in FNAB washout from cervical lymph nodes in patients with PTC to be highly sensitive and to give important information when the cytological morphology was not conclusive concerning the demonstration of metastatic cells. This was particularly the case for cystic metastases (TgFNAB was positive in 25 specimens with suspicious or cystic cytology).

Serum thyroglobulin antibodies appeared to have ignorable effect on the performance of $\mathrm{Tg}$ FNAB. The combined use of cytomorphology and $\mathrm{Tg}$ measurements in needle wash-out specimens was important in follow-up of the patients treated with PEI (Paper III).

\section{Paper III. Efficacy of ultrasound-guided percutaneous ethanol injection treatment in} patients with a limited number of metastatic cervical lymph nodes from papillary thyroid carcinoma

Metastatic neck lymph nodes in papillary thyroid carcinoma can be completely eradicated by ultrasound-guided percutaneous ethanol injections (PEI). US-guided PEI-treatment is an 
excellent alternative to surgery in patients with limited cervical nodal metastases. The PEI technique applied is an outpatient procedure and in our study only one patient needed injection of local anaesthetic during a session. All patients were discharged immediately after. The efficacy of the PEI-treatment was evaluated by US of the lymph nodes treated. Excellent indicators were disappearance of the lymph node, size reduction and reduction or absence of vascularity in the lymph nodes. The efficacy was also confirmed by the combined use of cytomorphology and $\mathrm{Tg}$ measurements in needle wash-out specimens.

\section{Paper IV. ARG2, DDIT3, FAM129A and STT3A immunohistochemistry is not useful for the differential diagnosis of thyroid follicular tumors}

This study did not succeed in an attempt to confirm the results from earlier studies $(168 ; 169)$ concluding that four biomarkers (DDIT3, STT3A (ITM1), ARG2 and FAM129A (C1orf24) were able to distinguish between follicular adenoma and follicular carcinoma. No significant difference was found when evaluating the binding of the antibodies in benign follicular adenomas compared to follicular carcinomas. Additionally, binding of these four biomarkers was also observed in normal thyroid parenchyma adjacent to the tumors and in slides from separate specimens of normal thyroid parenchyma.

The expression levels of ARG2, FAM129A, DDIT3 and STT3A in FTA and FTC biopsies using western blotting were, to a great extent, in agreement with our observations using immunohistochemistry. 


\section{Discussion}

In all four papers included in this thesis, the pathologist played an important role in collecting and evaluating the specimens.

\section{Paper I}

When we performed this study only one relevant report regarding the importance of adequate training of the person obtaining FNAB specimen was published (171). During the subsequent years great effort has focused on the field of standardizing and quality assuring of FNAB from thyroid lesions, both institutionally, nationally and internationally. National Guidelines for Management of Well-Differentiated Thyroid Carcinomas were published in 2006 (172). These guidelines are based on institutional (NRH) and national experience as well as on American and European guidelines (90;127).

This paper highlights the importance of the skills of the participating members of the multimodal thyroid group. Experience and skill of the radiologist performing the ultrasound examination of the thyroid gland and neck is obligatory to achieve representative high quality FNAB specimens. The same cytopathologists participated in the diagnostics of specimens from thyroid lesions from all three hospitals. We were able to compare the fraction of satisfactory specimens from all locations. US examination and FNAB of the thyroid and neck was, with few exceptions, performed by one dedicated and skilled radiologist at community Hospital A. At NRH two or three radiologists participated in the US examinations and FNABs, whereas at community Hospital B several radiologists were involved. The number of FNABs performed during the six year of this study was three times higher at community Hospital A ( $n=320)$ compared to community Hospital B $(n=104)$. According to these numbers, less than 20 FNABs were performed at community Hospital B annually. 
At NRH, an experienced cytopathologist always participated in the FNAB procedure and immediately took care of the specimen for correct smearing, fixation and staining of slides followed by on-site microscopy. In cases demonstrating inadequate material, the radiologist was advised to repeat the procedure with the aim to achieve sufficient diagnostic material. Thus, the radiologist received immediate feed-back on the specimens achieved and could thereby improve the way of performing FNAB. Furthermore, this approach minimized confusions and enhanced effective communication between radiologist and cytopathologist. At community Hospital A, both the radiologist who performed the majority of the US examinations including FNABs and the technical staff, regularly had the possibility to consult a cytopathologist, thus getting advice concerning technical procedures for making high quality smears, fixation and staining. A cytopathologist appeared only once weekly at the radiology department, and was occupied evaluating specimens from a broad spectre of lesions. She was not able to participate on-site when fine needle aspiration of the thyroid lesions was performed. At community Hospital B, inexperienced technical staff made the smears. All specimens were submitted to our department at NRH for evaluation. No information concerning the potential need for repeat FNAB was available to the radiologist when the FNAB was performed. The need for repeat FNAB implied calling up the patients, including several patients with a long distance to travel from their home to the hospital. In this study we did not aim to decipher which of the mentioned factors that contributed the most to the substantial difference in percentage of unsatisfactory specimens at community Hospital B compared to NRH and community Hospital A.

The national and international guidelines for management of patients with thyroid nodules and differentiated thyroid cancer strongly recommends that thyroid ultrasound should be 
performed in all patients with known or suspected thyroid nodules, and that ultrasound should be used to guide when performing FNAB $(90 ; 91 ; 172)$. In nodules with cystic changes, fibrosis and/or calcifications, US-guidance can clearly target the needle to the cellular/solid portion of the lesion to retrieve diagnostic material. In one study of prevalence and distribution of carcinoma in patients with solitary and multiple thyroid nodules on sonography, the likelihood of thyroid cancer per patient was found to be independent of the number of nodules, underscoring the importance of US-evaluation as a guidance for the FNAB (22). Some studies state that on-site presence of a cytopathologist or cytotechnologist to immediately assess specimen adequacy and representativeness may decrease the number of needle passes $(94 ; 173)$. Using combined US-guided FNAB and on-site evaluation, rates of inadequate samples are reported to be low, varying from $2.8 \%$ to $4 \%(174 ; 175)$. On-site evaluation of FNAB smears may be responsible for cost savings avoiding repeated aspirates. Indirect socioeconomically cost savings include reduced time off work, and shorter hospital stays. Furthermore, when the on-site evaluation reveals a diagnosis of thyroid carcinoma, a more thorough US examination of the neck for lymph nodes may contribute to an efficient preoperative management before operative procedure.

Concerning the technical aspects of the FNAB-procedure, studies have demonstrated that the use of needles of 25-27-gauge and non aspiration method (Zajdela-technique) (176) minimizes pain and reduces blood contamination (94). Some clinicians suggest, in a recent study, that the use of a stylet needle may limit the amount of blood flowing into the needle and thus improve the diagnostic yield $(177 ; 178)$. In a study by Redman et al $(96)$, the inadequacy rate varied significantly among physicians, being lowest (3\%) when pathologists and endocrinologists at teaching hospitals co-operated using US-guided FNAB. The 
inadequacy rate was highest when FNAB was performed by surgeons (18\%) and community physicians (6\%) without US guidance.

\section{Paper II}

This study demonstrates the utility of using Tg-FNAB measurement in combination with cytology to reveal metastases from PTC to neck lymph nodes. The measurement of Tg-FNAB was proved to be particularly useful in cases with cystic metastases and in cases with indeterminate cytology.

The usefulness of thyroglobulin detection in FNAB from neck lesions was described by Pacini and co workers almost 20 years ago (99). However, except for one paper by Frasoldati et al 1999 (162), the usefulness of this method in diagnostics is barely described in the literature before 2003. The multidisciplinary team at the Norwegian Radium Hospital introduced measurement of Tg-FNAB as early as March 2004. Due to the uplifting experience and results, the method was 2006 implemented as a routine in the diagnostic procedure of diagnosing neck lymph nodes at our hospital. This implementation is in line with international guidelines. The European Thyroid Association (90) recommends use of the FNA thyroglobulin assay to confirm suspected lymph node metastases. By contrast, in the American Thyroid Association guidelines (127), for the work-up of patients with differentiated PTC, measurement of thyroglobulin levels in FNA wash-out fluid is not even considered. The lack of consensus possibly reflects the fact that the FNA thyroglobulin assay has never been standardized. As described in Paper II, Tg-FNAB measurement should always be compared with Tg values in serum. In the years following our report several other groups have confirmed our results, supporting the usefulness of Tg-FNAB in diagnosis of differentiated thyroid carcinoma nodal metastases (179-186). 
Tg-FNAB positive results must, for several reasons, be interpreted cautiously. Not all pathological lesions on the neck are lymph node metastases. And not even when these lesions are Tg-FNAB positive, can we make a diagnosis of malignancy. Additionally, the finding of epithelial cells suggestive of follicular epithelium in smears from a neck lesion outside the thyroid bed has to be interpreted cautiously. The demonstration of a mixture of lymphoid cells and follicular cells in smears from a neck lesion outside the thyroid gland, does not always implicate metastasis from PTC. In the central compartment of the neck there is a possibility of a thyreoglossal cyst and in the lateral compartments the finding could be consistent with aberrant benign thyroid parenchyma. The finding of a mixture of lymphoid cells and follicular cells with irregular nuclei in neck nodules outside the thyroid gland in patients shown to have extensive Hashimoto thyroiditis is, to our experience, a diagnostic pitfall. This underscores the importance of adequate clinical information and a close collaboration between the clinician, the radiologist and the cytopathologist.

Of the samples in our study investigated before thyroidectomi, two samples had false positive Tg-FNAB; one patient with differentiated thyroid carcinoma (but no neck lymph node metastasis was found after histological examination) and one patient with benign thyroid disease. We do not know the reason for this false positive Tg-FNAB. In patients who have the whole or part of the thyroid gland intact, Tg leakage into the lymph may occur as a consequence of clinical or ultrasonographical examination, causing detectable $\mathrm{Tg}$ in lymph node FNAB wash-out specimens. Theoretically the patients could carry a DTC micrometastasis, and in the case of benign thyroid disease, the primary tumor might have been so tiny that it was found neither at gross examination nor at microscopy. In both cases the patient was followed. No malignant tumor has so far been verified. 
The interference of $\mathrm{TgAb}$ when measuring serum $\mathrm{Tg}$ may be a technical problem, comprising the use of serum $\mathrm{Tg}$ as a tumor marker test for DTC patients with $\operatorname{TgAb}$ (163). Boi et al assayed $\mathrm{TgAb}$ together with $\mathrm{Tg}$ in FNAB wash-out specimen in eight patients with positive serum $\mathrm{TgAb}$, and showed that $\mathrm{TgAb}$ were detected in FNAB washout fluid in two of these patients. Tg-FNAB concentration were found to be lower in these two specimens than in those found in $\mathrm{TgAb}$ negative FNAB wash-out specimens. The concentration of Tg-FNAB remained, however, clearly above cutoff levels even in the presence of detecable $\operatorname{TgAb}$ in FNAB washout specimens. We did not find $\mathrm{TgAb}$ in the wash-out specimens, and consequently, we interference with $\mathrm{TgAb}$ was not a problem in our samples.

When demonstrating Tg in wash-out from lymph nodes in the central neck department in the case of diagnosed PTC, surgery will automatically include resection of central neck lymph nodes. However, a comprehensive bilateral resection of the central neck compartment lymph nodes may have been performed in any case. In contrast, resection of lymph nodes in the lateral neck compartment is seldom performed prophylactic. When metastasis to lymph nodes in the lateral neck compartment is revealed, by FNAB or Tg-FNAB, or findings are suggestive for metastasis in the lateral compartment, the surgery will become much more extensive, implying a risk for higher morbidity. When no primary carcinoma is revealed in the thyroid gland, a positive Tg-FNAB must be interpreted cautiously. The manipulation of the thyroid gland when examining the neck may, as earlier mentioned, cause a detectable Tg-FNAB. Furthermore, when performing the FNAB on lymph nodes located in the central neck compartments, the needle may be contaminated with Tg while passing through normal thyroid parenchyma. When S-Tg is high, contamination with blood in the FNAB may give a detectable Tg-FNAB. To avoid unnecessary thyroidectomies in cases displaying detecable 
Tg-FNAB caused by contamination, all candidate cases for surgery should be discussed in a multidisciplinary meeting. The absence of radiological findings suspicious of thyroid cancer combined with a negative cytology from the lymph node examined may prevent surgical intervention in anticipation of a more extensive investigation.

\section{Paper III}

This study has shown that US-guided PEI treatment of metastatic lymph nodes may be an excellent alternative to surgery in patients with a limited number of neck metastases from PTC.

Percutaneous ethanol injection has been in use for treating certain types of tumors (parathyroid adenomas, metastatic liver lesions, small hepatocellular carcinomas and other benign endocrine neoplasms) for almost 30 years (187-189) (190-192). Furthermore, PEI has been shown to represent an effective alternative to surgery and RAI treatment of autonomous functioning thyroid nodules (193-195)and has also been proposed for the treatment of cold benign thyroid nodules $(196 ; 197)$ and thyroid cysts $(198-200)$.

In 2002, Lewis and co workers (140) presented a study which concluded that sonographically guided percutaneous ethanol injection is a valuable treatment option for patients with limited cervical nodal metastases from PTC. We established the PEI procedure as treatment for cervical nodal metastases at NRH in June 2004. The treatment has since then been in use in the everyday practice at an out-patient clinic at the NRH. As far as we are informed, no other hospitals in Norway or even in Europe are practicing PEI treatment routinely of neck lymph node metastases from PTC. As being one of few hospitals practicing PEI treatment of metastatic neck lymph nodes, our study was acknowledged in an editorial by Ian D. Hay and 
J. William Charboneau at Mayo Clinic in the same issue of JECM publishing our manuscript (201). At NRH the PEI treatment has even been given to a few patients with local recurrence of PTC, unsuitable for surgery and lacking response to RAI-treatment. The recurrence of tumor in these patients has caused compressive symptoms, and the administration of ethanol has managed to shrink the lesions with transient relief of the symptoms. The treatment has been repeated several times due to recurrent growth. According to these up-lifting results, the criteria for the use of PEI treatment may widen, and foci of recurrent papillary carcinoma inaccessible to surgery can be a target for PEI treatment. Additionally, small foci of primary PTC in patients refusing surgery may be treated with PEI.

Preliminary, rapid, on-site cytologic diagnosis made it possibel to give PEI treatment immediately. This increases the safety of the procedure as well as optimizing utilization of procedure rooms and imaging facilities. Whereas no high-level evidence exists to define whether the addition of central neck lymph node dissection (CLND) to total thyroidectomy for PTC confers greater risk of permanent hypoparathyroidism or permanent nerve injury (132), reoperation in the central neck compartment for recurrent PTC has a considerable risk for permanent injury to the recurrent laryngeal nerve and the parathyroid glands than initial total thyroidectomy with or without CLND (202). The incidence of temporary hypoparathyroidism ranges from $0.3 \%$ to $13 \%$ in several large series $(137 ; 203 ; 204)$. Permanent hypoparathyroidism is a devastating and disabling complication that can occur with reoperative thyroid surgery with a reported incidence of $0 \%$ to $3.5 \%$ (137) (138).

All patients included in our study received at least one RAI-treatment after surgery. Metastatic lymph nodes were diagnosed after surgery and RAI-treatment. Pathological lymph nodes were 
detected when performing US examination at follow-up and PTC metastasis was verified with cytomorphology and/or positive Tg-FNAB.

The benefits of surgery (reoperation) and RAI-treatment of patients with differentiated thyroid carcinoma might be outweighed by the risk of complications, which have initiated the search for an alternative treatment of local neck recurrence of PTC. The interest in finding an alternative treatment is driven by the fact that some PTCs are unresponsive to RAI. The decision whether to give RAI-treatment or not can be difficult because of conflicting reports of RAI treatment and medical uncertainty due to the lack of long-term randomized controlled trials (205). Postoperative RAI-treatment can be avoided in low-risk patients and should be individualized. The RAI-treatment is expensive and this approach may reduce the costs.

In our study PEI treatment was cost-effective, efficient, and cheap and could also be performed at an out-patient basis. No follow-up report after long observation period has been performed after PEI treatment of neck lymph node metastases from PTC, but short- and intermediate time follow-up has not revealed any serious side-effects. When performing PEI, the cost of cytological examination of neck lesion with US guidance based on Dutch Health Care Tariffs (2010) is $€ 232$ (206) and the additional cost for the tiny amount of ethanol given is diminutive.

\section{Paper IV}

Thus, no new procedures have been implemented as a result of the study of the markers DDIT3, STT3A, ARG2 and FAM129A. We have been reminded of the fact that no "magic tumor marker" able to differentiate between benign and malignant follicular tumor can be 
included in the established immunohistochemical panel without being verified by new independent observations. In our study, evaluation of the histological slides was performed independently by two pathologists. All discordant cases were re-evaluated using a multiheaded microscope, and consensus was always achieved. Discrepancy in the diagnosis when reviewing thyroid specimen is frequent, and was found to be $18 \%$ in one study by Hamady et al (208). Since major therapeutic and prognostic modifications may be adjusted according to a second histological opinion, cases of suspected thyroid cancers should be reviewed in a multidisciplinary meeting, and this has been done at NRH.

In molecular studies large numbers of people have been screened for thousands of genes with the aim to identify diagnostic and prognostic markers for thyroid cancer. When considering potential new diagnostic markers, a well known problem is that the decision relies on published studies and can only incompletely identify possible biases in them. There is a bias in the fact that when a study presents "negative results", it may not be submitted or accepted for publication. Journal editors should have an obligation to publish negative results. In many cases extensive effort has been performed to replicate the results in published works. When critical evidence provide that differences in reagents and methods are not responsible for the failure of other replication studies, these new studies should be published with the same matter of course. Other researchers provided with the data will be informed, so that if they want to investigate the same area, they can try alternatives or modifications rather than simply repeat results that were unpublished. Another aspect is described in an editorial by Pfeffer et al (207): "If the "negative results" are the consequence of some fundamental flaw in methods that are commonly used, further analysis by others may help uncover those flaws and lead to methodological improvement". Improper use of immunologic tests can result in misdiagnosis, inappropriate therapy, and wasted health care resources. 
The morphology has been, and will still be the gold standard. New techniques are often expensive and time-consuming. It will be an imperative that the new diagnostic techniques must provide information of prognostic or therapeutic significance beyond that provided by the current gold standard. Large multicenter biomarker studies are advisable for identification of novel biomarkers that may have clinical utility for thyroid cancer diagnosis. In these studies large patient cohorts are required for both the development and the validation of a test. Robust predictors may be identified through using multiple classification algorithms to select genes. For validation test IHC on TMAs may be useful enabling potentially new biomarkers to be tested on a large number of tumor samples. Validations tests should also be performed on a set of samples matched for different clinically variables. Comparison of study results can be strengthened through standardization of IHC technique and criteria for marker interpretation.

\section{Methodological Considerations (Paper II-IV)}

A sensitive in-house time-resolved fluoroimmunoassay previously established in the Department of Medical Biochemistry at Oslo University Hospital, described in Paper II, was chosen for the measurements of Tg-FNAB (Paper II and III). The specificity of immunoassay depends, as earlier mentioned, on the degree of a specific antibody to bind to an epitope (antigen). The monoclonal antibodies we used have high affinity for $\mathrm{Tg}$. To reduce interference from autoantibodies $(\mathrm{TgAb})$ the hybridomas were selected in the presence of autoantibodies. The Tg assay was also less vulnerable to interference of heterofilic antibodies due to modification of the solid phase mAb. The assay we used was standardized against a reference preparation. When performing the wash-out procedure rinsing the needle in the matrix buffer or different amounts of saline, the amount of specimen was variable, and the $\mathrm{Tg}$ values were evaluated without considering dilution. In contrast to several other studies 
$(181 ; 183 ; 209)$, we did not define a definite cut-off value for Tg-FNAB, as we consider it to be important to evaluate the results of the measurements of Tg-FNAB with regard to S-Tg and S$\operatorname{TgAb}$. The manipulation of the thyroid gland when examining the neck may cause false positive Tg measurements. Thus the diagnostic significance of Tg-FNAB differs in patients with or without thyroid gland which should also be taken into consideration when evaluating the values of Tg-FNAB measurements.

Immunohistochemical studies (Paper IV) of diagnostic markers should ideally follow guidelines for standardization to guarantee better methodological quality. According to Sanabria et al (102) diagnostic test studies should satisfy several conditions in order to yield reliable results; the study must be prospective, and the study must examine a clinically relevant population, the study must have a gold standard, the evaluation of the new test must be independent and blind, intraobserver and interobserver agreement must be assessed and in cases with multiple cutoff points, a stratified analysis with each cutoff point should be tried. The staining protocols and more consistent cutoff points of intensity or frequency of positive tumor cells remain to be standardized for many immunologic markers. The evaluation of immunohistochemical staining is subjective. Several grading systems for evaluation have been developed. Some are considering the staining of the nuclei, the cytoplasm and the cell membrane separately. Others are trying to quantify the intensity of the staining. To be able to compare our evaluation of molecular markers (Paper IV) with the results presented by Cerutti el al $(168 ; 169)$, we chose to perform the immunostaining using the same protocol as Cerutti et al (168). Through this, the risk that the differences in results could be explained by differing methodologies was minimized. No standardization of the evaluation of the four immunomarkers investigated has been established. The reproducibility of the evaluation is diminishing with rising numbers of parameters subjectively assessed. Cerutti et al (168) 
evaluated staining both in the nucleus and the cytoplasm, but they did not define a cut-off for the fraction of positive cells needed to categorize the case as positive. When examining the immunohistochemical slides, we found cytoplasmic immunostaining to be the most appropriate. We did not find cases showing positive staining only in the nuclei. The immunohistochemical slides were scored in a semiquantitative manner as was performed by Cerutti et al (168). Additionally, we used a predetermined cutoff of $20 \%(<20 \%$ tumor cells staining $=$ negative, $\geq 20 \%=$ positive) to unequivocally categorize cases in two groups when calculating sensitivity and specificity. The interobserver agreement was assessed and was satisfactory. 


\section{Conclusions}

\section{General}

We demonstrated that experienced and skilled personnel increase the accuracy of FNAB specimens. Furthermore new reliable procedures for follow-up of patients with DTC were established.

\section{Specific}

1. The fraction of representative and diagnostic FNAB specimens from thyroid lesions was higher in hospitals where dedicated and skilled physicians participated in the ultrasound guided FNAB procedure (Paper I).

2. Tg measurement of needle wash-out specimens from suspicious neck lymph nodes increased the detection rate of metastases from PTC. This was particularly the case for cystic metastases (Paper II).

3. PEI of neck lymph node metastases from PTC was efficient, cheap and without side effects. The efficacy of the PEI treatment was confirmed by US examination (disappearance or reduced size and vascularity of the lymph nodes) and by the combined use of cytomorphology and Tg measurements (no detectable $\mathrm{Tg}$ ) in needle wash-out specimens (Paper III).

4. The four molecular markers DDIT3, ARG2, STT3A and FAM129A were not helpful in separating FTA from FTC (Paper IV). 


\section{Future Perspectives}

The future perspectives for management of patients with thyroid lesions will be dependent on some important assumptions being fulfilled:

1) The engagement, knowledge and skill of all physicians involved

2) The establishment and functionality of a multidisciplinary thyroid team

3) Optimal collaboration between pathologist and bench scientists

4) The electronic journal must allow all relevant clinical information to be registered and be available to the pathologist, and subsequently be included in the report to the clinician.

The role of the Pathologist in future management of patients with thyroid lesions The role of the pathologist in thyroid diagnostics has changed over the last 10 years, and might change even more in the near future. This can be seen in the light of the three following fields: Personalized Medicine, Informatics and Multidisciplinary groups. In all these areas the thyroid pathologists are, or should be, heavily involved due to their highly specific knowledge of anatomic pathology, tumor biology and management.

\section{Personalized Medicine}

Personalized medicine is sometimes referred to as personalized health care that according to the Personalized Health Care Expert Panel Meeting 2007 (210) is described as follows: "Personalized health care draws molecular- and cellular-level disease processes, health states, behavioural and environmental determinants and response to interventions. It applies this to deliver patient-specific health care that reflects individual risks and benefits of particular 
treatments, to determine risks of particular conditions or diseases and to facilitate the discovery and validation of health care products and other interventions".

The impact of personalized medicine on the management of thyroid disorders is growing. Like malignant tumors originating in other organs, the management of lesions in the thyroid gland should be individualized according to both the patient's and the nodules characteristics. Some describe this as the dawn of the era of personalized thyroid medicine, and the end of the era of "one fits all" medicine. The knowledge of genetic, epigenetic and molecular biomarkers of thyroid tumors rise among scientists, and the physicians participating in multidisciplinary thyroid teams will need to be informed on how to utilize this new information in the care of their patients. It is important not only to give the thyroid patients the best treatment, but also to avoid using time and money on markers and treatment that do not work. The pathologists may act as a bridge between biomedical researchers and clinicians. The pathologist will be extensively involved in three important aspects of personalized medicine; the development and validation of novel biochemical and molecular markers, the quality assurance of cytological specimens, and the establishment of genetic banking systems. It is of crucial importance that the pathologist examines the morphology of all specimens included in studies using tissue samples for investigation of molecular markers. Both paraffin embedded samples and fresh specimens might consist of cancer tissue as well as benign parenchyma. Microdissection enabling the investigator to isolate tumor tissue from the benign surrounding parenchyma will in many cases be necessary.

The development in management of patients with thyroid tumors made the American Thyroid Association (ATA) to choose Personalized Medicine as their theme for their recent spring (2010) symposium "Thyroid Disorders in the Era of Personalized Medicine" (211). 


\section{Informatics in Practical Pathology}

Pathology informatics may be defined as the discipline and practice of using electronic information systems to facilitate, promote, and advance the practice of pathology, as exemplified in pathology's tripartite mission of patient care, physician education, and the understanding of disease (15). An enormous amount of studies presenting molecular analysis of thyroid tumors have been published. Some of the alterations discovered and characterized have significant diagnostic utility, and some may serve as potential therapeutic targets. As mentioned earlier, the molecular marker BRAF, i.e., offer help in risk stratification and can be potentially used to optimize surgical and postsurgical management of patients with thyroid cancer. The genetic markers developed for use in diagnostics are, however, not the only advances in thyroid pathology. The documentation of digital images make it easy to compare different samples and to consult outside experts, the availability of up-dated classification and information on-line, the immediate access to clinical and radiological reports, are all improvements for management of patients with thyroid lesions.

\section{The role of multidisciplinary groups in managing thyroid lesions}

A multidisciplinary team is, according to the UK Manual for Cancer Services 2004 (212), defined as "a group of people of different healthcare disciplines, which meets together at a given time (whether physically in one place, or by video or teleconferencing) to discuss a given patient and who are each able to contribute independently to the diagnostic and treatment decisions about the patient". The advantages of a multidisciplinary team looking after thyroid cancer are well known and have been detailed in the Manual for Improving Outcomes in Head and Neck Cancer by the National Institute of Clinical Excellence (213). In a review concerning new therapeutic approaches for metastatic thyroid carcinoma in Lancet Oncology 2007, E. Baudin and M. Sclumberger conclude "that to increase the accrual of 
patients to trials, optimise the experimental design of the protocol, improve the characterisation of tumor tissues, and improve the tolerance of treatment, the participation of endocrinologists, oncologists, specialists of nuclear medicine, surgeons, pathologist, researchers, and statisticians needs to be strongly encouraged" (214).

\section{Molecular predictive markers for use on FNAB specimens}

The knowledge acquired during the recent years about molecular genetics of thyroid cancer has been shown to be especially useful in the preoperative diagnosis of thyroid cancer.

On FNAB specimens the category "Follicular lesion of undetermined significance/atypia of undetermined significance" is defined as being heterogeneous and a class that cannot be classified as benign, suspicious for follicular neoplasm, or suspicious for malignancy according to the new Bethesda 2007 Thyroid Cytology Classification (92). The risk of malignancy in this group has been estimated at 5-10\% and falls between benign (1\%) and follicular neoplasm (20-30\%) diagnoses. Although repeat FNAB may provide clarification, the use of ancillary testing can be helpful in separating cases into a high-risk group and a lowrisk group (215). In a recent prospective study (216), 967 FNAB samples were analyzed for a panel of mutations consisting of BRAF, NRAS, HRAS, KRAS point mutations and RET/PTC1, RET/PTC3 and PAX8/PPAR $\gamma$ rearrangements. Eighty-seven mutations were revealed including $19 \mathrm{BRAF}, 62 \mathrm{RAS}, 1 \mathrm{RET} / \mathrm{PTC}$, and five PAX8/PPAR $\gamma$. BRAF, RET/PTC and PAX8/PPAR $\gamma$ mutations were associated with malignancy in close to $100 \%$ of nodules. The negative predictive value of molecular testing indicated that the cancer risk in mutation-negative nodules with follicular neoplasm/suspicious for follicular neoplasm and suspicious for malignant cells was $14 \%$ and 28\%, respectively. American Thyroid Association guidelines recommend diagnostic lobectomy for mutation-negative samples displaying follicular neoplasm/suspicious for follicular neoplasm on cytology (91). 
Testing for point mutations on FNAB specimens, as of BRAF and RAS, can be achieved by a variety of molecular techniques, including probe-specific real-time PCR amplification, realtime allele-specific PCR, direct sequencing, and colorimetric assay (121;217-220). The detection of chromosomal rearrangements, such as RET/PTC and PAX8/PPAR $\gamma$, on freshly collected or snap-frozen FNAB specimen can be reliably performed by reverse-transcriptase in a conventional or real-time mode. Nikiforov and co-workers (216) found that more than $90 \%$ of the residual aspirated material (wash-out specimen) collected during routine FNAB procedure was sufficient for mutation detection.

The number of incidentally diagnosed thyroid nodules will most probably increase. Thus, accurate markers that have a high sensitivity and specificity will be needed to make a significant impact on the management of thyroid lesions. Future clinical trials must, however, be conducted in a way that enables evaluation of the emerging biomarkers and the costeffectiveness of using these biomarkers routinely or selectively in management of thyroid neoplasms.

The pathologists as a diagnostic specialist will in the future play an important role in adopting new diagnostic and prognostic modalities as well as in the use of informatics tools as they become available. 


\section{References}

(1) Masson P. Human Tumors. Detroit: Wayne State University Press: 1970.

(2) Veith FJ, Brooks JR, Grigsby WP, Selenkow HA. The Nodular Thyroid Gland and Cancer. A Practical Approach to the Problem. N Engl J Med 1964 February 27;270:431-6.

(3) Ezzat S, Sarti DA, Cain DR, Braunstein GD. Thyroid incidentalomas. Prevalence by palpation and ultrasonography. Arch Intern Med 1994 August 22;154(16):1838-40.

(4) Hegedus L. Clinical practice. The thyroid nodule. N Engl J Med 2004 October 21;351(17):1764-71.

(5) Lin JD, Chao TC, Huang BY, Chen ST, Chang HY, Hsueh C. Thyroid cancer in the thyroid nodules evaluated by ultrasonography and fine-needle aspiration cytology. Thyroid 2005 July;15(7):708-17.

(6) Yang J, Schnadig V, Logrono R, Wasserman PG. Fine-needle aspiration of thyroid nodules: a study of 4703 patients with histologic and clinical correlations. Cancer 2007 October 25;111(5):306-15.

(7) Leenhardt L, Hejblum G, Franc B, Fediaevsky LD, Delbot T, Le GD et al. Indications and limits of ultrasound-guided cytology in the management of nonpalpable thyroid nodules. J Clin Endocrinol Metab 1999 January;84(1):24-8.

(8) Castro MR, Gharib H. Thyroid fine-needle aspiration biopsy: progress, practice, and pitfalls. Endocr Pract 2003 March;9(2):128-36.

(9) Cappelli C, Tironi A, Mattanza C, Cumetti D, Agosti B, Gandossi E et al. Cost-effectiveness of fineneedle-aspiration cytology of thyroid nodules with intranodular vascular pattern using two different needle types. Endocr Pathol 2005;16(4):349-54.

(10) Baloch ZW, Fleisher S, Livolsi VA, Gupta PK. Diagnosis of "follicular neoplasm": a gray zone in thyroid fine-needle aspiration cytology. Diagn Cytopathol 2002 January;26(1):41-4.

(11) Yeh MW, Demircan O, Ituarte P, Clark OH. False-negative fine-needle aspiration cytology results delay treatment and adversely affect outcome in patients with thyroid carcinoma. Thyroid 2004 March;14(3):207-15.

(12) Machens A, Holzhausen HJ, Dralle H. The prognostic value of primary tumor size in papillary and follicular thyroid carcinoma. Cancer 2005 June 1;103(11):2269-73.

(13) Shibru D, Chung KW, Kebebew E. Recent developments in the clinical application of thyroid cancer biomarkers. Curr Opin Oncol 2008 January;20(1):13-8.

(14) Nikiforov YE. Molecular analysis of thyroid tumors. Mod Pathol 2011 April;24 Suppl 2:S34-S43.

(15) Sinard JH, Morrow JS. Informatics and anatomic pathology: meeting challenges and charting the future. Hum Pathol 2001 February;32(2):143-8.

(16) Clark O, Quan-Yang D, Kebebew E, eds. Textbook of Endocrine Surgery. Philadelphia, PA. Elsevier Saunders. 2005.

(17) Brander A, Viikinkoski P, Nickels J, Kivisaari L. Thyroid gland: US screening in a random adult population. Radiology 1991 December;181(3):683-7.

(18) Mortensen JD, Woolner LB, Bennett WA. Gross and microscopic findings in clinically normal thyroid glands. J Clin Endocrinol Metab 1955 October;15(10):1270-80.

(19) Bisi H, Fernandes VS, de Camargo RY, Koch L, Abdo AH, de BT. The prevalence of unsuspected thyroid pathology in 300 sequential autopsies, with special reference to the incidental carcinoma. Cancer 1989 November 1;64(9):1888-93.

(20) De Lellis RA, Lloyd RV, Heitz PU, Eng C. World Health Organization Classification of tumours. Pathology and genetics of tumours of endocrine organs. Lyon, France: IARC Press; 2004.

(21) Surveillance Epidemiology and End Results. National Cancer Institute. 2011. http://seer.cancer.gov/statfacts/html/thyro.html\#risk

(22) Frates MC, Benson CB, Doubilet PM, Kunreuther E, Contreras M, Cibas ES et al. Prevalence and distribution of carcinoma in patients with solitary and multiple thyroid nodules on sonography. $\mathrm{J}$ Clin Endocrinol Metab 2006 September;91(9):3411-7.

(23) Cancer Registry of Norway. 2009. http://www.kreftregisteret.no/en/

(24) Davies L, Welch HG. Increasing incidence of thyroid cancer in the United States, 1973-2002. JAMA 2006 May 10;295(18):2164-7.

(25) How J, Tabah R. Explaining the increasing incidence of differentiated thyroid cancer. CMAJ 2007 November 20;177(11):1383-4.

(26) Ron E, Lubin JH, Shore RE, Mabuchi K, Modan B, Pottern LM et al. Thyroid cancer after exposure to external radiation: a pooled analysis of seven studies. Radiat Res 1995 March;141(3):259-77. 
(27) Moysich KB, Menezes RJ, Michalek AM. Chernobyl-related ionising radiation exposure and cancer risk: an epidemiological review. Lancet Oncol 2002 May;3(5):269-79.

(28) Cardis E, Kesminiene A, Ivanov V, Malakhova I, Shibata Y, Khrouch V et al. Risk of thyroid cancer after exposure to 131I in childhood. J Natl Cancer Inst 2005 May 18;97(10):724-32.

(29) Hemminki K, Eng C, Chen B. Familial risks for nonmedullary thyroid cancer. J Clin Endocrinol Metab 2005 October;90(10):5747-53.

(30) Nikiforov YE. RET/PTC rearrangement in thyroid tumors. Endocr Pathol 2002;13(1):3-16.

(31) Soares P, Trovisco V, Rocha AS, Lima J, Castro P, Preto A et al. BRAF mutations and RET/PTC rearrangements are alternative events in the etiopathogenesis of PTC. Oncogene 2003 July 17;22(29):4578-80.

(32) Kimura ET, Nikiforova MN, Zhu Z, Knauf JA, Nikiforov YE, Fagin JA. High prevalence of BRAF mutations in thyroid cancer: genetic evidence for constitutive activation of the RET/PTC-RAS-BRAF signaling pathway in papillary thyroid carcinoma. Cancer Res 2003 April 1;63(7):1454-7.

(33) Nikiforov YE, Nikiforova MN. Molecular genetics and diagnosis of thyroid cancer. Nat Rev Endocrinol 2011 October;7(10):569-80.

(34) Nose V. Familial thyroid cancer: a review. Mod Pathol 2011 April;24 Suppl 2:S19-S33.

(35) Kameyama K, Takami H, Miyajima K, Mimura T, Hosoda Y, Ito K et al. Papillary carcinoma occurring within an adenomatous goiter of the thyroid gland in Cowden's disease. Endocr Pathol 2001;12(1):73-6.

(36) Nikiforova MN, Nikiforov YE. Molecular genetics of thyroid cancer: implications for diagnosis, treatment and prognosis. Expert Rev Mol Diagn 2008 January;8(1):83-95.

(37) Nagataki S, Nystrom E. Epidemiology and primary prevention of thyroid cancer. Thyroid 2002 October;12(10):889-96.

(38) Balkwill F, Mantovani A. Inflammation and cancer: back to Virchow? Lancet 2001 February 17;357(9255):539-45.

(39) Kacinski BM. CSF-1 and its receptor in ovarian, endometrial and breast cancer. Ann Med 1995 February;27(1):79-85.

(40) Obermueller E, Vosseler S, Fusenig NE, Mueller MM. Cooperative autocrine and paracrine functions of granulocyte colony-stimulating factor and granulocyte-macrophage colony-stimulating factor in the progression of skin carcinoma cells. Cancer Res 2004 November 1;64(21):7801-12.

(41) DeClerck YA, Mercurio AM, Stack MS, Chapman HA, Zutter MM, Muschel RJ et al. Proteases, extracellular matrix, and cancer: a workshop of the path B study section. Am J Pathol 2004 April;164(4):1131-9.

(42) Carmeliet P, Jain RK. Angiogenesis in cancer and other diseases. Nature 2000 September 14;407(6801):249-57.

(43) Soh EY, Duh QY, Sobhi SA, Young DM, Epstein HD, Wong MG et al. Vascular endothelial growth factor expression is higher in differentiated thyroid cancer than in normal or benign thyroid. J Clin Endocrinol Metab 1997 November;82(11):3741-7.

(44) Klein M, Picard E, Vignaud JM, Marie B, Bresler L, Toussaint B et al. Vascular endothelial growth factor gene and protein: strong expression in thyroiditis and thyroid carcinoma. J Endocrinol 1999 April;161(1):41-9.

(45) Kato S, Kobayashi T, Yamada K, Nishii K, Sawada H, Ishiguro H et al. Expression of erbB receptors mRNA in thyroid tissues. Biochim Biophys Acta 2004 August 4;1673(3):194-200.

(46) Schiff BA, McMurphy AB, Jasser SA, Younes MN, Doan D, Yigitbasi OG et al. Epidermal growth factor receptor (EGFR) is overexpressed in anaplastic thyroid cancer, and the EGFR inhibitor gefitinib inhibits the growth of anaplastic thyroid cancer. Clin Cancer Res 2004 December 15;10(24):8594-602.

(47) Di Pasquale M, Rothstein JL, Palazzo JP. Pathologic features of Hashimoto's-associated papillary thyroid carcinomas. Hum Pathol 2001 January;32(1):24-30.

(48) Franceschi S, Preston-Martin S, Dal ML, Negri E, La VC, Mack WJ et al. A pooled analysis of casecontrol studies of thyroid cancer. IV. Benign thyroid diseases. Cancer Causes Control 1999 December;10(6):583-95.

(49) Marques AR, Espadinha C, Catarino AL, Moniz S, Pereira T, Sobrinho LG et al. Expression of PAX8PPAR gamma 1 rearrangements in both follicular thyroid carcinomas and adenomas. J Clin Endocrinol Metab 2002 August;87(8):3947-52.

(50) Cerci C, Cerci SS, Eroglu E, Dede M, Kapucuoglu N, Yildiz M et al. Thyroid cancer in toxic and nontoxic multinodular goiter. J Postgrad Med 2007 July;53(3):157-60.

(51) Feldt-Rasmussen U. Iodine and cancer. Thyroid 2001 May;11(5):483-6.

(52) Knudsen N, Laurberg P, Perrild H, Bulow I, Ovesen L, Jorgensen T. Risk factors for goiter and thyroid nodules. Thyroid 2002 October;12(10):879-88.

(53) Delange F, Lecomte P. Iodine supplementation: benefits outweigh risks. Drug Saf 2000 February;22(2):89-95. 
(54) Weissel M. Legal augmentation of iodine content in table salt from 10 to $20 \mathrm{mg} \mathrm{KI} / \mathrm{kg}$ : documented effects a decade later. Exp Clin Endocrinol Diabetes 2003 June;111(4):187-90.

(55) Guan H, Ji M, Bao R, Yu H, Wang Y, Hou P et al. Association of high iodine intake with the T1799A BRAF mutation in papillary thyroid cancer. J Clin Endocrinol Metab 2009 May;94(5):1612-7.

(56) Verkooijen HM, Fioretta G, Pache JC, Franceschi S, Raymond L, Schubert H et al. Diagnostic changes as a reason for the increase in papillary thyroid cancer incidence in Geneva, Switzerland. Cancer Causes Control 2003 February; 14(1):13-7.

(57) Paget S. The distribution of secondary growths in cancer of the breast. 1889. Cancer Metastasis Rev 1989 August;8(2):98-101.

(58) Mueller MM, Fusenig NE. Friends or foes - bipolar effects of the tumour stroma in cancer. Nat Rev Cancer 2004 November;4(11):839-49.

(59) Kalluri R, Zeisberg M. Fibroblasts in cancer. Nat Rev Cancer 2006 May;6(5):392-401.

(60) Thiery JP, Acloque H, Huang RY, Nieto MA. Epithelial-mesenchymal transitions in development and disease. Cell 2009 November 25;139(5):871-90.

(61) Chaffer CL, Weinberg RA. A perspective on cancer cell metastasis. Science 2011 March 25;331(6024):1559-64.

(62) Folkman J. Fundamental concepts of the angiogenic process. Curr Mol Med 2003 November;3(7):64351.

(63) Thiery JP, Sleeman JP. Complex networks orchestrate epithelial-mesenchymal transitions. Nat Rev Mol Cell Biol 2006 February;7(2):131-42.

(64) Wyckoff JB, Wang Y, Lin EY, Li JF, Goswami S, Stanley ER et al. Direct visualization of macrophageassisted tumor cell intravasation in mammary tumors. Cancer Res 2007 March 15;67(6):2649-56.

(65) Prall F. Tumour budding in colorectal carcinoma. Histopathology 2007 January;50(1):151-62.

(66) Sleeman JP. The lymph node as a bridgehead in the metastatic dissemination of tumors. Recent Results Cancer Res 2000;157:55-81.

(67) Vasko V, Espinosa AV, Scouten W, He H, Auer H, Liyanarachchi S et al. Gene expression and functional evidence of epithelial-to-mesenchymal transition in papillary thyroid carcinoma invasion. Proc Natl Acad Sci U S A 2007 February 20;104(8):2803-8.

(68) Scheumman GF, Hoang-Vu C, Cetin Y, Gimm O, Behrends J, Von WR et al. Clinical significance of Ecadherin as a prognostic marker in thyroid carcinomas. J Clin Endocrinol Metab 1995 July;80(7):216872 .

(69) Kato N, Tsuchiya T, Tamura G, Motoyama T. E-cadherin expression in follicular carcinoma of the thyroid. Pathol Int 2002 January;52(1):13-8.

(70) Liu Z, Kakudo K, Bai Y, Li Y, Ozaki T, Miyauchi A et al. Loss of cellular polarity/cohesiveness in the invasive front of papillary thyroid carcinoma, a novel predictor for lymph node metastasis; possible morphological indicator of epithelial mesenchymal transition. J Clin Pathol 2011 April;64(4):325-9.

(71) Bai Y, Kakudo K, Nakamura M, Ozaki T, Li Y, Liu Z et al. Loss of cellular polarity/cohesiveness in the invasive front of papillary thyroid carcinoma and periostin expression. Cancer Lett 2009 August 28;281(2):188-95.

(72) Prazeres H, Torres J, Rodrigues F, Pinto M, Pastoriza MC, Gomes D et al. Chromosomal, epigenetic and microRNA-mediated inactivation of LRP1B, a modulator of the extracellular environment of thyroid cancer cells. Oncogene 2011 March 17;30(11):1302-17.

(73) Ramsden JD. Angiogenesis in the thyroid gland. J Endocrinol 2000 September;166(3):475-80.

(74) Turner HE, Harris AL, Melmed S, Wass JA. Angiogenesis in endocrine tumors. Endocr Rev 2003 October;24(5):600-32.

(75) Fellmer PT, Sato K, Tanaka R, Okamoto T, Kato Y, Kobayashi M et al. Vascular endothelial growth factor-C gene expression in papillary and follicular thyroid carcinomas. Surgery 1999 December;126(6):1056-61.

(76) Hung CJ, Ginzinger DG, Zarnegar R, Kanauchi H, Wong MG, Kebebew E et al. Expression of vascular endothelial growth factor-C in benign and malignant thyroid tumors. J Clin Endocrinol Metab 2003 August;88(8):3694-9.

(77) van Heerden JA, Hay ID, Goellner JR, Salomao D, Ebersold JR, Bergstralh EJ et al. Follicular thyroid carcinoma with capsular invasion alone: a nonthreatening malignancy. Surgery 1992 December;112(6):1130-6.

(78) Sipos JA, Mazzaferri EL. Thyroid cancer epidemiology and prognostic variables. Clin Oncol (R Coll Radiol ) 2010 August;22(6):395-404.

(79) Hassan I, Barth P, Celik I, Hoffmann S, Langer P, Ramaswamy A et al. An authentic malignant epithelioid hemangioendothelioma of the thyroid: a case report and review of the literature. Thyroid 2005 December;15(12):1377-81. 
(80) Beer TW. Malignant thyroid haemangioendothelioma in a non-endemic goitrous region, with immunohistochemical evidence of a vascular origin. Histopathology 1992 June;20(6):539-41.

(81) Maiorana A, Collina G, Cesinaro AM, Fano RA, Eusebi V. Epithelioid angiosarcoma of the thyroid. Clinicopathological analysis of seven cases from non-Alpine areas. Virchows Arch 1996 October;429(2-3):131-7.

(82) Proces S, Schroeyers P, Delos M, Michel L, Donckier J. Angiosarcoma of the thyroid and concurrent hyperthyroidism. J Endocrinol Invest 1998 January;21(1):67-9.

(83) Thompson NW, Dunn EL, Batsakis JG, Nishiyama RH. Hurthle cell lesions of the thyroid gland. Surg Gynecol Obstet 1974 October;139(4):555-60.

(84) Asa SL. My approach to oncocytic tumours of the thyroid. J Clin Pathol 2004 March;57(3):225-32.

(85) Silver CE, Owen RP, Rodrigo JP, Rinaldo A, Devaney KO, Ferlito A. Aggressive variants of papillary thyroid carcinoma. Head Neck 2011 July;33(7):1052-9.

(86) Akslen LA, Livolsi VA. Prognostic significance of histologic grading compared with subclassification of papillary thyroid carcinoma. Cancer 2000 April 15;88(8):1902-8.

(87) De Lellis RA. Tumors of the Thyroid Gland, Atlas of Tumor Pathology. Washington DC, USA: Armed Forces Institute of Pathology; 1992.

(88) Hay ID. Management of patients with low-risk papillary thyroid carcinoma. Endocr Pract 2007 September;13(5):521-33.

(89) Mazzaferri EL. Management of low-risk differentiated thyroid cancer. Endocr Pract 2007 September;13(5):498-512.

(90) Pacini F, Schlumberger M, Dralle H, Elisei R, Smit JW, Wiersinga W. European consensus for the management of patients with differentiated thyroid carcinoma of the follicular epithelium. Eur J Endocrinol 2006 June;154(6):787-803.

(91) Cooper DS, Doherty GM, Haugen BR, Kloos RT, Lee SL, Mandel SJ et al. Revised American Thyroid Association management guidelines for patients with thyroid nodules and differentiated thyroid cancer. Thyroid 2009 November;19(11):1167-214.

(92) Cibas ES, Ali SZ. The Bethesda System for Reporting Thyroid Cytopathology. Thyroid 2009 November;19(11):1159-65.

(93) Eedes CR, Wang HH. Cost-effectiveness of immediate specimen adequacy assessment of thyroid fineneedle aspirations. Am J Clin Pathol 2004 January;121(1):64-9.

(94) Ceresini G, Corcione L, Morganti S, Milli B, Bertone L, Prampolini R et al. Ultrasound-guided fineneedle capillary biopsy of thyroid nodules, coupled with on-site cytologic review, improves results. Thyroid 2004 May; 14(5):385-9.

(95) Ghofrani M, Beckman D, Rimm DL. The value of onsite adequacy assessment of thyroid fine-needle aspirations is a function of operator experience. Cancer 2006 April 25;108(2):110-3.

(96) Redman R, Zalaznick H, Mazzaferri EL, Massoll NA. The impact of assessing specimen adequacy and number of needle passes for fine-needle aspiration biopsy of thyroid nodules. Thyroid 2006 January; 16(1):55-60.

(97) Carling T, Long WD, III, Udelsman R. Controversy surrounding the role for routine central lymph node dissection for differentiated thyroid cancer. Curr Opin Oncol 2010 January;22(1):30-4.

(98) Coleman SC, Smith JC, Burkey BB, Day TA, Page RN, Netterville JL. Long-standing lateral neck mass as the initial manifestation of well-differentiated thyroid carcinoma. Laryngoscope 2000 February; 110 (2 Pt 1):204-9.

(99) Pacini F, Fugazzola L, Lippi F, Ceccarelli C, Centoni R, Miccoli P et al. Detection of thyroglobulin in fine needle aspirates of nonthyroidal neck masses: a clue to the diagnosis of metastatic differentiated thyroid cancer. J Clin Endocrinol Metab 1992 June;74(6):1401-4.

(100) Permanetter W, Nathrath WB, Lohrs U. Immunohistochemical analysis of thyroglobulin and keratin in benign and malignant thyroid tumours. Virchows Arch A Pathol Anat Histopathol 1982;398(2):221-8.

(101) de Matos PS, Ferreira AP, de Oliveira FF, Assumpcao LV, Metze K, Ward LS. Usefulness of HBME-1, cytokeratin 19 and galectin-3 immunostaining in the diagnosis of thyroid malignancy. Histopathology 2005 October;47(4):391-401.

(102) Sanabria A, Carvalho AL, Piana dA, V, Pablo RJ, Vartanian JG, Rinaldo A et al. Is galectin-3 a good method for the detection of malignancy in patients with thyroid nodules and a cytologic diagnosis of "follicular neoplasm"? A critical appraisal of the evidence. Head Neck 2007 November;29(11):1046-54.

(103) De Micco C, Savchenko V, Giorgi R, Sebag F, Henry JF. Utility of malignancy markers in fine-needle aspiration cytology of thyroid nodules: comparison of Hector Battifora mesothelial antigen-1, thyroid peroxidase and dipeptidyl aminopeptidase IV. Br J Cancer 2008 February 26;98(4):818-23.

(104) Griffith OL, Chiu CG, Gown AM, Jones SJ, Wiseman SM. Biomarker panel diagnosis of thyroid cancer: a critical review. Expert Rev Anticancer Ther 2008 September;8(9):1399-413. 
(105) Shields JM, Pruitt K, McFall A, Shaub A, Der CJ. Understanding Ras: 'it ain't over 'til it's over'. Trends Cell Biol 2000 April;10(4):147-54.

(106) Xing M, Westra WH, Tufano RP, Cohen Y, Rosenbaum E, Rhoden KJ et al. BRAF mutation predicts a poorer clinical prognosis for papillary thyroid cancer. J Clin Endocrinol Metab 2005 December;90(12):6373-9.

(107) Fugazzola L, Puxeddu E, Avenia N, Romei C, Cirello V, Cavaliere A et al. Correlation between BRAFV600E mutation and clinico-pathologic parameters in papillary thyroid carcinoma: data from a multicentric Italian study and review of the literature. Endocr Relat Cancer 2006 June;13(2):455-64.

(108) Nikiforova MN, Kimura ET, Gandhi M, Biddinger PW, Knauf JA, Basolo F et al. BRAF mutations in thyroid tumors are restricted to papillary carcinomas and anaplastic or poorly differentiated carcinomas arising from papillary carcinomas. J Clin Endocrinol Metab 2003 November;88(11):5399-404.

(109) Xing M. BRAF mutation in papillary thyroid cancer: pathogenic role, molecular bases, and clinical implications. Endocr Rev 2007 December;28(7):742-62.

(110) Xing M. BRAF mutation in papillary thyroid microcarcinoma: the promise of better risk management. Ann Surg Oncol 2009 April;16(4):801-3.

(111) Knauf JA, Fagin JA. Role of MAPK pathway oncoproteins in thyroid cancer pathogenesis and as drug targets. Curr Opin Cell Biol 2009 April;21(2):296-303.

(112) Hay ID, Bergstralh EJ, Goellner JR, Ebersold JR, Grant CS. Predicting outcome in papillary thyroid carcinoma: development of a reliable prognostic scoring system in a cohort of 1779 patients surgically treated at one institution during 1940 through 1989. Surgery 1993 December;114(6):1050-7.

(113) Mazzaferri EL, Jhiang SM. Long-term impact of initial surgical and medical therapy on papillary and follicular thyroid cancer. Am J Med 1994 November;97(5):418-28.

(114) Gilliland FD, Hunt WC, Morris DM, Key CR. Prognostic factors for thyroid carcinoma. A populationbased study of 15,698 cases from the Surveillance, Epidemiology and End Results (SEER) program 1973-1991. Cancer 1997 February 1;79(3):564-73.

(115) Lee JH, Lee ES, Kim YS. Clinicopathologic significance of BRAF V600E mutation in papillary carcinomas of the thyroid: a meta-analysis. Cancer 2007 July 1;110(1):38-46.

(116) Cheng S, Serra S, Mercado M, Ezzat S, Asa SL. A high-throughput proteomic approach provides distinct signatures for thyroid cancer behavior. Clin Cancer Res 2011 April 15;17(8):2385-94.

(117) Eberhardt NL, Grebe SK, McIver B, Reddi HV. The role of the PAX8/PPARgamma fusion oncogene in the pathogenesis of follicular thyroid cancer. Mol Cell Endocrinol 2010 May 28;321(1):50-6.

(118) Castro P, Roque L, Magalhaes J, Sobrinho-Simoes M. A subset of the follicular variant of papillary thyroid carcinoma harbors the PAX8-PPARgamma translocation. Int J Surg Pathol 2005 July;13(3):235-8.

(119) Castro P, Rebocho AP, Soares RJ, Magalhaes J, Roque L, Trovisco V et al. PAX8-PPARgamma rearrangement is frequently detected in the follicular variant of papillary thyroid carcinoma. J Clin Endocrinol Metab 2006 January;91(1):213-20.

(120) French CA, Alexander EK, Cibas ES, Nose V, Laguette J, Faquin W et al. Genetic and biological subgroups of low-stage follicular thyroid cancer. Am J Pathol 2003 April;162(4):1053-60.

(121) Nikiforova MN, Lynch RA, Biddinger PW, Alexander EK, Dorn GW, Tallini G et al. RAS point mutations and PAX8-PPAR gamma rearrangement in thyroid tumors: evidence for distinct molecular pathways in thyroid follicular carcinoma. J Clin Endocrinol Metab 2003 May;88(5):2318-26.

(122) Kroll TG, Sarraf P, Pecciarini L, Chen CJ, Mueller E, Spiegelman BM et al. PAX8-PPARgamma1 fusion oncogene in human thyroid carcinoma [corrected]. Science 2000 August 25;289(5483):1357-60.

(123) Reddi HV, Madde P, Milosevic D, Hackbarth JS, geciras-Schimnich A, McIver B et al. The Putative PAX8/PPARgamma Fusion Oncoprotein Exhibits Partial Tumor Suppressor Activity through UpRegulation of Micro-RNA-122 and Dominant-Negative PPARgamma Activity. Genes Cancer 2011 January;2(1):46-55.

(124) Nikiforova MN, Biddinger PW, Caudill CM, Kroll TG, Nikiforov YE. PAX8-PPARgamma rearrangement in thyroid tumors: RT-PCR and immunohistochemical analyses. Am J Surg Pathol 2002 August;26(8):1016-23.

(125) Zhu Z, Gandhi M, Nikiforova MN, Fischer AH, Nikiforov YE. Molecular profile and clinicalpathologic features of the follicular variant of papillary thyroid carcinoma. An unusually high prevalence of ras mutations. Am J Clin Pathol 2003 July;120(1):71-7.

(126) Adeniran AJ, Zhu Z, Gandhi M, Steward DL, Fidler JP, Giordano TJ et al. Correlation between genetic alterations and microscopic features, clinical manifestations, and prognostic characteristics of thyroid papillary carcinomas. Am J Surg Pathol 2006 February;30(2):216-22.

(127) Cooper DS, Doherty GM, Haugen BR, Kloos RT, Lee SL, Mandel SJ et al. Management guidelines for patients with thyroid nodules and differentiated thyroid cancer. Thyroid 2006 February;16(2):109-42. 
(128) White ML, Gauger PG, Doherty GM. Central lymph node dissection in differentiated thyroid cancer. World J Surg 2007 May;31(5):895-904.

(129) Musacchio MJ, Kim AW, Vijungco JD, Prinz RA. Greater local recurrence occurs with "berry picking" than neck dissection in thyroid cancer. Am Surg 2003 March;69(3):191-6.

(130) Sipos JA, Mazzaferri EL. The therapeutic management of differentiated thyroid cancer. Expert Opin Pharmacother 2008 October;9(15):2627-37.

(131) Bardet S, Malville E, Rame JP, Babin E, Samama G, De RD et al. Macroscopic lymph-node involvement and neck dissection predict lymph-node recurrence in papillary thyroid carcinoma. Eur $\mathbf{J}$ Endocrinol 2008 April;158(4):551-60.

(132) Mazzaferri EL, Doherty GM, Steward DL. The pros and cons of prophylactic central compartment lymph node dissection for papillary thyroid carcinoma. Thyroid 2009 July;19(7):683-9.

(133) Mazzaferri EL. What is the optimal initial treatment of low-risk papillary thyroid cancer (and why is it controversial)? Oncology (Williston Park) 2009 June;23(7):579-88.

(134) Rosenbaum MA, McHenry CR. Central neck dissection for papillary thyroid cancer. Arch Otolaryngol Head Neck Surg 2009 November;135(11):1092-7.

(135) Bozec A, Dassonville O, Chamorey E, Poissonnet G, Sudaka A, Peyrottes I et al. Clinical impact of cervical lymph node involvement and central neck dissection in patients with papillary thyroid carcinoma: a retrospective analysis of 368 cases. Eur Arch Otorhinolaryngol 2011 August;268(8):120512.

(136) So YK, Seo MY, Son YI. Prophylactic central lymph node dissection for clinically node-negative papillary thyroid microcarcinoma: Influence on serum thyroglobulin level, recurrence rate, and postoperative complications. Surgery 2011 April 15.

(137) Wingert DJ, Friesen SR, Iliopoulos JI, Pierce GE, Thomas JH, Hermreck AS. Post-thyroidectomy hypocalcemia. Incidence and risk factors. Am J Surg 1986 December;152(6):606-10.

(138) Chao TC, Jeng LB, Lin JD, Chen MF. Reoperative thyroid surgery. World J Surg 1997 July;21(6):6447.

(139) Mirghani H, Francois A, Landry G, Hans S, Menard M, Brasnu D. [Repeat of lymphatic dissection for thyroid cancers]. Ann Otolaryngol Chir Cervicofac 2009 April;126(2):37-42.

(140) Lewis BD, Hay ID, Charboneau JW, McIver B, Reading CC, Goellner JR. Percutaneous ethanol injection for treatment of cervical lymph node metastases in patients with papillary thyroid carcinoma. AJR Am J Roentgenol 2002 March;178(3):699-704.

(141) Mazzaferri EL, Kloos RT. Clinical review 128: Current approaches to primary therapy for papillary and follicular thyroid cancer. J Clin Endocrinol Metab 2001 April;86(4):1447-63.

(142) Haymart MR, Banerjee M, Stewart AK, Koenig RJ, Birkmeyer JD, Griggs JJ. Use of radioactive iodine for thyroid cancer. JAMA 2011 August 17;306(7):721-8.

(143) Almeida JP, Sanabria AE, Lima EN, Kowalski LP. Late side effects of radioactive iodine on salivary gland function in patients with thyroid cancer. Head Neck 2011 May;33(5):686-90.

(144) Van Nostrand D. The benefits and risks of I-131 therapy in patients with well-differentiated thyroid cancer. Thyroid 2009 December;19(12):1381-91.

(145) Rubino C, De VF, Dottorini ME, Hall P, Schvartz C, Couette JE et al. Second primary malignancies in thyroid cancer patients. Br J Cancer 2003 November 3;89(9):1638-44.

(146) Iyer NG, Morris LG, Tuttle RM, Shaha AR, Ganly I. Rising incidence of second cancers in patients with low-risk (T1N0) thyroid cancer who receive radioactive iodine therapy. Cancer 2011 October 1;117(19):4439-46.

(147) Sawka AM, Thephamongkhol K, Brouwers M, Thabane L, Browman G, Gerstein HC. Clinical review 170: A systematic review and metaanalysis of the effectiveness of radioactive iodine remnant ablation for well-differentiated thyroid cancer. J Clin Endocrinol Metab 2004 August;89(8):3668-76.

(148) Jonklaas J, Sarlis NJ, Litofsky D, Ain KB, Bigos ST, Brierley JD et al. Outcomes of patients with differentiated thyroid carcinoma following initial therapy. Thyroid 2006 December;16(12):1229-42.

(149) Takami H, Ito Y, Okamoto T, Yoshida A. Therapeutic strategy for differentiated thyroid carcinoma in Japan based on a newly established guideline managed by Japanese Society of Thyroid Surgeons and Japanese Association of Endocrine Surgeons. World J Surg 2011 January;35(1):111-21.

(150) Schlumberger M, Berg G, Cohen O, Duntas L, Jamar F, Jarzab B et al. Follow-up of low-risk patients with differentiated thyroid carcinoma: a European perspective. Eur J Endocrinol 2004 February;150(2):105-12.

(151) Lee N, Tuttle M. The role of external beam radiotherapy in the treatment of papillary thyroid cancer. Endocr Relat Cancer 2006 December;13(4):971-7.

(152) Kim TH, Chung KW, Lee YJ, Park CS, Lee EK, Kim TS et al. The effect of external beam radiotherapy volume on locoregional control in patients with locoregionally advanced or recurrent nonanaplastic thyroid cancer. Radiat Oncol 2010;5:69. 
(153) Kim DW, Jo YS, Jung HS, Chung HK, Song JH, Park KC et al. An orally administered multitarget tyrosine kinase inhibitor, SU11248, is a novel potent inhibitor of thyroid oncogenic RET/papillary thyroid cancer kinases. J Clin Endocrinol Metab 2006 October;91(10):4070-6.

(154) Salvatore G, De F, V, Salerno P, Nappi TC, Pepe S, Troncone G et al. BRAF is a therapeutic target in aggressive thyroid carcinoma. Clin Cancer Res 2006 March 1;12(5):1623-9.

(155) Gupta-Abramson V, Troxel AB, Nellore A, Puttaswamy K, Redlinger M, Ransone K et al. Phase II trial of sorafenib in advanced thyroid cancer. J Clin Oncol 2008 October 10;26(29):4714-9.

(156) Kloos RT, Ringel MD, Knopp MV, Hall NC, King M, Stevens R et al. Phase II trial of sorafenib in metastatic thyroid cancer. J Clin Oncol 2009 April 1;27(10):1675-84.

(157) Wedge SR, Ogilvie DJ, Dukes M, Kendrew J, Chester R, Jackson JA et al. ZD6474 inhibits vascular endothelial growth factor signaling, angiogenesis, and tumor growth following oral administration. Cancer Res 2002 August 15;62(16):4645-55.

(158) Herbst RS, Heymach JV, O'Reilly MS, Onn A, Ryan AJ. Vandetanib (ZD6474): an orally available receptor tyrosine kinase inhibitor that selectively targets pathways critical for tumor growth and angiogenesis. Expert Opin Investig Drugs 2007 February;16(2):239-49.

(159) Wells SA, Jr., Robinson BG, Gagel RF, Dralle H, Fagin JA, Santoro M et al. Vandetanib in Patients With Locally Advanced or Metastatic Medullary Thyroid Cancer: A Randomized, Double-Blind Phase III Trial. J Clin Oncol 2011 October 24.

(160) Xing M, Clark D, Guan H, Ji M, Dackiw A, Carson KA et al. BRAF mutation testing of thyroid fineneedle aspiration biopsy specimens for preoperative risk stratification in papillary thyroid cancer. J Clin Oncol 2009 June 20;27(18):2977-82.

(161) Nikiforov YE, Steward DL, Robinson-Smith TM, Haugen BR, Klopper JP, Zhu Z et al. Molecular testing for mutations in improving the fine-needle aspiration diagnosis of thyroid nodules. J Clin Endocrinol Metab 2009 June;94(6):2092-8.

(162) Frasoldati A, Toschi E, Zini M, Flora M, Caroggio A, Dotti C et al. Role of thyroglobulin measurement in fine-needle aspiration biopsies of cervical lymph nodes in patients with differentiated thyroid cancer. Thyroid 1999 February;9(2):105-11.

(163) Boi F, Baghino G, Atzeni F, Lai ML, Faa G, Mariotti S. The diagnostic value for differentiated thyroid carcinoma metastases of thyroglobulin $(\mathrm{Tg})$ measurement in washout fluid from fine-needle aspiration biopsy of neck lymph nodes is maintained in the presence of circulating anti-Tg antibodies. J Clin Endocrinol Metab 2006 April;91(4):1364-9.

(164) Pacini F, Mariotti S, Formica N, Elisei R, Anelli S, Capotorti E et al. Thyroid autoantibodies in thyroid cancer: incidence and relationship with tumour outcome. Acta Endocrinol (Copenh) 1988 November;119(3):373-80.

(165) Spencer CA, Takeuchi M, Kazarosyan M, Wang CC, Guttler RB, Singer PA et al. Serum thyroglobulin autoantibodies: prevalence, influence on serum thyroglobulin measurement, and prognostic significance in patients with differentiated thyroid carcinoma. J Clin Endocrinol Metab 1998 April;83(4):1121-7.

(166) Paus E, Risberg T. Establishment and evaluation of a radioimmunoassay for neuron-specific enolase. A marker for small cell lung cancer. Tumour Biol 1989;10(1):23-30.

(167) Hagan AK, Zuchner T. Lanthanide-based time-resolved luminescence immunoassays. Anal Bioanal Chem 2011 July;400(9):2847-64.

(168) Cerutti JM, Delcelo R, Amadei MJ, Nakabashi C, Maciel RM, Peterson B et al. A preoperative diagnostic test that distinguishes benign from malignant thyroid carcinoma based on gene expression. $\mathrm{J}$ Clin Invest 2004 April;113(8):1234-42.

(169) Cerutti JM, Latini FR, Nakabashi C, Delcelo R, Andrade VP, Amadei MJ et al. Diagnosis of suspicious thyroid nodules using four protein biomarkers. Clin Cancer Res 2006 June 1;12(11 Pt 1):3311-8.

(170) Bertheau P, Cazals-Hatem D, Meignin V, de RA, Verola O, Lesourd A et al. Variability of immunohistochemical reactivity on stored paraffin slides. J Clin Pathol 1998 May;51(5):370-4.

(171) Ljung BM, Drejet A, Chiampi N. The factors important for successful fine-needle aspiration biopsies: a comparative study of breast diagnoses at two hospitals. Pathology (Phila) 1996;4(2):493-8.

(172) National Guidelines for Management of Well-Differentiated Thyroid Carcinomas. 2006. http://www.legeforeningen.no/asset/45263/1/45263_1.pdf

(173) Baloch ZW, Tam D, Langer J, Mandel S, Livolsi VA, Gupta PK. Ultrasound-guided fine-needle aspiration biopsy of the thyroid: role of on-site assessment and multiple cytologic preparations. Diagn Cytopathol 2000 December;23(6):425-9.

(174) Tollin SR, Mery GM, Jelveh N, Fallon EF, Mikhail M, Blumenfeld W et al. The use of fine-needle aspiration biopsy under ultrasound guidance to assess the risk of malignancy in patients with a multinodular goiter. Thyroid 2000 March;10(3):235-41.

(175) Rausch P, Nowels K, Jeffrey RB, Jr. Ultrasonographically guided thyroid biopsy: a review with emphasis on technique. J Ultrasound Med 2001 January;20(1):79-85. 
(176) Zajdela A, Zillhardt P, Voillemot N. Cytological diagnosis by fine needle sampling without aspiration. Cancer 1987 March 15;59(6):1201-5.

(177) Cappelli C, Pirola I, Castellano M, Gandossi E, De ME, Delbarba A et al. Fine needle cytology of complex thyroid nodules. Eur J Endocrinol 2007 October;157(4):529-32.

(178) Cappelli C, Pirola I, Gandossi E, De ME, Agosti B, Castellano M. Fine-needle aspiration cytology of thyroid nodule: does the needle matter? South Med J 2009 May;102(5):498-501.

(179) Baskin HJ. Detection of recurrent papillary thyroid carcinoma by thyroglobulin assessment in the needle washout after fine-needle aspiration of suspicious lymph nodes. Thyroid 2004 November;14(11):959-63.

(180) Uruno T, Miyauchi A, Shimizu K, Tomoda C, Takamura Y, Ito Y et al. Usefulness of thyroglobulin measurement in fine-needle aspiration biopsy specimens for diagnosing cervical lymph node metastasis in patients with papillary thyroid cancer. World J Surg 2005 April;29(4):483-5.

(181) Snozek CL, Chambers EP, Reading CC, Sebo TJ, Sistrunk JW, Singh RJ et al. Serum thyroglobulin, high-resolution ultrasound, and lymph node thyroglobulin in diagnosis of differentiated thyroid carcinoma nodal metastases. J Clin Endocrinol Metab 2007 November;92(11):4278-81.

(182) Cunha N, Rodrigues F, Curado F, Ilheu O, Cruz C, Naidenov P et al. Thyroglobulin detection in fineneedle aspirates of cervical lymph nodes: a technique for the diagnosis of metastatic differentiated thyroid cancer. Eur J Endocrinol 2007 July;157(1):101-7.

(183) Baloch ZW, Barroeta JE, Walsh J, Gupta PK, Livolsi VA, Langer JE et al. Utility of Thyroglobulin measurement in fine-needle aspiration biopsy specimens of lymph nodes in the diagnosis of recurrent thyroid carcinoma. Cytojournal 2008;5:1.

(184) Borel AL, Boizel R, Faure P, Barbe G, Boutonnat J, Sturm N et al. Significance of low levels of thyroglobulin in fine needle aspirates from cervical lymph nodes of patients with a history of differentiated thyroid cancer. Eur J Endocrinol 2008 May;158(5):691-8.

(185) Jeon SJ, Kim E, Park JS, Son KR, Baek JH, Kim YS et al. Diagnostic benefit of thyroglobulin measurement in fine-needle aspiration for diagnosing metastatic cervical lymph nodes from papillary thyroid cancer: correlations with US features. Korean J Radiol 2009 March;10(2):106-11.

(186) Salmaslioglu A, Erbil Y, Citlak G, Ersoz F, Sari S, Olmez A et al. Diagnostic value of thyroglobulin measurement in fine-needle aspiration biopsy for detecting metastatic lymph nodes in patients with papillary thyroid carcinoma. Langenbecks Arch Surg 2011 January;396(1):77-81.

(187) Solbiati L, Giangrande A, De PL, Bellotti E, Cantu P, Ravetto C. Percutaneous ethanol injection of parathyroid tumors under US guidance: treatment for secondary hyperparathyroidism. Radiology 1985 June;155(3):607-10.

(188) Karstrup S, Transbol I, Holm HH, Glenthoj A, Hegedus L. Ultrasound-guided chemical parathyroidectomy in patients with primary hyperparathyroidism: a prospective study. Br J Radiol 1989 December;62(744):1037-42.

(189) Livraghi T, Paracchi A, Ferrari C, Reschini E, Macchi RM, Bonifacino A. Treatment of autonomous thyroid nodules with percutaneous ethanol injection: 4-year experience. Radiology 1994 February;190(2):529-33.

(190) Rossi R, Savastano S, Tommaselli AP, Valentino R, Iaccarino V, Tauchmanova L et al. Percutaneous computed tomography-guided ethanol injection in aldosterone-producing adrenocortical adenoma. Eur $\mathrm{J}$ Endocrinol 1995 March;132(3):302-5.

(191) Livraghi T. Treatment of hepatocellular carcinoma by interventional methods. Eur Radiol 2001;11(11):2207-19.

(192) Giovannini M. Percutaneous alcohol ablation for liver metastasis. Semin Oncol 2002 April;29(2):192-5.

(193) Martino E, Murtas ML, Loviselli A, Piga M, Petrini L, Miccoli P et al. Percutaneous intranodular ethanol injection for treatment of autonomously functioning thyroid nodules. Surgery 1992 December;112(6):1161-4.

(194) Cerbone G, Spiezia S, Colao A, Marzullo P, Assanti AP, Lucci R et al. Percutaneous ethanol injection under Power Doppler ultrasound assistance in the treatment of autonomously functioning thyroid nodules. J Endocrinol Invest 1999 November;22(10):752-9.

(195) Spiezia S, Cerbone G, Assanti AP, Colao A, Siciliani M, Lombardi G. Power Doppler ultrasonographic assistance in percutaneous ethanol injection of autonomously functioning thyroid nodules. J Ultrasound Med 2000 January;19(1):39-46.

(196) Goletti O, Monzani F, Lenziardi M, Lippolis PV, De NF, Caraccio N et al. Cold thyroid nodules: a new application of percutaneous ethanol injection treatment. J Clin Ultrasound 1994 March;22(3):175-8.

(197) Zingrillo M, Collura D, Ghiggi MR, Nirchio V, Trischitta V. Treatment of large cold benign thyroid nodules not eligible for surgery with percutaneous ethanol injection. J Clin Endocrinol Metab 1998 November;83(11):3905-7. 
(198) Monzani F, Lippi F, Goletti O, Del GP, Caraccio N, Lippolis PV et al. Percutaneous aspiration and ethanol sclerotherapy for thyroid cysts. J Clin Endocrinol Metab 1994 March;78(3):800-2.

(199) Zingrillo M, Torlontano M, Chiarella R, Ghiggi MR, Nirchio V, Bisceglia M et al. Percutaneous ethanol injection may be a definitive treatment for symptomatic thyroid cystic nodules not treatable by surgery: five-year follow-up study. Thyroid 1999 August;9(8):763-7.

(200) Cho YS, Lee HK, Ahn IM, Lim SM, Kim DH, Choi CG et al. Sonographically guided ethanol sclerotherapy for benign thyroid cysts: results in 22 patients. AJR Am J Roentgenol 2000 January; 174(1):213-6.

(201) Hay ID, Charboneau JW. The coming of age of ultrasound-guided percutaneous ethanol ablation of selected neck nodal metastases in well-differentiated thyroid carcinoma. J Clin Endocrinol Metab 2011 September;96(9):2717-20.

(202) Monchik JM, DeLellis RA. Re-operative neck surgery for well-differentiated thyroid cancer of follicular origin. J Surg Oncol 2006 December 15;94(8):714-8.

(203) Levin KE, Clark AH, Duh QY, Demeure M, Siperstein AE, Clark OH. Reoperative thyroid surgery. Surgery 1992 June;111(6):604-9.

(204) Shaha AR, Jaffe BM. Parathyroid preservation during thyroid surgery. Am J Otolaryngol 1998 March;19(2):113-7.

(205) Sawka AM, Brierley JD, Tsang RW, Thabane L, Rotstein L, Gafni A et al. An updated systematic review and commentary examining the effectiveness of radioactive iodine remnant ablation in welldifferentiated thyroid cancer. Endocrinol Metab Clin North Am 2008 June;37(2):457-80, x.

(206) van Roosmalen J., van HB, Suurmeijer A, Groen H, Ruitenbeek T, Links TP et al. Diagnostic value and cost considerations of routine fine-needle aspirations in the follow-up of thyroid nodules with benign readings. Thyroid 2010 December;20(12):1359-65.

(207) Pfeffer C, Olsen BR. Editorial: Journal of negative results in biomedicine. J Negat Results Biomed 2002;1:2.

(208) Hamady ZZ, Mather N, Lansdown MR, Davidson L, Maclennan KA. Surgical pathological second opinion in thyroid malignancy: impact on patients' management and prognosis. Eur J Surg Oncol 2005 February;31(1):74-7.

(209) Giovanella L, Ceriani L, Suriano S, Crippa S. Thyroglobulin measurement on fine-needle washout fluids: Influence of sample collection methods. Diagn Cytopathol 2009 January;37(1):42-4.

(210) Personalized Health Care Expert Panel Meeting: Summary Report. 2007.

Ref Type: Internet Communication

(211) American Thyroid Association (ATA). Thyroid Disorders in the Era of Personalized Medicine. Thyroid 2010;20(7).

(212) The Manual of Cancer Services Standards UK. 2004.

http://www.dh.gov.uk/cancer

(213) National Institute of Clinical Excellence. Improving Outcomes Guidance (IOG). Head and Neck Cancer. The Manual. 2004. http://www.nice.org.uk/

(214) Baudin E, Schlumberger M. New therapeutic approaches for metastatic thyroid carcinoma. Lancet Oncol 2007 February;8(2):148-56.

(215) Ohori NP, Nikiforova MN, Schoedel KE, Lebeau SO, Hodak SP, Seethala RR et al. Contribution of molecular testing to thyroid fine-needle aspiration cytology of "follicular lesion of undetermined significance/atypia of undetermined significance". Cancer Cytopathol 2010 February 25;118(1):17-23.

(216) Nikiforov YE, Ohori NP, Hodak SP, Carty SE, Lebeau SO, Ferris RL et al. Impact of Mutational Testing on the Diagnosis and Management of Patients with Cytologically Indeterminate Thyroid Nodules: A Prospective Analysis of 1056 FNA Samples. J Clin Endocrinol Metab 2011 August 31.

(217) Hayashida N, Namba H, Kumagai A, Hayashi T, Ohtsuru A, Ito M et al. A rapid and simple detection method for the BRAF(T1796A) mutation in fine-needle aspirated thyroid carcinoma cells. Thyroid 2004 November;14(11):910-5.

(218) Jin L, Sebo TJ, Nakamura N, Qian X, Oliveira A, Majerus JA et al. BRAF mutation analysis in fine needle aspiration (FNA) cytology of the thyroid. Diagn Mol Pathol 2006 September;15(3):136-43.

(219) Sapio MR, Posca D, Troncone G, Pettinato G, Palombini L, Rossi G et al. Detection of BRAF mutation in thyroid papillary carcinomas by mutant allele-specific PCR amplification (MASA). Eur J Endocrinol 2006 February; 154(2):341-8.

(220) Rowe LR, Bentz BG, Bentz JS. Utility of BRAF V600E mutation detection in cytologically indeterminate thyroid nodules. Cytojournal 2006;3:10. 


\section{Errata}

1. There is a typing error in Paper I in Table III. The headings of the columns indicating which hospital the specimens originate from are wrong. The heading " $\mathrm{B}$ " should be replaced by "A", and the heading " $H$ " should be replaced by "B".

2. There is a typing error in Paper I in Abstract. The number of specimens diagnosed from the two community Hospitals A and B were 424, not 425 as mentioned in the abstract. Through the rest of the paper, the correct number of 424 is used.

3. In Paper I, the calculated specificity for NRH and community Hopsitals A and B is incorrect and should be $100 \%$ for community Hospitals A and B and $99.6 \%$ for NRH.

4. There is a typing error in Paper III in paragraph "Patients and Methods" repeating the sentence "Hybridoma were selected in presence of autoantibodies in order to obtain minimal interference from human anti-Tg antibodies in serum when used in the assay for $\mathrm{Tg} "$

5. Reference number 4 and 20 in Paper I have through an oversight been found to be the same reference. For this reference (by Ravetto et al) the correct page numbers in Cancer 2000;90 are 357-363 as written in reference number 4, not page numbers 325329 as written in reference number 20.

6. Through an oversight the URL-link for reference number 210 in the Thesis has been found missing. The correct URL-link is: http://aspe.hhs.gov/health/reports/07/PHC/execsum.htm 




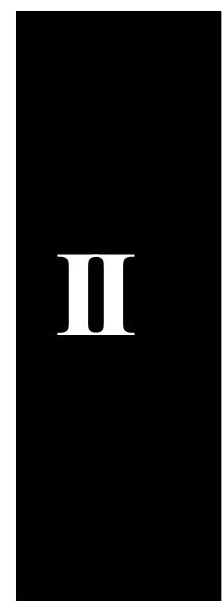





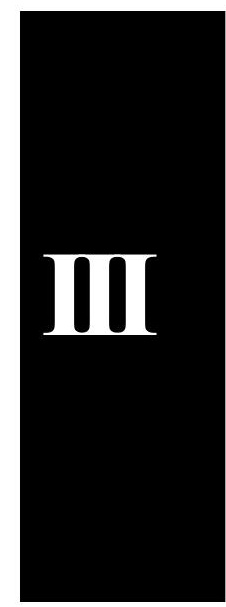





\title{
Efficacy of Ultrasound-Guided Percutaneous Ethanol Injection Treatment in Patients with a Limited Number of Metastatic Cervical Lymph Nodes from Papillary Thyroid Carcinoma
}

\author{
Arne Heilo, Eva Sigstad, Kristin Holgersen Fagerlid, Olav Inge Håskjold, \\ Krystyna Kotanska Grøholt, Aasmund Berner, Trine Bjøro, and Lars H. Jørgensen \\ Departments of Radiology and Nuclear Medicine (A.H., K.H.F., O.I.H.), Pathology (E.S., K.K.G., A.B.), and \\ Medical Biochemistry (T.B.), Division of Diagnostics and Intervention; and Department of Thoracic \\ Surgery, Division of Cardiovascular and Pulmonary Diseases (L.H.J.), Oslo University Hospital HF, 0424 \\ Oslo, Norway; and Faculty of Medicine (A.B., T.B.), University of Oslo, 0316 Oslo, Norway
}

\begin{abstract}
Context: Repeated neck explorations can be a difficult task in patients with recurrent metastatic cervical lymph nodes from papillary thyroid carcinoma (PTC).

Objective: The aim of this retrospective study has been to assess the efficacy of ultrasound (US)-guided percutaneous ethanol injection (PEI) as treatment of metastatic cervical lymph nodes from PTC.

Materials and Methods: Sixty-nine patients who previously had undergone thyroidectomy for PTC were selected for inclusion. However, three patients were later excluded due to lack of follow-up. Lymph node status was determined by US-guided fine-needle aspiration biopsy and/or by raised levels of thyroglobulin in washouts from the cytological needle. Guided by US, $0.1-1.0 \mathrm{ml}$ of $99.5 \%$ ethanol was injected into the metastatic lymph nodes.

Results: Three patients (eight metastatic lymph nodes in total) were reassigned to surgery due to progression (multiple new metastases), leaving 63 patients and 109 neck lymph nodes to be included. Mean observation time was 38.4 months (range, 3-72). A total of 101 of the 109 (93\%) metastatic lymph nodes responded to PEI treatment, 92 (84\%) completely and nine incompletely. Two did not respond, and four progressed. Two lymph nodes previously considered successfully treated showed evidence of malignancy during follow-up. No significant side effects were reported.
\end{abstract}

Conclusion: US-guided PEI treatment of metastatic lymph nodes seems to be an excellent alternative to surgery in patients with a limited number of neck metastases from PTC. This procedure should replace "berry picking" surgery. (J Clin Endocrinol Metab 96: 2750-2755, 2011)

$P$ pillary thyroid carcinoma (PTC) represents about $80 \%$ of all thyroid cancers (1). Most PTC are indolent, with a 10 -yr cause-specific survival rate of $93 \%$ regardless of stage $(2,3)$. Total thyroidectomy is the most common treatment. In patients with low-risk PTC, neck recurrence occurs in up to $20 \%(4,5)$. The benefits of concomitant lymph node resection in the central neck compartment are questionable

ISSN Print 0021-972X ISSN Online 1945-7197

Printed in U.S.A.

Copyright @ 2011 by The Endocrine Society

doi: 10.1210/jc.2010-2952 Received December 17, 2010. Accepted June 10, 2011 First Published Online June 29, 2011

For article see page 2717 and a matter of constant debate (6). The American Thyroid Association recently (2009) revised their guidelines for neck surgery on PTC patients, concluding that "prophylactic central-compartment neck dissection (ipsilateral or bilateral) is recommended" (7).

Depending on size and multiplicity of primary thyroid tumor, occurrence of metastatic lymph nodes in the neck, or

Abbreviations: FNAB, Fine-needle aspiration biopsy; FNAB-Tg, measurement of Tg in washout needle aspiration biopsy; TNM, tumor node metastasis; PEI, percutaneous ethanol injection; PTC, papillary thyroid carcinoma; S-Tg, serum Tg; S-TgAb, S-Tg antibodies; Tg, thyroglobulin; US, ultrasound. 
distant metastases, the primary operation is followed by ablation therapy with ${ }^{131} \mathrm{I}$. Unfortunately, radioiodine treatment is often ineffective in eradicating lymph node metastases (8). Due to the mild clinical course, many patients undergo repeated neck explorations when additional metastases are revealed. Such explorations are usually challenging due to scar formation subsequent to previous surgery, yielding a higher risk of complications (9).

Ultrasound (US) and serum thyroglobulin (S-Tg) measurements are the most important examinations during follow-up after primary treatment for PTC. US may detect metastatic lymph nodes as small as $2 \mathrm{~mm}$. Fine-needle aspiration biopsies (FNAB) and thyroglobulin (Tg) measurements in needle washouts (FNAB-Tg) have high sensitivity and specificity in verifying lymph node metastases (10).

Different authors have reported good results using percutaneous ethanol injection (PEI) in the treatment of patients with benign thyroid cysts $(11,12)$ as well as hyperfunctioning thyroid adenomas (13) and benign parathyroid adenomas (14). In 2002, Lewis et al. (15) reported promising results using US-guided PEI treatment of 14 patients with cervical lymph node metastases. This inspired our multidisciplinary thyroid team at the Norwegian Radium Hospital (NRH), and we consequently introduced the PEI method in 2004. Since then, three other studies comprising six, 16, and 27 patients, respectively, have reported promising results $(16-18)$.

\section{Patients and Methods}

\section{Patients}

From June 2004 through December 2009, 69 patients (27 men and 42 women) with PTC and cervical metastatic lymph nodes were retrospectively included in this study. All patients had undergone total or subtotal thyroidectomy in one or two steps, followed by radioiodine ablation. Three of the 69 patients were excluded due to lack of follow-up. Due to progression (appearance of new metastatic neck lymph nodes), three patients (eight metastatic lymph nodes in total) were reassigned for surgery. The remaining 63 patients ( 109 neck lymph nodes in total) were treated with PEI as monotherapy. The median age at primary surgery was $43 \mathrm{yr}$ (range, 20-76), and median age at PEI was 52 yr (range, 21-85).

Clinical information and tumor node metastasis (TNM) category at the time of primary diagnosis were retrospectively registered as shown in Table 1. All 63 patients had localized disease and had no distant metastasis (M0 category). Fifty-seven patients had undergone central neck lymph node dissection. Many patients had also undergone lateral neck node dissection. However, some had received surgical treatment in other hospitals, and detailed information about surgery, including extension of the central lymph node dissection at primary surgery, was not available.

Subsequent to surgery, the 63 patients were grouped as N1 (carcinoma has spread to nearby lymph nodes) ( $\mathrm{n}=46$ ), N0 (no
TABLE 1. Sixty-three patients with PTC categorized according to TNM classification

\begin{tabular}{ccrrr}
\hline $\begin{array}{c}\text { TNM classification } \\
\text { when first diagnosed }\end{array}$ & Total & N0 & N1 & NX \\
\hline T1 & 15 & 5 & 10 & 0 \\
T2 & 16 & 1 & 13 & 2 \\
T3 & 7 & 1 & 5 & 1 \\
T4 & 13 & 2 & 9 & 2 \\
Tx & 12 & 2 & 9 & 1 \\
Total & 63 & 11 & 46 & 6 \\
\hline
\end{tabular}

Information to classify the tumor was not available for 12 patients, and in six patients no information was available about the lymph nodes. All 63 patients had localized disease and had no distant metastases (MO category).

spread to nearly lymph nodes) $(\mathrm{n}=11)$, and NX (regional lymph nodes cannot be assessed) $(n=6)$ disease. All 63 patients had been treated with ${ }^{131}$ I once, twice, or several times (median, two times; range, 1-10 times) with median accumulated dose of ${ }^{131} \mathrm{I}$ of $284 \mathrm{mCi}(10.5 \mathrm{GBq})$ and range of $100-1406 \mathrm{mCi}$ (3.7-52 $\mathrm{GBq}$ ). Several patients (exact number impossible to determine due to lack of clinical information from local hospital) had undergone repeated neck dissections at NRH or a local hospital after initial treatment. One patient underwent seven surgical procedures in the course of $4 \mathrm{yr}$.

The interval from initial surgery to PEI treatment ranged from 6 to 384 months (median, 24 months).

None of the 63 patients included had more than three metastatic lymph nodes. The criteria for PEI treatment were fulfilled for all nodes treated.

\section{Methods}

The inclusion criteria for PEI treatment of PTC patients were as follows: 1) carcinoma cells identified in FNAB specimens and/or elevated level of FNAB-Tg; 2) no radiological findings suspicious of malignancy inferior to the neck; 3 ) record of previous treatment with ${ }^{131} \mathrm{I}$; and 4 ) detection of up to three metastatic lymph nodes in previously dissected neck compartments.

By US, all lymph nodes were visualized using a $12-5 \mathrm{MHz}$ broadband linear US transducer (HDI 5000 with Sono-CT and X-res; Philips-Advanced Technology Laboratories, Bothell, WA; or Logiq 9; GE Medical Systems, Milwaukee, WI).

The FNAB procedure, guided by US examination, was undertaken whenever a lymph node suspicious of metastatic disease was observed. The FNAB material was obtained using a 25 - or 27-gauge needle. The smears were immediately assessed on site by an experienced cytopathologist before PEI treatment. Metastasis was confirmed in cytological smears and/or by demonstration of raised levels of FNAB-Tg in needle washouts (10). The Bethesda classification system was used when reporting FNAB specimens (19). In cases with inconclusive or questionable FNAB, FNAB-Tg measurements ensured that no additional lymph node puncture was necessary. This multimodal approach including on-site US-guided FNAB examination and combined $\mathrm{Tg}$ measurements of needle washouts was a prerequisite for effective subsequent PEI treatment that was performed on most patients. All lymph nodes were grouped according to the American Joint Committee on Cancer, having levels from I-VII (20).

Due to promising results during the early phase of the study, patients with metastatic lymph nodes in nondissected compart- 
ments were eventually included. Up to five lymph nodes were injected if additional metastatic lymph nodes were detected during follow-up.

US examination was performed by three experienced radiologists (A.H., K.H.F., and O.I.H.). The US images allowed anteroposterior, transverse, and longitudinal measurements in most lymph nodes. Vascularity as displayed by color Doppler was always performed before treatment and whenever Doppler control was needed. The PEI procedure was always performed by the same radiologists. All patients were treated on an outpatient basis and without premedication. Only one patient needed local anesthetic. All patients were discharged immediately after treatment.

No coagulation tests were performed before the PEI treatment. S-Tg, S-Tg antibodies (S-TgAb), serum TSH, and serum free $\mathrm{T}_{4}$ were measured. S-Tg and FNAB-Tg were measured as previously described by a time-resolved immunofluorometric assay (10). A low, nonspecific binding was obtained, and the assay is characterized by an analytical detection limit of $0.1 \mu \mathrm{g} /$ liter and functional sensitivity of $0.2 \mu \mathrm{g} / \mathrm{liter}$. Tg antibodies were determined by Anti-TGn Kryptor from BRAHMS (Berlin, Germany) standardized with World Health Organization First International Reference Preparation 65/93. Tg antibodies value above $50 \mathrm{kU} /$ liter was considered positive, and 35-50 kU/liter was considered borderline positive. The detection limit was less than $10 \mathrm{kU} /$ liter.

The PEI technique used was essentially that described by Lewis et al. (15) using a 25 -gauge needle of $4 \mathrm{~cm}$ attached to a 1 -ml syringe filled with $99.5 \%$ ethanol. The US-guided puncture was performed by a freehand technique with the needle oriented at an angle parallel to the transducer scan plane. In total, 0.1 to $1 \mathrm{ml}$ ethanol was injected, starting with small amounts deposited in the peripheral part of the node and repeated several times during needle withdrawal. In larger nodes, the needle was repositioned into different parts of the node, and several injections were performed with the intention of achieving total ablation. All PEI-injected lymph nodes were monitored with color Doppler to display loss of vascularization, and if necessary further injections were performed in vascularized areas. The volume of injected ethanol and possible unwanted side effects were recorded.

Treatment was considered effective when reduction in volume and/or reduction of lymph node vascularization were demonstrated by US. Criteria for satisfactory PEI treatment were: 1) complete lymph node disappearance; 2 ) reduction of the anteroposterior lymph node diameter to $4 \mathrm{~mm}$ or less without visible vascularization; 3 ) normalization of lymph node size and appearance, and in some nodes scar formation; and 4) no detectable malignant cells in the FNAB specimens and no detectable Tg in needle washouts.

The majority of the patients were reexamined every third or fourth month with US. Whenever additional lymph nodes suspicious of malignancy were detected, FNAB was performed, and the FNAB-Tg level was assessed. The malignant lymph nodes underwent PEI treatment until satisfactory results were achieved. If not, surgical extirpation was performed.

The study has been approved by the quality assurance committee of Oslo University Hospital.

\section{Results}

Three patients (eight metastatic neck lymph nodes in total) were reassigned for surgery due to progression (appearance of new metastatic lymph nodes). One of these three patients was reassigned for surgery due to appearance of metastatic lymph nodes in several additional levels. The next patient received surgery after being treated with PEI twice (after 8 months), and the last patient after four treatments (12 months).

In the remaining 63 patients, all 109 lymph nodes treated with PEI were diagnosed either on cytological smears or by FNAB-Tg measurements. Malignant lymph nodes were localized to levels VI and VII, containing 46 and three lymph nodes, respectively. Another 37 lymph nodes were detected in level IV, 11 in level III, four in level $\mathrm{Va}$, two in level Vb, three in level IIa, one in level IIb, and finally two had a parapharyngeal location. The initial cytology was positive in 91 lymph nodes. FNAB-Tg confirmed malignancy in the other 18, although smears from 12 of the 18 cases were suspicious of malignancy. Cytology was benign in two cases, and four did not yield diagnostic material. Four cases without elevated FNAB-Tg $(<0.1 \mu \mathrm{g} /$ liter $)$ demonstrated malignant cells in the cytological smears. This was probably due to insufficient amounts of material in the needle washout specimens.

The lymph node volume before treatment was in the range of $0.01-3.56 \mathrm{ml}$ (mean, 0.34). The injected volume of ethanol in each session varied from 0.1 to $1 \mathrm{ml}$ (mean, 0.4). Seventy-six metastases had pathological vascularity, 11 had normal vascularity, and 22 showed no vascularity. In addition, 29 had microcalcifications, and 21 were partially cystic at US examination before PEI treatment.

Size reduction of a PEI-treated lymph node was an excellent indicator of evaluating response to treatment. In general, hypervascular metastases turned almost avascular immediately after terminating the ethanol injection. Reduction or absence of vascularity was also observed in PEI-treated lymph nodes during follow-up. Independent of size, treatment was continued as long as vascularity could be demonstrated. However, a few lymph nodes did not shrink, and some even increased in size after the first PEI treatment.

Ninety-two of the 109 PEI-treated lymph nodes with follow-up were successfully treated. Seventy-two nodes disappeared, and 20 were successfully treated according to criteria 2-4 mentioned as satisfactory following PEI treatment (Fig. 1). As shown in Table 2, up to five ethanol injections were needed to eradicate the metastatic tumor tissue. Variation in metastatic neck lymph node size relative to number of injections is also presented (Table 2). 


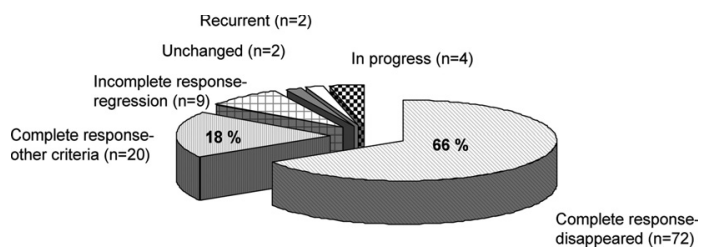

FIG. 1. The outcome of PEl treatment of neck lymph node metastases $(n=109)$.

We evacuated fluid from one cystic metastasis before PEI treatment.

At termination of the study, 17 neck lymph nodes still under treatment were followed by US. Nine of these demonstrated incomplete response after one or two injections (regression). Two were nonresponders (unchanged) after one injection, four progressed after two to six injections, and two had recurred (Fig. 1). Table 3 shows the minimum, maximum, and median diameter of all 109 lymph nodes before treatment and the posttreatment diameter of the 20 successfully treated lymph nodes that were still visible.

The US findings determined whether PEI treatment should be performed or not. Combined FNAB and FNAB-Tg examinations on 54 neck lymph nodes that had not disappeared were performed by US during follow-up. In 24 lymph nodes, FNAB examination detected malignant cells and/or detectable Tg in FNAB washouts that confirmed malignancy. Thus, further PEI was needed. Eighteen samples were negative by both methods, and the lymph nodes were classified as successfully treated. We did not achieve diagnostic FNAB from two lymph nodes; however, FNAB-Tg was negative and the lymph nodes were regarded as successfully treated. Samples from 10 lymph nodes did not yield diagnostic material by both methods due to scarring successive to PEI treatment.

Observation time after initial treatment varied from 3 to 72 months (mean, 38.4).

In agreement with others (15-18), we did not recognize any major complications related to PEI treatment, and less than $10 \%$ of patients experienced minor discomfort.

$\mathrm{S}-\mathrm{Tg}$ and $\mathrm{S}-\mathrm{TgAb}$ values were available from $62 \mathrm{pa}-$

TABLE 2. Volume of the metastases and number of ethanol injections performed in 92 successfully treated metastases

\begin{tabular}{cccc}
\hline $\begin{array}{c}\text { No. of } \\
\text { injections }\end{array}$ & $\begin{array}{c}\text { No. of } \\
\text { metastases }\end{array}$ & $\begin{array}{c}\text { Metastases volume } \\
\text { variation }(\mathrm{ml})\end{array}$ & $\begin{array}{c}\text { Mean met } \\
\text { volume }(\mathbf{m l})\end{array}$ \\
\hline 1 & 33 & $0.01-0.44$ & 0.14 \\
2 & 29 & $0.02-0.88$ & 0.28 \\
3 & 22 & $0.03-0.72$ & 0.29 \\
4 & 4 & $0.07-0.66$ & 0.38 \\
& 1 cystic & 3.56 & 0.77 \\
\hline
\end{tabular}

TABLE 3. Anteroposterior, transverse, and longitudinal diameters of the treated metastases

\begin{tabular}{|c|c|c|c|}
\hline & \multicolumn{3}{|c|}{ Diameter $(\mathrm{mm})$} \\
\hline & Minimum & Maximum & Median \\
\hline \multicolumn{4}{|c|}{$\begin{array}{l}\text { Pretreatment size of the } \\
72 \text { metastases that } \\
\text { disappeared after } \\
\text { treatment }\end{array}$} \\
\hline Anteroposterior & 1.8 & 10 & 3.4 \\
\hline Transverse & 2.3 & 16 & 7.0 \\
\hline Longitudinal & 3 & 27 & 9.2 \\
\hline \multicolumn{4}{|c|}{$\begin{array}{l}\text { Pretreatment size of the } \\
20 \text { successfully } \\
\text { treated metastases }\end{array}$} \\
\hline Anteroposterior & 2.8 & 16 & 5.0 \\
\hline Transverse & 3.7 & 21 & 10.0 \\
\hline Longitudinal & 4.3 & 27 & 10.5 \\
\hline \multicolumn{4}{|c|}{$\begin{array}{l}\text { Posttreatment size of } \\
\text { the } 20 \text { successfully } \\
\text { treated metastases }\end{array}$} \\
\hline Anteroposterior & 1.6 & 3.8 & 2.0 \\
\hline Transverse & 2.5 & 7 & 4.0 \\
\hline Longitudinal & 3 & 17 & 4.9 \\
\hline \multicolumn{4}{|c|}{$\begin{array}{l}\text { Pretreatment size of the } \\
17 \text { metastases still } \\
\text { under treatment }\end{array}$} \\
\hline Anteroposterior & 2.2 & 10 & 5.4 \\
\hline Transverse & 2.4 & 14 & 7.2 \\
\hline Longitudinal & 3.5 & 17 & 8.0 \\
\hline
\end{tabular}

tients before PEI and 60 at the end of follow-up. Before PEI, 53 of the 62 patients had S-TgAb levels less than 10 $\mathrm{kU} /$ liter, whereas nine patients had positive S-TgAb measurements (range, 60-1500 kU/liter). In three of the nine patients with detectable S-TgAb before PEI, S-TgAb was undetectable $(<10 \mathrm{kU} /$ liter $)$ at follow-up, indicating successful treatment. Of the 51 patients without S-TgAb, both initially and at the end point, 13 had undetectable S-Tg $(<0.2 \mu \mathrm{g} /$ liter $)$ before PEI despite metastatic disease. Median S-Tg was $0.7 \mu \mathrm{g} /$ liter (range, $<0.2-26 \mu \mathrm{g} /$ liter; $\mathrm{n}=51$ ) before PEI and less than $0.2 \mu \mathrm{g} /$ liter (range, $<0.2-31 \mu \mathrm{g} /$ liter; $\mathrm{n}=51$ ) after PEI. We did not detect $\mathrm{S}-\operatorname{Tg}(<0.2 \mu \mathrm{g} /$ liter $)$ or S-TgAb $(<10 \mathrm{kU} /$ liter $)$ in $43 \mathrm{pa}-$ tients after PEI treatment. Of the 38 patients with elevated S-Tg levels before PEI, 30 patients had undetectable S-Tg values $(<0.2 \mu \mathrm{g} /$ liter $)$ after treatment (Fig. 2). In eight patients, S-Tg levels were detectable $(0.37-31 \mu \mathrm{g} / \mathrm{liter})$, and in two patients, $\mathrm{Tg}$ levels were even increased at the end of follow-up, perhaps indicating as yet unidentified metastatic disease in the neck or elsewhere.

\section{Discussion}

A multidisciplinary approach to the diagnosis and treatment of thyroid tumors is crucial. For several years, there 


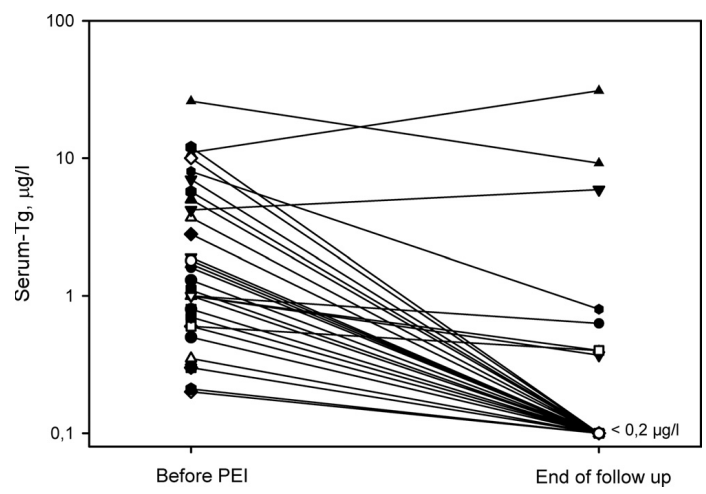

FIG. 2. S-Tg values in patients with undetectable S-TgAb before PEI treatment and at end of follow-up and S-Tg of at least $0.2 \mu \mathrm{g} / \mathrm{liter}$ ( $n=38$ ) before PEl. Functional sensitivity of the Tg assay is $0.2 \mu \mathrm{g} /$ liter, and S-Tg values less than $0.2 \mu \mathrm{g} /$ liter are presented as $0.1 \mu \mathrm{g} /$ liter. Thirteen patients with S-Tg less than $0.2 \mu \mathrm{g} /$ liter both before and at end of follow-up are not included in the figure.

have existed international and national guidelines (http:// www.legeforeningen.no/asset/45263/1/45263_1.pdf) regarding the management of patients with thyroid nodules and differentiated thyroid cancer. These recommendations include evaluation and clinical and US criteria for cytological biopsy, as well as interpretation of results and management $(6,7)$. Despite this, many patients still undergo diagnostic hemithyroidectomy without prior US and FNAB, and particularly without US of neck lymph nodes. Some of these patients turn out to have PTC NX disease.

Due to the technical challenges and higher risk of complications frequently experienced with repeated neck surgery, this should be kept strictly at a minimum. Hence, the initial preoperative staging should be in agreement with the above-mentioned recommendations. Therefore, central neck lymph node dissection should be performed in conjunction with the thyroidectomy. Thus, a state-of-theart preoperative examination should always include a thorough US examination of the neck performed by an experienced radiologist using high-end US equipment. FNAB from neck lymph nodes should always be performed by an experienced cytopathologist in combination with FNAB-Tg. Surgical treatment becomes appropriate when using this approach, and even more patients may be cured at the initial treatment (21).

As previously reported from our group (10), FNAB-Tg combined with on-site cytological examination improves the sensitivity in detecting metastatic lymph nodes. In this study, FNAB-Tg was conclusive for malignancy in 18 of 109 cases with inconclusive FNAB. Many patients with metastatic disease were detected after 2003 because of more aggressive use of US during follow-up.
Controversy exists regarding prophylactic lymph node resection in levels VI and VII when no metastatic lymph nodes are detected before primary surgery. Irrespective of procedure, some investigators have reported residual or recurrent metastatic neck lymph nodes to occur in $9-20 \%$ of PTC patients during long-term follow-up $(4,5)$.

Due to the indolent biology of PTC, many researchers claim that surgical neck exploration including resection of benign lymph nodes detected by US is not needed (http://cumc.columbia.edu/dept/thyroid/papillary.html) (22). Thus "watch and wait" treatment is often recommended. Lateral cervical lymphadenectomy is mainly performed on patients with verified PTC metastases to lateral neck compartments. Some centers favor a modified radical neck dissection followed by "berry picking" surgery when additional metastases are detected at follow-up (23). PEI treatment of PTC metastases has been used as an alternative to surgery (15). This approach seems to be safe, effective, fast, and without significant complications. Whether one should perform "berry picking" surgery subsequent to modified lymph node dissection or "berry picking" without previous surgery is still under debate (24-26).

PTC patients with undetectable S-Tg (without S-TgAb) and negative US are considered cured. Despite undetectable S-Tg using a sensitive Tg assay, 13 patients without detectable S-TgAb had metastatic neck lymph nodes. Most of these received $\mathrm{T}_{4}$ suppression treatment, and the patients had suppressed TSH values. Stimulated (recombinant human TSH or $\mathrm{T}_{4}$ withdrawal) S-Tg measurement might have disclosed the metastatic disease. This was not performed in these patients. The 30 patients with negative S-TgAb and elevated S-Tg values before PEI and undetectable S-Tg values at end of follow-up did not receive recombinant human TSH stimulation. PEI treatment may cure PTC neck lymph node metastases, but it is still not possible to guarantee that the patient is free from metastatic disease. Indeed, some patients included in our study were diagnosed with recurrent lymph node metastases in other levels after ending the study. The interval from initial surgery to detection of neck lymph node recurrence was highly variable, ranging from 6 to 384 months.

The increasing sensitivity of modern US has resulted in the detection of more and smaller metastatic lymph nodes. Small lymph nodes may be beyond surgical reach. However, in our hospital we have managed to perform PEI treatment on metastatic lymph nodes as small as $2.3 \mathrm{~mm}$. Whether these small lymph node metastases are of clinical importance needs further study. The present results are promising, indicating that US-guided PEI treatment is cost-effective without major side effects and might replace repeated surgical resections. Centers that have adopted a multimodal approach to treat PTC 
report that the crucial success depends on experience and skill. At NRH, we have adopted a multidisciplinary approach to the management of thyroid cancer. Weekly conferences are held with key members of the team, including thyroid surgeons, radiologists, endocrinologists, oncologists, and cytopathologists.

This collaborative, supportive, multidisciplinary team approach has been the key to our success and is a prerequisite for PEI treatment of patients with PTC and neck lymph node metastases.

\section{Acknowledgments}

Address all correspondence and requests for reprints to: Trine Bjøro, Department of Medical Biochemistry, Division of Diagnostics and Intervention, Norwegian Radium Hospital, Oslo University Hospital HF, Ullernchausseen 70, 0310 Oslo, Norway.E-mail: trine.bjoro@medisin.uio.no.

Disclosure Summary: The authors have nothing to disclose.

\section{References}

1. Greenlee RT, Hill-Harmon MB, Murray T, Thun M 2001 Cancer statistics, 2001. CA Cancer J Clin 51:15-36

2. 2010 National Cancer Institute. Surveillance, epidemiology and end results: SEER cancer statistics review 1973-1997. http://seer.cancer. gov/publications

3. Hundahl SA, Fleming ID, Fremgen AM, Menck HR 1998 A National Cancer Data Base report on 53,856 cases of thyroid carcinoma treated in the U.S., 1985-1995 [see comments]. Cancer 83: 2638-2648

4. Hay ID 1990 Papillary thyroid carcinoma. Endocrinol Metab Clin North Am 19:545-576

5. Hay ID, Bergstralh EJ, Grant CS, McIver B, Thompson GB, van Heerden JA, Goellner JR 1999 Impact of primary surgery on outcome in 300 patients with pathologic tumor-node-metastasis stage III papillary thyroid carcinoma treated at one institution from 1940 through 1989. Surgery 126:1173-1181; discussion 1181-1182

6. Pacini F, Schlumberger M, Dralle H, Elisei R, Smit JW, Wiersinga W 2006 European consensus for the management of patients with differentiated thyroid carcinoma of the follicular epithelium. Eur J Endocrinol 154:787-803

7. Cooper DS, Doherty GM, Haugen BR, Hauger BR, Kloos RT, Lee SL, Mandel SJ, Mazzaferri EL, McIver B, Pacini F, Schlumberger M, Sherman SI, Steward DL, Tuttle RM 2009 Revised American Thyroid Association management guidelines for patients with thyroid nodules and differentiated thyroid cancer. Thyroid 19:1167-1214

8. Maxon 3rd HR, Englaro EE, Thomas SR, Hertzberg VS, Hinnefeld JD, Chen LS, Smith H, Cummings D, Aden MD 1992 Radioiodine131 therapy for well-differentiated thyroid cancer-a quantitative radiation dosimetric approach: outcome and validation in $85 \mathrm{pa}-$ tients. J Nucl Med 33:1132-1136

9. Samaan NA, Schultz PN, Hickey RC, Goepfert H, Haynie TP, John- ston DA, Ordonez NG 1992 The results of various modalities of treatment of well differentiated thyroid carcinomas: a retrospective review of 1599 patients. J Clin Endocrinol Metab 75:714-720

10. Sigstad E, Heilo A, Paus E, Holgersen K, Grøholt KK, Jørgensen LH, Bogsrud TV, Berner A, Bjøro T 2007 The usefulness of detecting thyroglobulin in fine-needle aspirates from patients with neck lesions using a sensitive thyroglobulin assay. Diagn Cytopathol 35: 761-767

11. Del Prete S, Caraglia M, Russo D, Vitale G, Giuberti G, Marra M, D’Alessandro AM, Lupoli G, Addeo R, Facchini G, Rossiello R, Abbruzzese A, Capasso E 2002 Percutaneous ethanol injection efficacy in the treatment of large symptomatic thyroid cystic nodules: ten-year follow-up of a large series. Thyroid 12:815-821

12. Sung JY, Baek JH, Kim YS, Jeong HJ, Kwak MS, Lee D, Moon WJ 2008 One-step ethanol ablation of viscous cystic thyroid nodules. AJR Am J Roentgenol 191:1730-1733

13. Livraghi T, Paracchi A, Ferrari C, Reschini E, Macchi RM, Bonifacino A 1994 Treatment of autonomous thyroid nodules with percutaneous ethanol injection: 4-year experience. Radiology 190: 529-533

14. Veldman MW, Reading CC, Farrell MA, Mullan BP, Wermers RA, Grant CS, Thompson GB 2008 Percutaneous parathyroid ethanol ablation in patients with multiple endocrine neoplasia type 1. AJR Am J Roentgenol 191:1740-1744

15. Lewis BD, Hay ID, Charboneau JW, McIver B, Reading CC, Goellner JR 2002 Percutaneous ethanol injection for treatment of cervical lymph node metastases in patients with papillary thyroid carcinoma. AJR Am J Roentgenol 178:699-704

16. Kim BM, Kim MJ, Kim EK, Park SI, Park CS, Chung WY 2008 Controlling recurrent papillary thyroid carcinoma in the neck by ultrasonography-guided percutaneous ethanol injection. Eur Radiol 18:835-842

17. Lim CY, Yun JS, Lee J, Nam KH, Chung WY, Park CS 2007 Percutaneous ethanol injection therapy for locally recurrent papillary thyroid carcinoma. Thyroid 17:347-350

18. Monchik JM, Donatini G, Iannuccilli J, Dupuy DE 2006 Radiofrequency ablation and percutaneous ethanol injection treatment for recurrent local and distant well-differentiated thyroid carcinoma. Ann Surg 244:296-304

19. Cibas ES, Ali SZ 2009 The Bethesda System for Reporting Thyroid Cytopathology. Thyroid 19:1159-1165

20. Edge SB, Byrd DR, Compton CC, Fritz AG, Greene FL, Trotti AE 2010 AJCC cancer staging handbook. 7th ed. New York: Springer

21. Grubbs EG, Evans DB 2007 Role of lymph node dissection in primary surgery for thyroid cancer. J Natl Compr Canc Netw 5:623630

22. Shen WT, Ogawa L, Ruan D, Suh I, Duh QY, Clark OH 2010 Central neck lymph node dissection for papillary thyroid cancer: the reliability of surgeon judgment in predicting which patients will benefit. Surgery 148:398-403

23. Caron NR, Clark OH 2005 Papillary thyroid cancer: surgical management of lymph node metastases. Curr Treat Options Oncol 6:311-322

24. Bhattacharyya N 2003 Surgical treatment of cervical nodal metastases in patients with papillary thyroid carcinoma. Arch Otolaryngol Head Neck Surg 129:1101-1104

25. Musacchio MJ, Kim AW, Vijungco JD, Prinz RA 2003 Greater local recurrence occurs with "berry picking" than neck dissection in thyroid cancer. Am Surg 69:191-196; discussion 196-197

26. Shaha AR 2004 Prognostic factors in papillary thyroid carcinoma and implications of large nodal metastasis. Surgery 135:237-239 


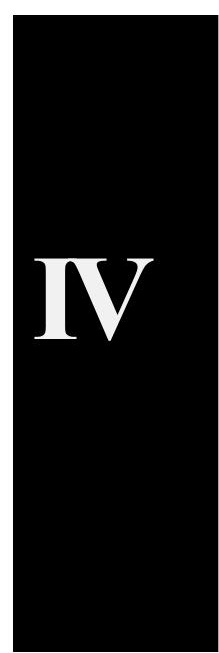





\title{
The new molecular markers DDIT3, STT3A, ARG2 and FAM129A are not useful in diagnosing thyroid follicular tumors
}

\author{
Eva Sigstad ${ }^{1}$, Elisabeth Paus ${ }^{2}$, Trine Bjøro ${ }^{2,3}$, Aasmund Berner ${ }^{1,3}$, \\ Krystyna Kotanska Grøholt ${ }^{1}$, Lars H Jørgensen ${ }^{4}$, Manuel Sobrinho-Simões ${ }^{5}$, \\ Ruth Holm ${ }^{1}$ and David J Warren ${ }^{2}$

\begin{abstract}
${ }^{1}$ Department of Pathology, Division of Diagnostics and Intervention, Oslo University Hospital HF, Montebello, Norway; ${ }^{2}$ Department of Medical Biochemistry, Division of Diagnostics and Intervention, Oslo University Hospital HF, Montebello, Norway; ${ }^{3}$ Department of Medicine, University of Oslo, Oslo, Norway; ${ }^{4}$ Department of Thoracic Surgery, Division of Cardiovascular and Pulmonary Diseases, Oslo University Hospital HF, Montebello, Norway and ${ }^{5}$ IPATIMUP-Institute of Molecular Pathology and Immunology, University of Porto, Porto, Portugal
\end{abstract}

\begin{abstract}
Preoperative characterization of thyroid follicular lesions is challenging. Fine-needle aspiration specimens cannot differentiate follicular carcinomas from benign follicular neoplasias. Recently, promising markers have been detected using modern molecular techniques. We conducted a retrospective study to confirm the usefulness of immunohistochemical staining for the protein markers, DDIT3, STT3A (ITM1), ARG2 and FAM129A (C1orf24) in separating benign and malignant thyroid follicular lesions. Formalin-fixed, paraffin-embedded thyroid tissue from 30 in-house cases (15 follicular carcinomas and 15 follicular adenomas), as well as 8 follicular carcinomas and 21 follicular adenomas on tissue microarray slides were stained immunohistochemically for DDIT3, STT3A, ARG2 and FAM129A expression. Control tissue consisted of thyroid parenchyma adjacent to the tumors and 11 separate cases of normal thyroid parenchyma. All in-house cases of follicular adenomas, follicular carcinomas and adjacent normal thyroid tissue showed positive immunostaining with anti-DDIT3 and anti-STT3A. Anti-ARG2 and anti-FAM129A polyclonal antibodies showed positive staining in 20 and $60 \%$ of in-house follicular adenomas, and 40 and $87 \%$ of in-house follicular carcinomas, respectively. Monoclonal anti-FAM129A demonstrated positive staining in 13 and $33 \%$ of in-house follicular adenomas and follicular carcinomas, respectively. Polyclonal antiDDIT3, -STT3A and -FAM129A antibodies showed positive staining in all tissue microarray slides of follicular carcinoma and in 76,85 and $81 \%$ of the follicular adenomas, respectively. Monoclonal anti-STT3A stained $81 \%$ of the follicular adenoma cores. Anti-ARG2 stained positive in 13\% of follicular carcinomas and $10 \%$ of follicular adenomas on the tissue microarray slides. In conclusion, DDIT3, STT3A, ARG2 and FAM129A immunohistochemistry does not appear to be useful in the diagnosis of thyroid follicular neoplasias, as they do not reliably distinguish follicular thyroid carcinoma from follicular thyroid adenoma.

Modern Pathology (2012) 25, 537-547; doi:10.1038/modpathol.2011.188; published online 9 December 2011
\end{abstract}

Keywords: ARG2; C1orf24; DDIT3; FAM129A; ITM1; STT3A; thyroid carcinoma

Fine-needle aspiration biopsy has become the most widely used preoperative method to diagnose

Correspondence: Dr E Sigstad, MD, Department of Pathology, Division of Diagnostics and Intervention, Oslo University Hospital HF, Montebello, 0310 Oslo, Norway.

E-mail: ESP@ous-hf.no

Received 21 June 2011; revised 29 September 2011; accepted 29 September 2011; published online 9 December 2011 thyroid nodules. ${ }^{1-3}$ Since it's introduction in Europe in the 1970s, and in the US some years later, there has been a dramatic reduction in the need for diagnostic hemi-thyroidectomies, whereas the yield of thyroid carcinomas has increased. ${ }^{4}$ For papillary carcinomas, cytology smears usually are sufficient to make a diagnosis, due to the presence of cells with nuclei characteristic for papillary carcinoma. In marked contrast, in follicular neoplasias, cytological 
morphology cannot reliably distinguish between benign follicular adenomas and malignant follicular carcinomas. ${ }^{5-10}$ Indeed, a definite diagnosis of follicular carcinoma requires surgical excision for histological characterization and the examination of multiple paraffin sections for evidence of capsular or vascular invasion. The reported rate of malignancy in follicular-patterned lesions diagnosed as either atypical or indeterminate ranges between 20 and $30 \%,,^{5,8,11-22}$ and many patients are operated upon unnecessarily. The cost of these procedures is high, both economically and in patient morbidity.

Recently, a number of promising molecular and immunohistochemical methods have been described, which could improve the diagnostic accuracy of fine-needle aspiration biopsy. ${ }^{23-29}$ However, there is some inter-laboratory variability in the sensitivity and specificities obtained with the individual techniques as shown in the review by Griffith et al. ${ }^{30}$ Significantly, none of the methods has been fully validated to the extent that it is in general use in the routine diagnostic laboratory.

In 2006, Cerutti et $a l^{31}$ described a test that could discriminate between follicular carcinoma and follicular adenoma on both paraffin sections and cytology preparations. Their original immunohistochemical method was based on the selective staining of follicular carcinoma cells by antibodies reactive to four protein markers: DDIT3 (also known as CHOP and GADD153), STT3A (also known as ITM1), ARG2 and FAM129A (also known as C1orf24 and Niban). Overexpression of these proteins was first observed using serial analysis of gene expression procedures on a single follicular carcinoma specimen and subsequently confirmed using quantitative reverse-transcription PCR. The four proteins have diverse cellular functions including nitric oxide and polyamine metabolism (ARG2), regulation of protein translation (FAM129A), regulation of transcription as CCAAT/enhancer-binding transcription factor (DDIT3), and as component of the $\mathrm{N}$-oligosaccharide transfer enzyme (STT3A). Cerutti et $a l^{13,32}$ found, by using immunohistochemistry, that the combination of the four markers distinguished a variety of thyroid tumors commonly classified as indeterminate on fine-needle aspiration biopsy, with an estimated sensitivity of $100 \%$ and specificity of $85 \%$ for detecting malignancy.

The aim of this study was to verify the predictive accuracy of the new biomarkers DDIT3, STT3A, ARG2 and FAM129A in distinguishing follicular carcinoma from follicular adenoma.

\section{Materials and methods}

\section{Tissue Sections}

Formalin-fixed, paraffin-embedded thyroid tissue blocks were retrieved from the archive maintained at the Department of Pathology, The Norwegian Radium Hospital. A total of 30 cases, consisting of
15 follicular carcinomas and 15 follicular adenomas, originally submitted for diagnostic purposes, were included. The in-house cases have been diagnosed histopathologically according to World Health Organization criteria. ${ }^{33}$ Follicular neoplasias with oncocytic differentiation, and areas containing cells demonstrating oncocytic differentiation, were excluded from the evaluation. In all cases, hematoxylin and eosin-stained slides were reviewed to confirm the original histopathological diagnosis. Before embedding, the tissue specimens had been preserved in $4 \%$ buffered formalin for variable periods, but within the range of 2-5 days.

Two tissue microarrays containing either 80 samples from normal thyroid parenchyma (serial TH804) or 80 thyroid cases with pathological findings, including 8 follicular carcinomas and 21 follicular adenomas (serial TH802), were purchased from US Biomax (Rockville, USA). The product supplier stated that the tissues used to construct these arrays had been preserved in neutral phosphate-buffered formalin for a maximum of $24 \mathrm{~h}$ before embedding.

\section{Antibody Preparation}

Rabbit polyclonal antisera to FAM129A (C1orf24) and STT3A (ITM1) were generated using C-terminal peptides as described by Cerutti et al. ${ }^{31}$ The peptides were custom-synthesized and conjugated to diphtheria toxoid (Mimotopes, Clayton, VIC, Australia). Immunizations were performed using the diphtheria toxoid conjugates by Eurogentec (Liège, Belgium). Monoclonal antibodies were produced by fusion of NSo myeloma cells with splenocytes from BALB/c mice immunized with peptide-diphtheria toxoid conjugates.

Antibody titration and hybridoma screening were performed using antibody capture assays with biotinylated peptides presented on streptavidin-coated microplates (Wallac Oy, Turku, Finland). Specificity was established using ELISA, western blotting and ${ }^{125}$ I-peptide displacement assays.

Polyclonal rabbit anti-GADD 153 (DDIT3; sc-793) and anti-Arginase-2 (sc-20151) were obtained from Santa Cruz Biotechnology (Santa Cruz, CA, USA).

Experimental animals were handled in accordance with national and institutional guidelines.

\section{${ }^{125}$ I-Peptide Displacement Assays}

Competitive immunoassay tracers were produced by incubating biotinylated peptides with an excess of ${ }^{125}$ I-labeled streptavidin. Iodination was performed with the indirect Iodogen-method (Pierce, Rockford, IL, USA). The test was performed by incubating tracer and antisera (dilution 1:10000) for $3 \mathrm{~h}$ in the presence and absence of tissue homogenate. Antibody/antigen complexes were then isolated by the addition of sheep anti-immunoglobulin-coated 
magnetic beads (Invitrogen, Carlsbad, CA, USA). The suspension was incubated for $1 \mathrm{~h}$ with shaking, washed three times and bound tracer counted in a $\gamma$-counter.

\section{Western Blotting}

Tissue samples or cells from a follicular carcinoma line, FTC-133 (EACC, Salisbury, UK) were homogenized in ice-cold RIPA buffer (TBS, 1\% NP-40, $0.25 \%$ sodium deoxycholate, $0.1 \%$ SDS) containing $5 \mathrm{mM}$ EDTA, $1 \mathrm{mM}$ phenylmethylsulfonylfluoride, $0.2 \mu \mathrm{M}$ aprotinin, $1 \mu \mathrm{M}$ leupeptin and $1 \mu \mathrm{M}$ pepstatin A. Following clarification, soluble protein concentrations were determined using the BCA method (Pierce). Between 20 and $40 \mu \mathrm{g} /$ lane of total protein was subjected to SDS-PAGE (Invitrogen; Novex $4-12 \%$ gradient) and subsequently transferred onto nitrocellulose. The membranes were blocked with 5\% skimmed milk/TBS, followed by incubation for $1 \mathrm{~h}$ with the monoclonal (1:10 dilution of culture supernatant) or polyclonal (1:20001:5000) antibodies diluted in 5\% milk/TBS. Loading controls were stained for GAPDH and $\alpha$-tubulin. Detection utilized HRP-labeled goat anti-species immunoglobulin (1:6000 dilution poly-HRP, Pierce) and the SuperSignal West Pico chemiluminescent substrate (Pierce).

\section{Immunohistochemical Staining}

Immunohistochemistry was performed on $3 \mu \mathrm{m}$ paraffin sections mounted on positively charged slides, essentially as described by Cerutti et al. ${ }^{31}$ Tissue sections were dewaxed in xylene, followed by rehydration using a graded ethanol series. Endogenous peroxidase activity was suppressed by pretreatment with $0.03 \%$ hydrogen peroxide, and antigen retrieval was achieved using buffer AR-10 (Biogenex, San Ramon, CA, USA) in a steamer for 10 min. Immunodetection was undertaken using a Dako automated tissue-staining system (S3400; Dako, Carpinteria, CA, USA), using EnVision+ HRP reagents (K4007 and K4011, Dako). Tissue sections were exposed to the primary antibodies for $20-22 \mathrm{~h}$ at $4{ }^{\circ} \mathrm{C}$. The optimal dilution of each antibody was determined in preliminary staining trials. Appropriate controls were prepared using (1) non-immune polyclonal rabbit antisera or mouse IgG1 myeloma immunoglobulin and (2) antiFAM129A and anti-STT3A, preabsorbed with $1 \mu \mathrm{g} / \mathrm{ml}$ FAM129A antigen and $1 \mu \mathrm{g} / \mathrm{ml}$ STT3A antigen, respectively.

Two independent surgical pathologists (ES and $\mathrm{AaB}$ ) performed the slide evaluation. Tissue sections were assigned to one of four groups, based on the proportion of cells showing unequivocal staining: 0, (0\%); 1, (<25\%); 2, (25-50\%); 3, (51-75\%); 4 , $(>75 \%)$. The scoring system used a cutoff for positive staining of $>25 \%$, which is in accordance with accepted scoring methods. Arginase-2 and STT3A staining was detected primarily in the cytoplasm of the follicular cells, FAM129A in the cytoplasmic membrane and the cytoplasm, whereas DDIT3 expression was observed both in the nucleus and the cytoplasm. For each of the in-house cases, staining of the normal thyroid tissue adjacent to the tumor was also evaluated. The inter-observer agreement was $94 \%$ for the in-house cases and $83 \%$ for the tissue microarray slides. Discordant cases were reevaluated using a multi-headed microscope to obtain a consensus. Images displayed in this article were captured using a Leica DMLB microscope (Type 020-519.511DMLB 100T; Leica Microsystems $\mathrm{GmbH}$, Germany) equipped with a Nikon digital Sight DS-Fi1 camera (Nikon Corporation, Japan), and processed using JASC Paint Shop Pro software (Version 7.02, Corel Corporation, USA).

\section{Statistics}

The data were analyzed using the Statistical Package for the Social Sciences (SPSS), version 17.0.1 for Windows (SPSS, Chicago, IL, USA). The Mann-Whitney $U$-test was used to determine DDIT3, STT3A, ARG2 and FAM129A expression level differences between follicular adenomas and follicular carcinomas. A $P$-value of $<0.05$ was considered statistically significant. Sensitivity and specificity were calculated using standard formulae for each of the antibodies individually, using benign versus malignant histological diagnosis as the standard.

\section{Ethics}

The study was approved by the Regional Committee for Medical Research Ethics and the 'Personvernombudet' at Rikshospitalet. Informed consent for the use of material was obtained from the patients.

\section{Results}

\section{Production and Characterization of Antibodies}

Antibodies to STT3A and FAM129A are not commercially available and were produced in-house. Rabbits were hyper-immunized with the appropriate C-terminal peptide and antibody titers followed by antibody-capture ELISA. After one primary injection and three booster doses, antisera with titers $>1: 10000$ were obtained. The sera were screened by radioimmunoassay displacement analysis using follicular carcinoma, follicular adenoma and normal tissue lysates. Sera that demonstrated more efficient peptide displacement with follicular carcinoma lysates compared with follicular adenoma or normal cell extracts were selected (data not shown). The specificity of the anti-FAM129A and anti-STT3A antibodies determined by western 
blotting of total cell lysates of the FTC-133 cell line detected proteins with the expected molecular weight of approximately 130 and $60 \mathrm{kDa}$, respectively (Figure 1).

Monoclonal antibodies were also generated using splenocytes from mice immunized with the peptide conjugates. Approximately, 1000 parental hybridomas/peptide were screened for reactivity by antibody-capture assay using biotinylated peptides. Secondary screening was undertaken by immunocytochemistry using the FTC-133 cell line. Two hybridomas were selected, producing antibodies to FAM129A (clone E253) and STT3A (clone E239).

The specificity of the anti-FAM129A and antiSTT3A was determined by preabsorption of the antiserum with blocking peptides. Absorbed polyclonal and monoclonal anti-FAM129A antibodies and the monoclonal anti-STT3A antibody gave

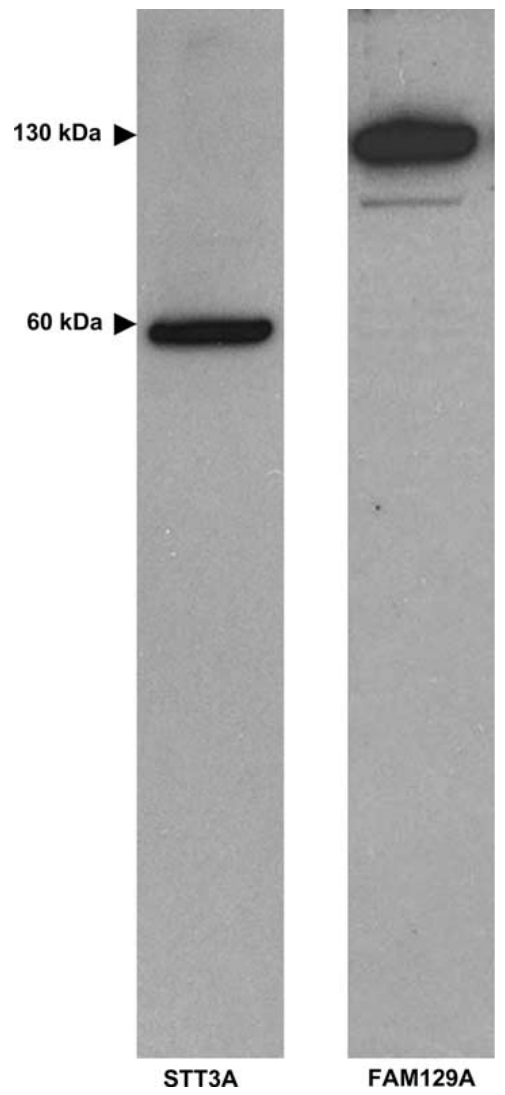

Figure 1 Specificity of the anti-FAM129A and anti-STT3A antibodies as determined by western blotting. Total cell lysates of the FTC-133 cell line were subjected to SDS-PAGE using 4$12 \%$ gradient gels. After transfer, the nitrocellulose membranes were stained with the primary antibody followed by a HRP-goat anti-rabbit secondary reagent. Detection utilized a chemoluminescent substrate. negative immunostaining. A faint immunoreaction was observed with preabsorbed polyclonal antiSTT3A antibody (data not shown). However, this background was easily distinguishable from a positive staining reaction.

\section{Immunohistochemistry}

Paraffin sections from 30 in-house cases were screened for the expression of DDIT3, STT3A, ARG2 and FAM129A, using a panel of polyclonal and monoclonal antibodies (Figure 2a). Positive staining for anti-DDIT3 and anti-STT3A was observed in all the cases of follicular carcinoma and follicular adenoma. The normal tissue adjacent to the tumors was also stained. Comparable binding was obtained with polyclonal or monoclonal antiSTT3A antibodies. ARG2 was detected in $40 \%$ of follicular carcinoma cases, $20 \%$ of the follicular
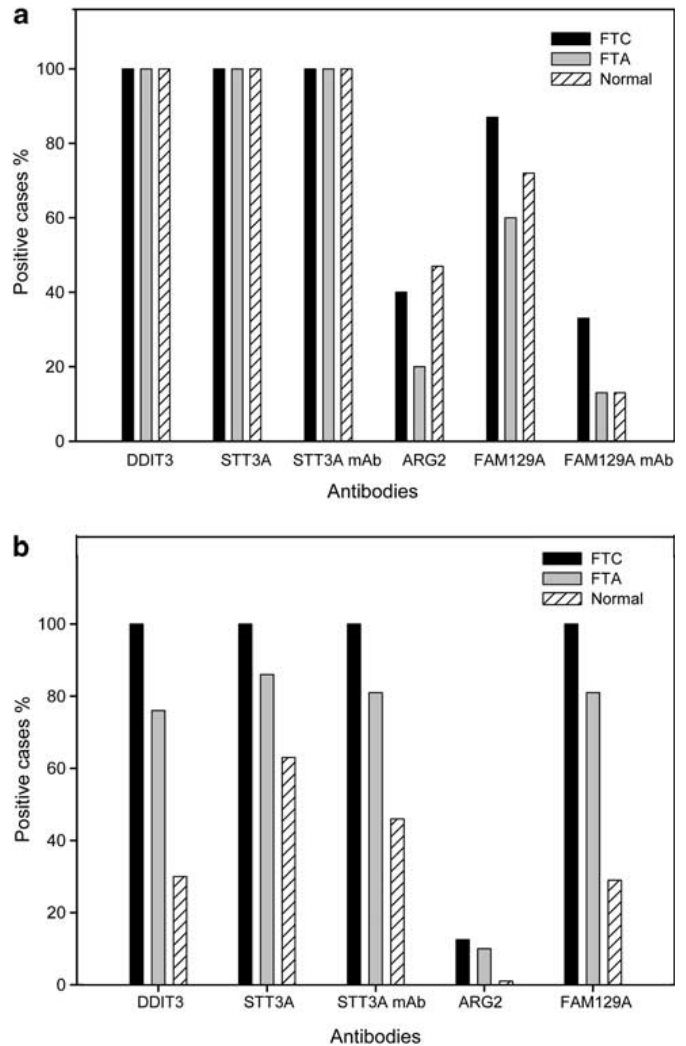

Figure 2 Frequency of binding of the four antibodies to follicular adenoma, follicular carcinoma and adjacent normal thyroid tissue in in-house cases (a), and in follicular adenoma, follicular carcinoma and separate cases of normal thyroid tissue in tissue microarray cases (b). FTA = follicular thyroid adenoma, FTC $=$ follicular thyroid carcinoma, $\mathrm{N}=$ normal thyroid tissue. 
adenomas and $47 \%$ of the normal thyroid tissues adjacent to the tumors. The polyclonal antibody to FAM129A demonstrated positive staining of follicular cells in $87 \%$ of the follicular carcinoma cases, $60 \%$ of the follicular adenomas and $72 \%$ of the adjacent normal tissues. In contrast, the antiFAM129A monoclonal antibody stained 33\% of follicular carcinoma cases, and $13 \%$ of both the follicular adenomas and the adjacent normal tissues. The immunohistochemical staining with the four antibodies of in-house follicular adenoma and follicular carcinoma cases, and the adjacent nonneoplastic thyroid tissue and two negative controls is shown in Figure 3. The observed staining of normal tissue was not due to leaking from the adjacent follicular adenoma or follicular carcinoma, as we found antibody binding in a panel of 11 thyroid specimens that displayed normal morphology. With this normal tissue panel, binding of the polyclonal DDIT3, STT3A and ARG2 antibodies was seen in all 11 individuals. Staining with the FAM129A polyclonal antibody was found in nine cases, whereas the monoclonal anti-FAM129A and anti-STT3A stained seven and nine cases, respectively.

In preliminary trials, we tried to ameliorate what we thought was the 'artifactual' staining of follicular adenoma and normal thyroid parenchyma, using a range of techniques. These included alternative antigen-retrieval buffers (citrate, Tris-Cl, citraconic anhydride), peroxidase blocking $\left(\mathrm{H}_{2} \mathrm{O}_{2}, \mathrm{H}_{2} \mathrm{O}_{2}\right.$ / methanol, $\mathrm{H}_{2} \mathrm{O}_{2}$ /sodium azide), protein blocking reagent (normal serum, casein, fish gelatine, different BSA batches) and pre-treatment of the primary antibodies with thiol reagents. In each case, we failed to block the staining of follicular adenoma and normal thyroid epithelium (data not shown).

On the tissue microarray slides (Figure 2b), expression of DDIT3, STT3A and FAM129A was found in all the follicular carcinoma tissue cores and in 76,85 and $81 \%$ follicular adenoma cores, respectively. Only $13 \%$ follicular carcinoma samples and $10 \%$ of the follicular adenoma cores showed positive staining with anti-ARG2. The tissue microarray slides containing normal thyroid tissue (which did not originate from the same individuals as the tumor cases) showed positive staining in 30,63 and $29 \%$, of the cores with polyclonal anti-DDIT3, -STT3A and -FAM129A, respectively. Expression of ARG2 was detectable in only $1 \%$ of individuals. The monoclonal antibody to STT3A stained $46 \%$ of the tissue cores. Immunostaining with the four antibodies of tissue microarray cores from follicular adenoma and follicular carcinoma cases, and separate tissue microarray cores from normal thyroid tissue is shown in Figure 4.

Mann-Whitney test was performed on the scoring data from both in-house slides and tissue microarray slides. The evaluation of difference in sum of ranks between follicular carcinoma and follicular adenoma cases revealed no significant $P$-values for the in-house cases, and for the tissue microarray cases, a $P$-value of 0.04 for DDIT3, and a non-significant value for STT3A polyclonal of $P=0.37$, for STT3Amonoclonal of $P=0.24$, for ARG2 of $P=0.28$ and for FAM129A of $P=0.08$. Defining sensitivity as the proportion of follicular carcinoma patients who expressed a marker, and specificity as the proportion of patients with follicular adenoma, who did not express the marker, ${ }^{31}$ the sensitivity and specificity of staining with each of the antibodies were calculated, and a summary of the calculated sensitivity and specificity of staining with each of the antibodies for the in-house and tissue microarray slides is shown in Table $1 \mathrm{a}$ and $\mathrm{b}$, respectively.

\section{Tissue Distribution of DDIT3, STT3A, ARG2 and FAM129A by Western Blotting}

The results from immunoblotting studies are shown in Figure 5. Tissue extracts from two carcinomas and five adenomas were used. For one follicular carcinoma (lane 2) and one follicular adenoma (lane 5), tissue extracts from the non-involved lobe were also available for testing (sample lane 3 and lane 4, respectively). Our polyclonal and monoclonal antibodies to STT3A showed high specificity and detected a protein with the expected molecular weight of approximately $60 \mathrm{kDa}$. Immunoblots performed using the polyclonal anti-STT3A or the corresponding monoclonal antibody showed good concordance. With both reagents, STT3A protein was found in all follicular carcinoma cases and in three out of five of the follicular adenoma tissue lysates. Low, but detectable STT3A expression was also found in normal thyroid tissue. The polyclonal anti-FAM129A was highly specific, detecting a protein with an apparent Mw of about $130 \mathrm{kDa}$. FAM129A was highly expressed in one of the follicular carcinoma lysates, four out of five follicular adenoma cases and one normal tissue extract. In contrast to the anti-STT3A monoclonal antibody, the anti-FAM129A monoclonal antibody performed very poorly in western blotting and was not characterized (data not shown). The commercial anti-ARG2 reacted with a protein of $40 \mathrm{kDa}$ with good specificity. The protein was highly expressed in both follicular carcinomas and three out of five follicular adenoma lysates. Low levels of ARG2 were found in all the tissue samples. Surprisingly, the commercial anti-DDIT3 proved highly nonspecific, showing binding to many proteins within the $50-200 \mathrm{kDa}$ range. This antibody did, however, detect a $30 \mathrm{kDa}$ protein, presumably mature DDIT3, in one of the follicular carcinoma, but none of the follicular adenoma tumor lysates.

\section{Discussion}

Fine-needle aspiration biopsy of the thyroid is the most commonly used preoperative method to 

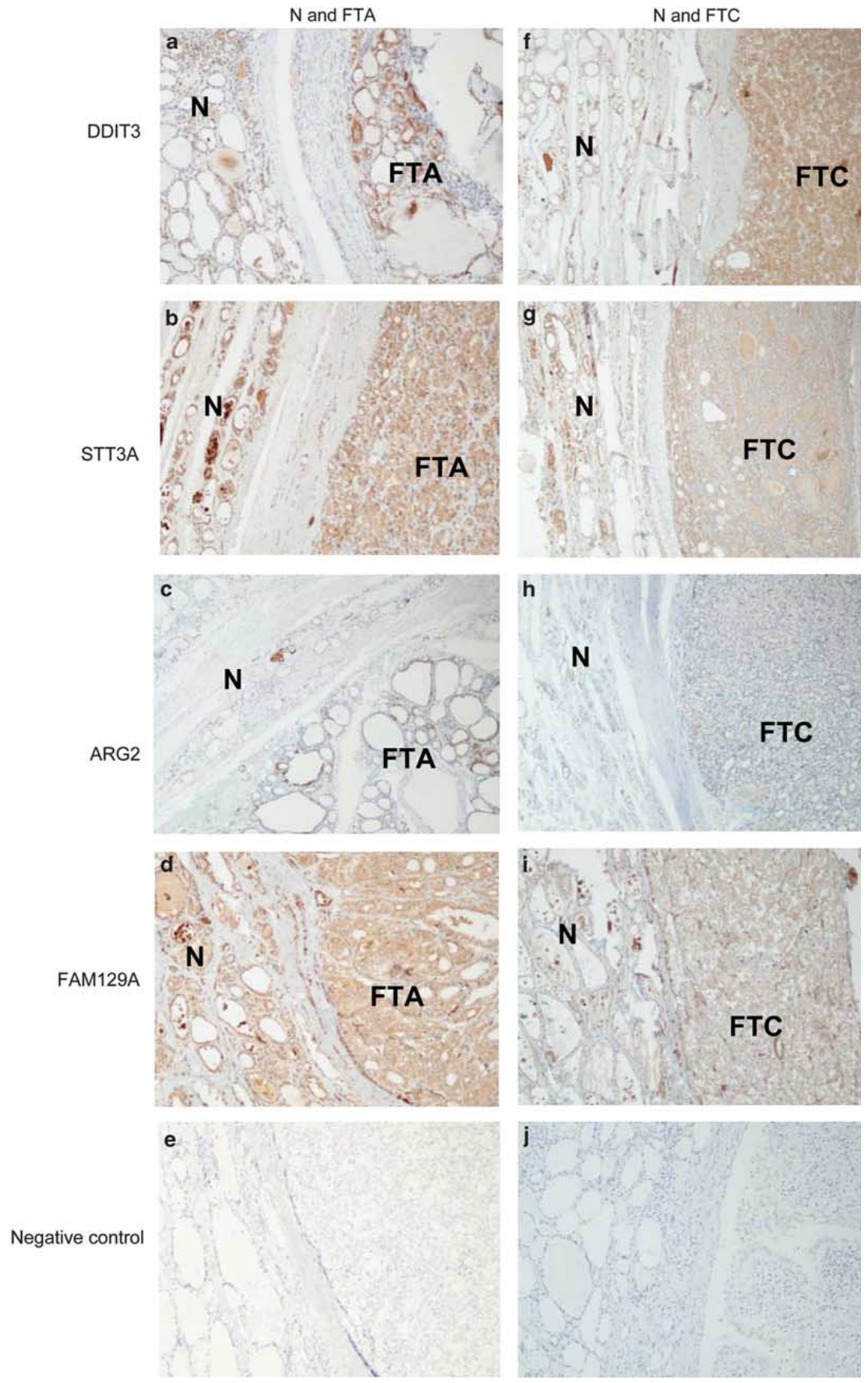

Figure 3 Immunohistochemical detection of DDIT3, STT3A, ARG2 and FAM129A in follicular adenoma and the adjacent normal thyroid tissue $(\mathbf{a}-\mathbf{d} ; \times \mathbf{5 0})$ and follicular carcinoma and the adjacent normal thyroid tissue $(\mathbf{f}-\mathbf{i} ; \times 50)$ in in-house cases. Negative controls are shown in panels $\mathbf{e}$ and $\mathbf{j}$. FTA = follicular thyroid adenoma, FTC = follicular thyroid carcinoma, $\mathrm{N}=$ normal thyroid tissue. 

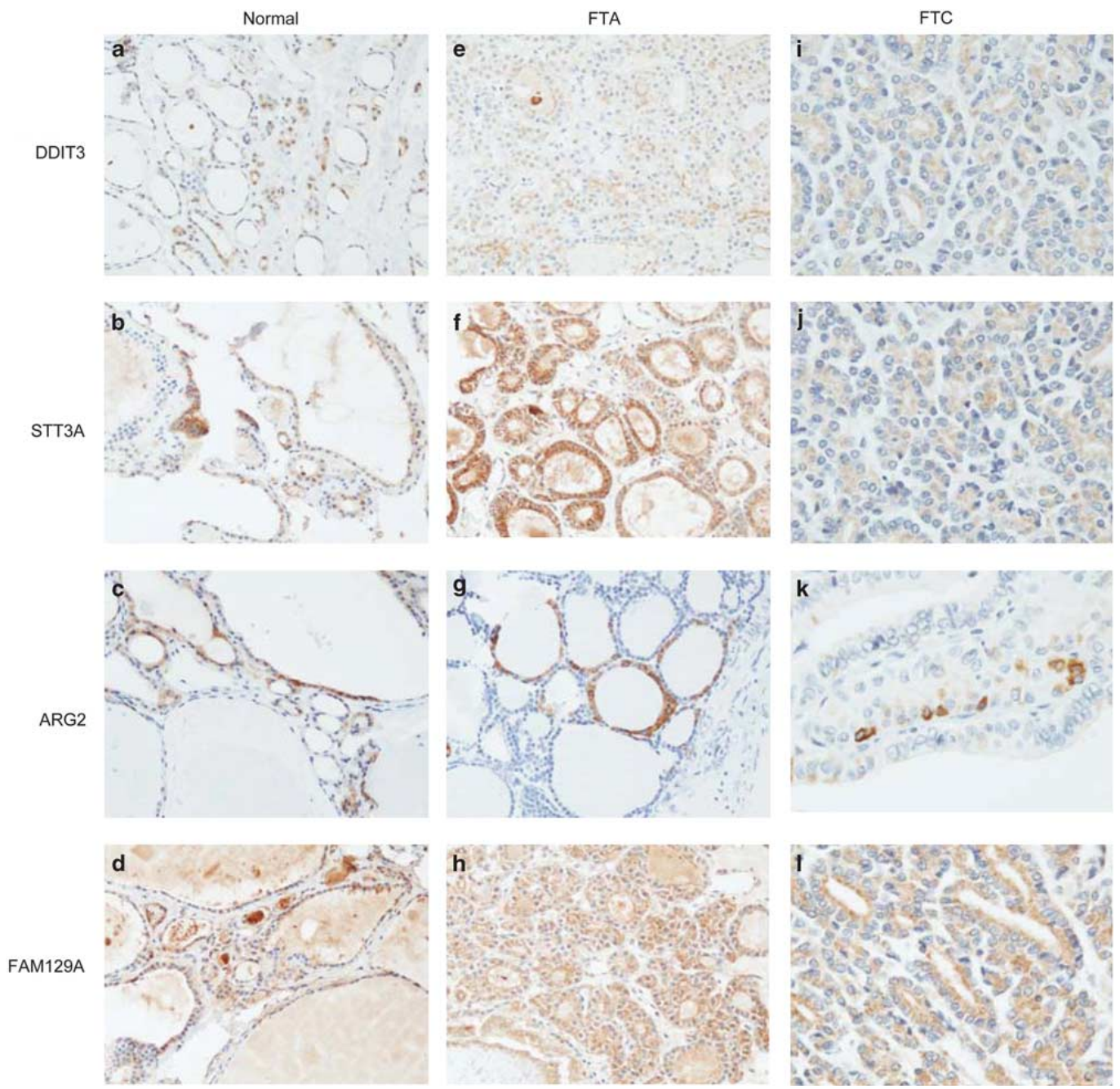

Figure 4 Immunohistochemical detection of DDIT3, STT3A, ARG2 and FAM129A in separate cases of normal thyroid tissue (a-d; $\times 200)$, follicular adenoma $(\mathbf{e}-\mathbf{h} ; \times 200)$ and follicular carcinoma $(\mathbf{i}-\mathbf{l} ; \times 400)$ on tissue microarray slides. FTA $=$ follicular thyroid adenoma, FTC = follicular thyroid carcinoma.

diagnose thyroid tumors. Although fine-needle aspiration biopsy can reduce the number of diagnostic thyroidectomies by identifying clearly benign lesions, it cannot reliably distinguish between follicular adenoma and follicular carcinoma. Many authors have attempted to identify molecular markers able to predict follicular carcinoma, but with conflicting results. ${ }^{30}$ The discordances may be a result of the biased selection of material, the limited number of cases examined, or the use of very different molecular and immunohistochemical methods. Among the most promising markers in the recent literature are DDIT3, STT3A, ARG2 and FAM129A that, according to Cerutti et al, reliably identify follicular carcinoma. However, the utility of these markers has not been confirmed by other laboratories, and they are not widely used in diagnostic practice.

In this study, we tested the ability of immunohistochemical staining for DDIT3, STT3A, ARG2 and FAM129A, to distinguish follicular carcinoma from follicular adenoma. Our intention was to optimize the previously described methodology before establishing it in our diagnostic routine. Surprisingly, and discordant to the results of Cerutti et $a{ }^{31}{ }^{31}$ we found, as shown in Figure 2a, the percentage of our in-house cases with positive staining of follicular adenoma and follicular carcinoma to be identical 
Table 1 Sensitivity and specificity of immunohistochemistry with CI; sensitivity is defined as the proportion of follicular carcinoma patients who expressed the marker in tumor, and specificity as the proportion of patients with follicular adenoma, who did not express the marker ${ }^{31}$

\begin{tabular}{lcccc}
\hline & Sensitivity & 95\% CI & Specificity & 95\% CI \\
\hline (a) In-house cases & & & & \\
DDIT3 & 1.00 & - & 0 & - \\
STT3Ap & 1.00 & - & 0 & - \\
STT3Am & 1.00 & - & 0 & - \\
ARG2 & 0.40 & $0.29-0.64$ & 0.80 & $0.55-0.93$ \\
FAM129Ap & 0.87 & $0.62-0.96$ & 0.40 & $0.20-0.64$ \\
FAM129Am & 0.33 & $0.15-0.58$ & 0.87 & $0.62-0.96$ \\
& & & & \\
(b) Tissue microarray cases & & & 0.24 & $0.11-0.45$ \\
DDIT3 & 1.00 & - & 0.14 & $0.05-0.35$ \\
STT3Ap & 1.00 & - & 0.19 & $0.08-0.40$ \\
STT3Am & 1.00 & - & 0.90 & $0.71-0.97$ \\
ARG2 & 0.12 & $0.02-0.47$ & 0.19 & $0.08-0.40$ \\
FAM129Ap & 1.00 & - & & \\
\hline
\end{tabular}

Abbreviation: CI, confidence intervals.

$(100 \%)$ for two of the antibodies examined (DDIT3 and STT3A), and with no significant difference in percentage of cases of follicular adenoma and follicular carcinoma with staining for ARG2 and FAM129A. Furthermore, the percentage of cases showing staining of the adjacent benign thyroid cells was not significantly different from the percentage of tumor cases showing staining. When examining the tissue microarray cases (Figure 2b), we found no significant difference in percentage of positive follicular adenoma and follicular carcinoma cases for three of the four antibodies examined (STT3A, ARG2 and FAM129A), and only for DDIT3, we did find the difference in percentage of positively stained cases of follicular adenoma and follicular carcinoma to be of weak significance. It is unlikely that the positive DDIT3, STT3A, ARG2 and FAM129A immunostaining of follicular adenoma and normal tissues was due to differences in methodology. We used the same antigen retrieval and staining protocols as originally described by Cerutti et $a l^{31,32}$ and extensively optimalized the dilutions of the primary antibodies used. Furthermore, staining was performed using the same commercially available antibodies against ARG2 and DDIT3 utilized by Cerutti et al, ${ }^{31}$ and in-house generated reagents were found to have high specificity to their cognate antigen in western blots. However, the anti-FAM129A monoclonal antibody performed poorly in immunohistochemistry and western blotting, probably reflecting its low avidity.

We additionally stained multiple cores of follicular adenoma, follicular carcinoma and normal thyroid parenchyma on commercially constructed tissue microarray slides. The tissue microarray results agreed with those obtained using wax sections prepared in-house, and positive follicular cell staining was found in cores containing normal thyroid tissue. The $P$-value of 0.04 for DDIT3 was found examining a small number of follicular carcinoma $(n=8)$ and follicular adenoma $(n=21)$ tissue microarray specimens. This finding does not fit with the observations made of the in-house cases demonstrating positive staining in all follicular adenomas $(n=15)$ and all follicular carcinomas $(n=15)$. Additionally, some quantitative differences were seen when comparing staining with anti-ARG2 and anti-FAM129A polyclonal antibodies on fresh sections with that on tissue microarray slides. These were most probably related to variable degrees of epitope degradation during the prolonged storage of the commercial tissue microarrays. ${ }^{34}$ However, as the tissues used to construct these arrays were fixed, embedded and sectioned by an independent laboratory, they provide an appropriate control for the variables associated with paraffin block preparation and handling.

Because of the surprising disparity between our immunohistochemical results and those described previously, ${ }^{31,32}$ we determined the expression of ARG2, FAM129A, DDIT3 and STT3A in follicular adenoma and follicular carcinoma biopsy specimens, using western blotting. In contrast to immunohistochemistry, western blotting can evaluate protein expression, antibody specificity and provide a $\mathrm{M}_{\mathrm{r}}$ of the antibody-binding moiety and hence, a presumptive identity. With the exception of the commercial anti-DDIT3 antibody, the antibody reagents demonstrated good specificity and reacted with antigens of the expected size in follicular adenoma and follicular carcinoma lysates. In agreement with our observations using immunohistochemistry, a sizable proportion of the follicular adenomas displayed significant expression of ARG2, FAM129A and STT3A. Although anti-DDIT3 (Santa Cruz Biotechnology) detected a protein of the expected size in one follicular carcinoma lysate, it displayed marked nonspecificity. In a recent paper by Haataja et $a l,{ }^{35}$ three out of seven commercially available DDIT3 antibodies (including the antibody from Santa Cruz Biotechnology that we used) gave false results by western blotting and immunocytochemistry. This non-specificity may explain why we observed positive immunohistochemical staining in all freshly cut follicular adenoma and normal tissue sections. Although Cerutti et $a l^{31,32}$ used the same antibody, they did not report on whether they characterized each of their purchased batches.

Considering the urgent need for a technique that can reliably discriminate between follicular carcinoma and follicular adenoma, it is surprising that a search of the literature indicates that we are the first to attempt to replicate a method originally described in 2004. ${ }^{32}$ Matsumoto et $a l^{36}$ observed binding of antibodies to FAM129A to thyroid tumors with oxyphilic cytoplasm, to oxyphilic follicular adenoma and to oxyphilic variants of follicular carcinoma. However, the authors also noted FAM129A expression in $25 \%$ of follicular non-oxyphilic follicular adenoma and in only $50 \%$ of the non-oxyphilic 

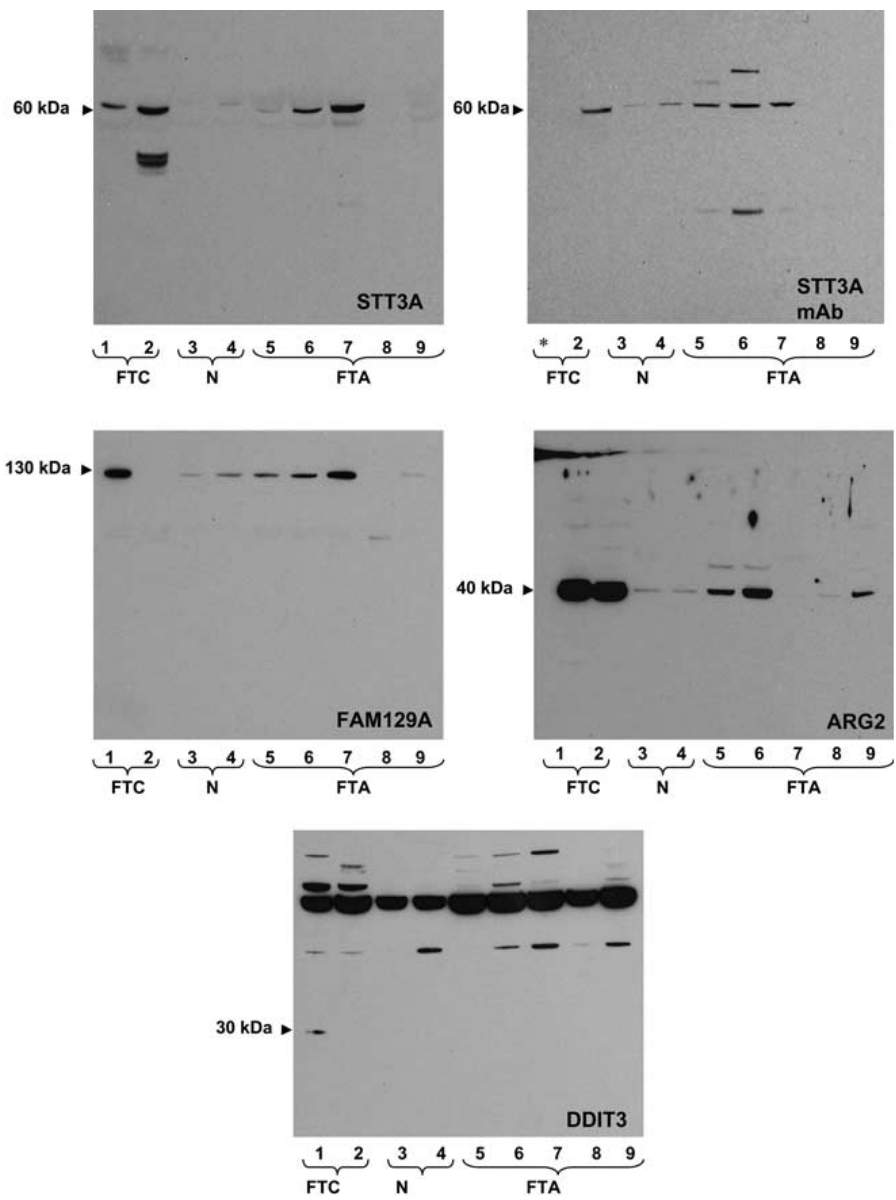

Figure 5 Determination of ARG2, FAM129A, DDIT3 and STT3A tissue expression by western blotting. Lysates prepared from follicular carcinoma (lane 1,2), normal thyroid parenchyma (lane 3,4) and follicular adenoma (lane 5-9) tissue were subjected to western blotting as described in the legend to Figure 1. Normal tissues 2 and 3 were obtained from the uninvolved thyroid lobe of patent 2 and 5 , respectively. ${ }^{*}$ Sample not run due to insufficient lysate.

follicular carcinomas. This study would therefore also suggest that FAM129A expression discriminates poorly between follicular carcinoma and follicular adenoma. In a recent reverse-transcription PCR study by Patel et $a{ }^{37}$ on fine-needle aspiration biopsy specimens, a marked downregulation of FAM129A was found in follicular carcinoma, follicular variant of papillary thyroid carcinoma and oxyphilic follicular carcinomas, compared with matched-normal tissue. The findings by Matsumoto et $a l^{36}$ and Patel et $a l^{37}$ are discordant to those presented by Cerutti et $a l^{38}$ in a recent validation study, which confirmed their previous findings of overexpression of FAM129A in all follicular carcinomas. Bryson et al ${ }^{39}$ found significant differences in DDIT3 expression in a tissue panel comprising 62 follicular adenomas and 62 follicular carci- nomas. However, they determined a sensitivity of $82 \%$ with a specificity of only $21 \%$, which is in agreement with our results. A study by Netea-Maier et $a l^{40}$ using different gel electrophoresis found no variance in the abundance of ARG2, FAM129A, DDIT3 and STT3A between follicular carcinoma and follicular adenoma. The authors concluded that the differences in mRNA levels between follicular carcinoma and follicular adenoma as reported by Cerutti et $a l^{32}$ are of limited value, because mRNA expression frequently does not reflect protein abundance. Similarly, a study performing global gene expression analysis of follicular thyroid tumors found no difference in ARG2, DDIT3 and STT3A expression between follicular adenoma and follicular carcinoma ${ }^{41}$ In a meta-analysis and meta-review by Griffith et $a l^{42}$ on 21 published thyroid cancer 
gene expression studies, including the study by Cerutti et al, ${ }^{32}$ ARG2, DDIT3, STT3A and FAM129A are not among the 39 markers with overlap of three or more candidate markers for the cancer versus non-cancer group.

Our insight into gene expression in tumor cells has increased rapidly with the help of DNA and protein microarray analyses. However, the interpretation of the acquired data is challenging, and is subject to several potential pitfalls. The importance of a close collaboration between the investigators performing the microarrays and experienced biostatisticians is crucial. ${ }^{43}$ Morphology is still the gold standard when evaluating thyroid neoplasms. Indeed, for the majority of thyroid tumors, the identification of "abnormal" tissue on histological preparations will probably long remain a prerequisite before performing immunohistochemistry or molecular analysis. Unfortunately, as this study demonstrates, the search for the ideal marker capable of discriminating between follicular carcinoma and follicular adenoma must continue.

In conclusion, in this study, we determined whether screening for the expression of the biomarkers DDIT3, STT3A, ARG2 and FAM129A, is able to distinguish between follicular thyroid adenoma and carcinoma. Regrettably, using both immunohistochemistry and western blotting, we demonstrate that these markers are not useful for this purpose. The reasons for the discordance between our results and those of Cerutti et $a l^{31,32}$ are, at present, and despite considerable focus on quality assurance of all steps, unknown. In our hands, the method is not usable for screening thyroid nodules in patient populations. The failure of this study to replicate a previously published method highlights the need for extensive validation studies to determine if a previously advanced classifier has clinical utility in an independent set of cases.

\section{Acknowledgements}

We deeply appreciate and thank Ms Ellen Hellesylt for her great work in the tissue processing and staining. This study was supported by Grant from the Norwegian Cancer Society and South-Eastern Norway Regional Health Authority, project no 2009008.

\section{Disclosure/conflict of interest}

The authors declare no conflict of interest.

\section{References}

1 Hegedus L. Clinical practice. The thyroid nodule. N Engl J Med 2004;351:1764-1771.

2 Gharib H, Goellner JR. Fine-needle aspiration biopsy of the thyroid: an appraisal. Ann Intern Med 1993;118: $282-289$.
3 Suen KC. Fine-needle aspiration biopsy of the thyroid. CMAJ 2002;167:491-495.

4 Hamberger B, Gharib H, Melton III LJ, et al. Fineneedle aspiration biopsy of thyroid nodules. Impact on thyroid practice and cost of care. Am J Med 1982;73: 381-384.

5 Fadda G, Rabitti C, Minimo C, et al. Morphologic and planimetric diagnosis of follicular thyroid lesions on fine needle aspiration cytology. Anal Quant Cytol Histol 1995;17:247-256.

6 Fadda G, Balsamo G, Fiorino MC, et al. Follicular thyroid lesions and risk of malignancy: a new diagnostic classification on fine-needle aspiration cytology. J Exp Clin Cancer Res 1998;17:103-107.

7 Baloch ZW, Fleisher S, Livolsi VA, et al. Diagnosis of 'follicular neoplasm': a gray zone in thyroid fineneedle aspiration cytology. Diagn Cytopathol 2002;26: 41-44.

8 Yang GC, Liebeskind D, Messina AV. Should cytopathologists stop reporting follicular neoplasms on fine-needle aspiration of the thyroid? Cancer 2003;99: 69-74.

9 Yang J, Schnadig V, Logrono R, et al. Fine-needle aspiration of thyroid nodules: a study of 4703 patients with histologic and clinical correlations. Cancer 2007; 111:306-315.

10 Yassa L, Cibas ES, Benson CB, et al. Long-term assessment of a multidisciplinary approach to thyroid nodule diagnostic evaluation. Cancer 2007;111: 508-516.

11 Schlinkert RT, van Heerden JA, Goellner JR, et al. Factors that predict malignant thyroid lesions when fine-needle aspiration is 'suspicious for follicular neoplasm'. Mayo Clin Proc 1997;72:913-916.

12 Goodell WM, Saboorian MH, Ashfaq R. Fine-needle aspiration diagnosis of the follicular variant of papillary carcinoma. Cancer 1998;84:349-354.

13 Tuttle RM, Lemar H, Burch HB. Clinical features associated with an increased risk of thyroid malignancy in patients with follicular neoplasia by fineneedle aspiration. Thyroid 1998;8:377-383.

14 Carpi A, Menchini FF, Ferrari E, et al. Aspiration needle biopsy in preoperative selection of thyroid nodules defined at fine-needle aspiration as microfollicular lesions. Am J Clin Oncol 1999;22:65-69.

15 Raber W, Kaserer K, Niederle B, et al. Risk factors for malignancy of thyroid nodules initially identified as follicular neoplasia by fine-needle aspiration: results of a prospective study of one hundred twenty patients. Thyroid 2000;10:709-712.

16 Renshaw AA. Follicular lesions of the thyroid. Am J Clin Pathol 2001;115:782-785.

17 Matesa N, Tabain I, Dabelic N, et al. Diagnostic relevance of fine needle aspiration cytology for follicular lesions of the thyroid: retrospective study. Croat Med J 2002;43:606-609.

18 Stelow EB, Bardales RH, Crary GS, et al. Interobserver variability in thyroid fine-needle aspiration interpretation of lesions showing predominantly colloid and follicular groups. Am J Clin Pathol 2005;124:239-244.

19 Deveci MS, Deveci G, Livolsi VA, et al. Fine-needle aspiration of follicular lesions of the thyroid. Diagnosis and follow-Up. Cytojournal 2006;3:9.

20 Mijovic T, Rochon L, Gologan O, et al. Fine-needle aspiration biopsies in the management of indeterminate follicular and Hurthle cell thyroid lesions. Otolaryngol Head Neck Surg 2009;140:715-719. 
21 Layfield LJ, Morton MJ, Cramer HM, et al. Implications of the proposed thyroid fine-needle aspiration category of 'follicular lesion of undetermined significance': A five-year multi-institutional analysis. Diagn Cytopathol 2009;37:710-714.

22 Nayar R, Ivanovic M. The indeterminate thyroid fineneedle aspiration: experience from an academic center using terminology similar to that proposed in the 2007 National Cancer Institute Thyroid Fine Needle Aspiration State of the Science Conference. Cancer 2009; 117:195-202.

23 Barden CB, Shister KW, Zhu B, et al. Classification of follicular thyroid tumors by molecular signature: results of gene profiling. Clin Cancer Res 2003;9: 1792-1800.

24 Aldred MA, Huang Y, Liyanarachchi S, et al. Papillary and follicular thyroid carcinomas show distinctly different microarray expression profiles and can be distinguished by a minimum of five genes. J Clin Oncol 2004;22:3531-3539.

25 Finley DJ, Zhu B, Barden CB, et al. Discrimination of benign and malignant thyroid nodules by molecular profiling. Ann Surg 2004;240:425-436.

26 Lubitz CC, Gallagher LA, Finley DJ, et al. Molecular analysis of minimally invasive follicular carcinomas by gene profiling. Surgery 2005;138:1042-1048.

27 Fryknas M, Wickenberg-Bolin U, Goransson $\mathrm{H}$, et al. Molecular markers for discrimination of benign and malignant follicular thyroid tumors. Tumour Biol 2006;27:211-220.

28 Pagedar NA, Chen DH, Wasman JK, et al. Molecular classification of thyroid nodules by cytology. Laryngoscope 2008;118:692-696.

29 Wiseman SM, Melck A, Masoudi H, et al. Molecular phenotyping of thyroid tumors identifies a marker panel for differentiated thyroid cancer diagnosis. Ann Surg Oncol 2008;15:2811-2826.

30 Griffith OL, Chiu CG, Gown AM, et al. Biomarker panel diagnosis of thyroid cancer: a critical review. Expert Rev Anticancer Ther 2008;8:1399-1413.

31 Cerutti JM, Latini FR, Nakabashi C, et al. Diagnosis of suspicious thyroid nodules using four protein biomarkers. Clin Cancer Res 2006;12:3311-3318.

32 Cerutti JM, Delcelo R, Amadei MJ, et al. A preoperative diagnostic test that distinguishes benign from malignant thyroid carcinoma based on gene expression. J Clin Invest 2004;113:1234-1242.

33 DeLellis RA, Lloyd RV, Heitz PU, et al. (eds). WHO Classification of Tumors. Pathology and Genetics.
Tumours of Endocrine Organs. IARC Press: Lyon, France, 2004.

34 DiVito KA, Charette LA, Rimm DL, et al. Long-term preservation of antigenicity on tissue microarrays. Lab Invest 2004;84:1071-1078.

35 Haataja L, Gurlo T, Huang CJ, et al. Many commercially available antibodies for detection of CHOP expression as a marker of endoplasmic reticulum stress fail specificity evaluation. Cell Biochem Biophys 2008; 51:105-107.

36 Matsumoto F, Fujii $\mathrm{H}$, Abe $\mathrm{M}$, et al. A novel tumor marker, Niban, is expressed in subsets of thyroid tumors and Hashimoto's thyroiditis. Hum Pathol 2006;37:1592-1600.

37 Patel MR, Stadler ME, Deal AM, et al. STT3A, C1orf24, TFF3: Putative Markers for Characterization of Follicular Thyroid Neoplasms From Fine-Needle Aspirates. Laryngoscope 2011;121:983-989.

38 Cerutti JM, Oler G, Delcelo R, et al. PVALB, a new Hurthle adenoma diagnostic marker identified through gene expression. J Clin Endocrinol Metab 2011;96: E151-E160.

39 Bryson PC, Shores CG, Hart C, et al. Immunohistochemical distinction of follicular thyroid adenomas and follicular carcinomas. Arch Otolaryngol Head Neck Surg 2008;134:581-586.

40 Netea-Maier RT, Hunsucker SW, Hoevenaars BM, et al. Discovery and validation of protein abundance differences between follicular thyroid neoplasms. Cancer Res 2008;68:1572-1580.

41 Hinsch N, Frank M, Doring C, et al. QPRT: a potential marker for follicular thyroid carcinoma including minimal invasive variant; a gene expression, RNA and immunohistochemical study. BMC Cancer 2009;9:93.

42 Griffith OL, Melck A, Jones SJ, et al. Meta-analysis and meta-review of thyroid cancer gene expression profiling studies identifies important diagnostic biomarkers. J Clin Oncol 2006;24:5043-5051.

43 Simon R, Radmacher MD, Dobbin K, et al. Pitfalls in the use of DNA microarray data for diagnostic and prognostic classification. J Natl Cancer Inst 2003;95: 14-18.

This work is licensed under the Creative Commons Attribution-NonCommercialNo Derivative Works 3.0 Unported License. To view a copy of this license, visit http://creativecommons. org/licenses/by-nc-nd/3.0/ 
